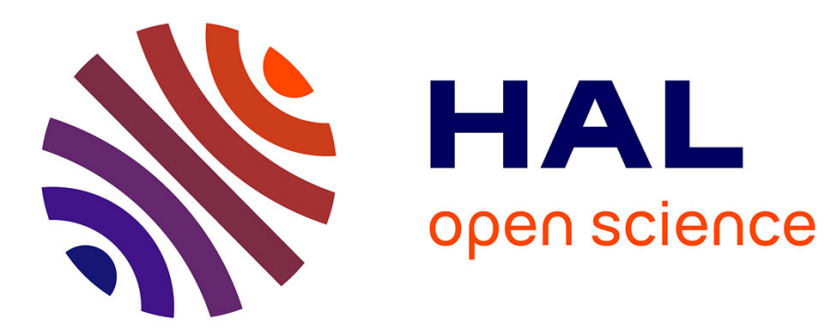

\title{
GROUPES ANALYTIQUES RIGIDES p-DIVISIBLES
}

Laurent Fargues

\section{To cite this version:}

Laurent Fargues. GROUPES ANALYTIQUES RIGIDES p-DIVISIBLES. Mathematische Annalen, 2019, 374 (1-2), pp.723-791. 10.1007/s00208-018-1782-9 . hal-03026670

\section{HAL Id: hal-03026670 https://hal.science/hal-03026670}

Submitted on 26 Nov 2020

HAL is a multi-disciplinary open access archive for the deposit and dissemination of scientific research documents, whether they are published or not. The documents may come from teaching and research institutions in France or abroad, or from public or private research centers.
L'archive ouverte pluridisciplinaire HAL, est destinée au dépôt et à la diffusion de documents scientifiques de niveau recherche, publiés ou non, émanant des établissements d'enseignement et de recherche français ou étrangers, des laboratoires publics ou privés. 


\title{
GROUPES ANALYTIQUES RIGIDES $p$-DIVISIBLES
}

\author{
LAURENT FARGUES
}

RÉSUMÉ. Nous définissons et étudions une catégorie de groupes analytiques rigides qui comprend les fibres génériques des groupes formels $p$-divisibles sur les anneaux d'entiers de corps $p$-adiques.

TABLE DES MATIÈRES

Introduction

1. Généralités sur les groupes analytiques rigides en caractéristique $0 \quad 5$

2. Les groupes analytiques rigides de type p-divisible 20

3. Classification des groupes analytiques rigides de type $p$-divisible sur un corps algébriquement clos

4. Classification des groupes analytiques rigides de type $p$-divisible sur un corps quelconque

5. Étude de la catégorie des groupes analytiques rigides de type $p$-divisible 36

6. Caractérisation géométrique des groupes formels $p$-divisibles 39

7. Une autre classification des $C$-groupes analytiques rigides de type $p$-divisible 41

8. Quasi-morphismes de groupes analytiques rigides de type $p$-divisible 43

Références $\quad 46$

\section{INTRODUCTION}

Soit $K$ un corps valué complet pour une valuation de rang 1 , extension de $\mathbb{Q}_{p}$. On note $\bar{K}$ une clôture algébrique de $K$ et $C=\widehat{\bar{K}}$.

Les espaces de Banach-Colmez. La motivation première de cet article concerne l'étude d'objets introduits par Pierre Colmez dans [9].

Dans [9] Colmez a défini des objets qu'il appelle espaces de Banach de dimension finie. Ce sont des foncteurs de la catégorie des « algèbres sympathiques »à valeurs dans les $\mathbb{Q}_{p}$-espaces de Banach. Les algèbres sympathiques sont des $C$-algèbres de Banach d'un type particulier.

Dans [13] nous développons un point de vue différent, plus géométrique, sur ces objets. Nous y définissons ce que nous appelons les espaces de Banach-Colmez effectifs comme étant certains espaces rigides généralisés en groupes de la forme

$$
\lim _{\overleftarrow{N}} G
$$

où $G$ est un groupe analytique rigide commutatif d'un type particulier que nous définissons et étudions dans cet article, «les groupes analytiques rigides de type $p$-divisible $\gg$, et les applications de transition sont la multiplication par $p$ sur $G$. Les $C$-points d'un tel espace rigide forment naturellement un $\mathbb{Q}_{p}$-espace de Banach. Nous définissons ensuite dans [13] les espaces de BanachColmez comme étant formellement les quotients d'un espace de Banach-Colmez effectif $X$ par un

Date: 11 novembre 2018.

2000 Mathematics Subject Classification. 14L05, 14G22.

Key words and phrases. p-divisible groups, rigid analytic geometry, p-adic Hodge theory.

The author acknowledges support from ANR-10-BLAN-0114 "ArShiFo". 
sous- $\mathbb{Q}_{p}$-espace vectoriel de dimension finie de l'espace de Banach $X(C)$. On montre alors par des méthodes différentes de celles de Colmez que la catégorie obtenue est abélienne. La catégorie obtenue est de plus équivalente à celle de [9] (bien que ce ne soit pas du tout évident).

Néanmoins, comme nous l'expliquons dans la suite de cette introduction, la définition et l'étude des groupes analytiques rigides de type $p$-divisible s'avère naturelle et intéressante en soi, indépendamment de l'article [13].

Fibre générique des groupes formels $p$-divisibles. Notons pdiv $\mathcal{O}_{K}$, resp. pdiv ${ }_{K}$, la catégorie des groupes $p$-divisibles $\operatorname{sur} \operatorname{Spec}\left(\mathcal{O}_{K}\right)$, resp. $\operatorname{Spec}(K)$. Il y a une équivalence de catégories

$$
\begin{aligned}
& \operatorname{pdiv}_{K} \stackrel{\sim}{\longrightarrow} \operatorname{Rep}_{\mathbb{Z}_{p}}(\operatorname{Gal}(\bar{K} \mid K)) \\
& H \longmapsto T_{p}(H)
\end{aligned}
$$

où $\operatorname{Rep}_{\mathbb{Z}_{p}}(\operatorname{Gal}(\bar{K} \mid K))$ désigne la catégorie des représentations continues du groupe de Galois à valeurs dans des $\mathbb{Z}_{p}$-modules libres de rang fini. Il y a de plus un foncteur fibre générique

$$
\begin{aligned}
\operatorname{pdiv}_{\mathcal{O}_{K}} & \longrightarrow \operatorname{pdiv}_{K} \\
H & \longmapsto H \otimes K
\end{aligned}
$$

Lorsque la valuation de $K$ est discrète Tate a montré ([17]) que ce foncteur est pleinement fidèle et que de plus, si $\operatorname{pdiv}\left(\mathcal{O}_{K}\right) \otimes \mathbb{Q}$ désigne la catégorie des groupes $p$-divisibles à isogénies près,

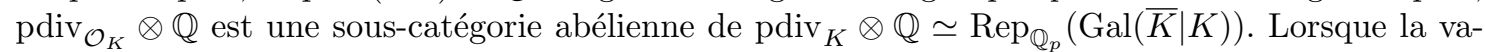
luation de $K$ n'est plus discrète, par exemple si $K$ est algébriquement clos, les résultats précédents ne sont pas vérifiés.

Prenons maintenant un point de vue différent. Il y a un foncteur de complétion formelle qui induit une équivalence de catégories

$$
\left\{\begin{array}{c}
\text { Groupes } p \text {-divisibles formels } \\
\operatorname{sur} \operatorname{Spec}\left(\mathcal{O}_{K}\right)
\end{array}\right\} \stackrel{\sim}{\longrightarrow}\left\{\begin{array}{c}
\text { Groupes formels } p \text {-divisibles } \\
\operatorname{sur} \operatorname{Spf}\left(\mathcal{O}_{K}\right)
\end{array}\right\} .
$$

Le membre de gauche désigne les groupes $p$-divisibles $H$ sur $\operatorname{Spec}\left(\mathcal{O}_{K}\right)$ tels que la réduction de $H[p]$ sur le corps résiduel de $K$ soit un groupe radiciel. Celui de droite désigne les groupes formels formellement lisses $\mathcal{H} \operatorname{sur} \operatorname{Spf}\left(\mathcal{O}_{K}\right)$ tels que la multiplication par $p$ sur $\mathcal{H}$ soit un morphisme fini localement libre. L'équivalence de catégories précédente est un analogue du théorème d'algébrisation de Grothendieck; l'équivalence entre les schémas abéliens polarisables et les schémas abéliens formels polarisables. Identifions les deux catégories précédentes et notons les pdiv ${ }_{\mathcal{O}_{K}}^{f}$. Prenant le point de vue des groupes formels il y a un foncteur fibre générique

$$
\begin{aligned}
\operatorname{pdiv}_{\mathcal{O}_{K}}^{f} & \longrightarrow K-\text { groupes formels commutatifs } \\
\mathcal{H} & \longmapsto \mathcal{H} \hat{\otimes} K
\end{aligned}
$$

Il y a cependant une équivalence de catégories

$$
\begin{aligned}
K \text {-groupes formels commutatifs } & \longrightarrow K-\text { e.v. de dimension finie } \\
\mathcal{G} & \longmapsto \text { Lie } \mathcal{G}
\end{aligned}
$$

d'inverse $W \longmapsto W \otimes \widehat{\mathbb{G}}_{a}$. Si $\mathcal{H}$ est un groupe formel $p$-divisible sa fibre générique au sens précédent, $\mathcal{H} \hat{\otimes} K$, ne détecte donc que l'algèbre de Lie, Lie $\mathcal{H}\left[\frac{1}{p}\right]$, c'est à dire le quotient de sa filtration de Hodge donné par les logarithmes de $\mathcal{H}$. Le foncteur fibré générique précédent n'est donc pas très intéressant puisqu'il fait perdre beaucoup d'informations sur le groupe formel $p$-divisible.

On se propose alors d'étudier un foncteur fibre générique plus subtil qui n'a pas été étudié auparavant, le foncteur

$$
\begin{aligned}
\operatorname{pdiv}_{\mathcal{O}_{K}}^{f} & \longrightarrow K-\text { groupes analytiques rigides commutatifs } \\
\mathcal{H} & \longmapsto \mathcal{H}^{r i g} .
\end{aligned}
$$

Via l'équivalence de catégories entre $K$-schémas localement de type fini de dimension 0 et $K$ espaces rigides de dimension 0 celui-ci permet de retrouver le foncteur fibre générique de Tate qui n'est rien d'autre que $\mathcal{H} \longmapsto \mathcal{H}^{\text {rig }}\left[p^{\infty}\right]$ ainsi que le foncteur fibre générique précédent via 
$\mathcal{H} \longmapsto$ Lie $\mathcal{H}^{\text {rig }}$. Il s'agit donc d'un foncteur fibre générique plus fin que les deux foncteurs présentés précédemment. Le théorème 6.1 de cet article s'énonce ainsi.

Théorème. Le foncteur fibre générique

$$
\begin{aligned}
\operatorname{pdiv}_{\mathcal{O}_{K}}^{f} & \longrightarrow K-\text { groupes analytiques rigides commutatifs } \\
\mathcal{H} & \longmapsto \mathcal{H}^{\text {rig }} .
\end{aligned}
$$

induit une équivalence de catégories entre la catégorie des groupes formels p-divisibles sur $\mathcal{O}_{K}$ et celle des $K$-groupes analytiques rigides commutatifs $G$ tels que

- comme espace analytique rigide $G \simeq \stackrel{\circ}{\mathbb{B}}^{d}(0,1)$ pour un entier $d$

- la multiplication par $p, G \stackrel{\times p}{\longrightarrow} G$, est un morphisme fini.

Ainsi, même si la valuation de $K$ n'est pas discrète, le foncteur fibre générique précédent est pleinement fidèle. Lorsqu'on travaille avec les groupes formels $p$-divisibles on peut oublier leurs modèles entiers et travailler directement au niveau des groupes analytiques rigides. Le corollaire 20 de cet article explique par exemple comment retrouver le module filtré associé à un groupe formel $p$-divisible directement à partir du groupe analytique rigide associé.

Les groupes analytiques rigides de type $p$-divisible. Si $H$ est un groupe $p$-divisible sur $\operatorname{Spec}(K)$, comme faisceau fppf $H$ est représentable par un $K$-schéma en groupes localement de type fini de dimension 0 . On vérifie alors que via le foncteur d'analytification la catégorie des groupes $p$-divisibles sur $K$ s'identifie à la catégorie des groupes analytiques rigides commutatifs $G$ tels que

- la multiplication par $p, G \stackrel{\times p}{\longrightarrow} G$ est un morphisme fini surjectif

- pour tout $x \in G, p^{n} x=0$ pour $n \gg 0$.

On se propose de relâcher la seconde condition en la remplaçant par une condition du type «topologiquement $p$-nilpotent $\gg$.

Définition. Un $K$-groupe analytique rigide de type $p$-divisible est un $K$-groupe analytique rigide commutatif $G$ tel que

- la multiplication par $p, G \stackrel{\times p}{\longrightarrow} G$, est un morphisme fini et surjectif

- si $\left|G^{a n}\right|$ désigne l'espace topologique sous-jacent à l'espace analytique de Berkovich associé $\grave{a} G$ alors pour tout $x \in\left|G^{a n}\right|, \lim _{n \rightarrow+\infty} p^{n} x=0$.

Notons $\mathcal{R}$ la catégorie des groupes analytiques rigides de type $p$-divisibles. Si $\mathcal{H}$ est un groupe formel $p$-divisible sur $\mathcal{O}_{K}$ il définit un groupe analytique rigide de type $p$-divisible $\mathcal{H}^{\text {rig }}$. Cela définit un foncteur pleinement fidèle

$$
\operatorname{pdiv}_{\mathcal{O}_{K}}^{f} \longrightarrow \mathcal{R}
$$

d'image essentielle les $G \in \mathcal{R}$ tels que $G \simeq \stackrel{\circ}{B}^{\operatorname{dim} G}(0,1)$. Par exemple si $A$ est une variété abélienne sur $K$ ayant bonne réduction soit

$$
G=\left\{x \in A^{a n} \mid \lim _{n \rightarrow+\infty} p^{n} x=0\right\}
$$

C'est un groupe analytique rigide de type $p$-divisible. De plus sa composante connexe neutre $G^{0}$ est la fibre générique du groupe formel $p$-divisible associé au modèle de Néron de $A$. Ainsi ce groupe formel (ou sa fibre générique) est entièrement déterminé par $A$ indépendamment du choix du modèle entier de $A$.

Si $H$ est un groupe $p$-divisible sur $K$ il définit alors un objet de $\mathcal{R}$ et cela définit un foncteur pleinement fidèle

$$
\operatorname{pdiv}_{K} \longrightarrow \mathcal{R}
$$

d'image essentielle les groupes analytiques rigides de type $p$-divisible de dimension 0 . Réciproquement, si $G \in \mathcal{R}$ alors $G\left[p^{\infty}\right] \in \operatorname{pdiv}_{K}$. Si $W$ est un $K$-espace vectoriel le groupe $W \otimes \mathbb{G}_{a}^{r i g}$, isomorphe à une somme de copies du groupe additif, est dans $\mathcal{R}$ et cela définit également un foncteur pleinement fidèle

$$
K-\text { e.v. de dimension finie } \longrightarrow \mathcal{R}
$$

La proposition 16 fournit le théorème de structure suivant. 
Proposition. Soit $G$ un groupe analytique rigide de type p-divisible. Il y a alors une suite exacte

$$
0 \longrightarrow G\left[p^{\infty}\right] \longrightarrow G \stackrel{\log }{\longrightarrow} \text { Lie } G \otimes \mathbb{G}_{a}^{\text {rig }} \longrightarrow 0
$$

où $G\left[p^{\infty}\right]$ est un groupe $p$-divisible, $\log$ est un morphisme étale surjectif qui fait de $G$ un revêtement étale de Lie $G \otimes \mathbb{G}_{a}^{\text {rig }}$ au sens de De Jong ([10]). Lorsque $G=\mathcal{H}^{\text {rig }}$ avec $\mathcal{H}$ un groupe formel $p$ divisible le morphisme log est donné par les logarithmes du groupe formel.

Ce théorème admet une réciproque, on renvoie à la proposition 18 .

Classification. L'un des résultats principaux de cet article est le théorème de classification suivant.

Théorème 0.1. La catégorie des $K$-groupes analytiques rigides de type p-divisible est équivalente à la catégorie des triplets $(\Lambda, W, f)$ où $\Lambda$ est une représentation continue de $G a l(\bar{K} \mid K)$ dans un $\mathbb{Z}_{p}$-module libre de type fini, $W$ est un $K$-espace vectoriel de dimension fini et

$$
f: W \otimes_{K} C \longrightarrow \Lambda \otimes_{\mathbb{Z}_{p}} C(-1)
$$

est un morphisme $C$-linéaire $G a l(\bar{K} \mid K)$-équivariant.

Si le groupe $G$ est associé au triplet $(\Lambda, W, f)$ on peut alors prendre $\Lambda=T_{p}\left(G\left[p^{\infty}\right]\right)$ et $W=$ Lie $G$. Lorsque $G=\mathcal{H}^{\text {rig }}$ avec $\mathcal{H}$ un groupe formel $p$-divisible sur $\mathcal{O}_{K}$ alors $f:$ Lie $\mathcal{H} \otimes C \longrightarrow T_{p}(\mathcal{H}) \otimes$ $C(-1)$ est la transposée de l'application de Hodge-Tate de $\mathcal{H}^{D}$.

Lorsque $K$ est algébriquement clos, c'est à dire $K=C$, le groupe analytique rigide de type $p$-divisible $G$ associé au triplet $(\Lambda, W, f)$ se construit par tiré en arrière via le diagramme suivant

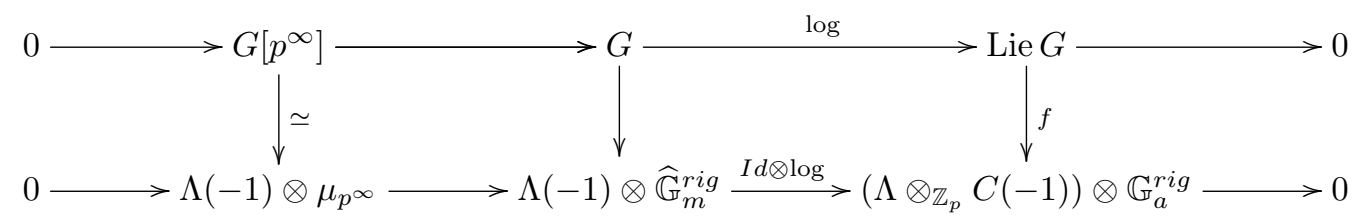

où $\widehat{\mathbb{G}}_{m}^{r i g}=\stackrel{\circ}{\mathbb{B}}(1,1)$ muni de sa structure multiplicative. Ainsi, après certains choix,

$$
G \simeq\left(\mathbb{G}_{a}^{r i g}\right)^{r} \oplus \log ^{-1}\left(V \otimes \mathbb{G}_{a}^{r i g}\right)
$$

où

$$
\log :\left(\widehat{\mathbb{G}}_{m}^{r i g}\right)^{s} \longrightarrow\left(\mathbb{G}_{a}^{r i g}\right)^{s}
$$

et $V \subset C^{s}$ est un sous- $C$-espace vectoriel. Le cas général se déduit du cas algébriquement clos par descente galoisienne.

Via la pleine fidélité du foncteur fibre générique on obtient le corollaire suivant valable pour tout $K$.

Corollaire. Soient $H_{1}$ et $H_{2}$ deux groupes p-divisibles formels sur $\mathcal{O}_{K}$. Alors

$$
\operatorname{Hom}\left(H_{1}, H_{2}\right)=\left\{u \in \operatorname{Hom}_{\mathbb{Z}_{p}[\operatorname{Gal}(\bar{K} \mid K)]}\left(T_{p}\left(H_{1}\right), T_{p}\left(H_{2}\right)\right) \mid u \otimes 1\left(\text { Lie H }_{1} \otimes C\right) \subset \text { Lie H }_{2} \otimes C\right\}
$$

Partant du corollaire précédent on obtient alors la généralisation suivante du théorème de Tate (théorème 5.1).

Théorème. Supposons $C(-1)^{\operatorname{Gal}(\bar{K} \mid K)}=0$. Alors, le foncteur fibre générique

est pleinement fidèle.

$$
\operatorname{pdiv}_{\mathcal{O}_{K}}^{f} \longrightarrow \operatorname{pdiv}_{K}
$$

Les théorèmes de classification suivants sont à la base des résultats de [13], ils sont le point de départ de la démonstration de l'analogue $\mathrm{du} \ll$ lemme fondamental $\gg$ de Colmez et Fontaine.

Théorème. Soit $G$ un $C$-groupe analytique rigide de type p-divisible connexe de dimension 1. Alors soit $G \simeq \mathbb{G}_{a}^{r i g}$ soit il existe un groupe formel p-divisible de dimension 1 sur $\mathcal{O}_{C}, H$, bien déterminé à isomorphisme unique près tel que $G \simeq H^{\text {rig }}$.

Théorème. Soit $G$ un $C$-groupe analytique rigide de type p-divisible. Il existe alors 
- des entiers $a, b \in \mathbb{N}$,

- des groupes formels p-divisibles de dimension $1, \mathcal{G}_{1}, \ldots, \mathcal{G}_{r}$ sur $\operatorname{Spf}\left(\mathcal{O}_{C}\right)$

- un sous-groupe p-divisible $\Gamma \subset \bigoplus_{i=1}^{r} \mathcal{G}_{i}^{\text {rig }}\left[p^{\infty}\right]$

tels que $G$ soit isomorphe au groupe

$$
G \simeq\left(\mathbb{Q}_{p} / \mathbb{Z}_{p}\right)^{a} \oplus\left(\mathbb{G}_{a}^{r i g}\right)^{b} \oplus\left(\bigoplus_{i=1}^{r} \mathcal{G}_{i}^{r i g}\right) / \Gamma .
$$

Structure de la catégorie des groupes analytiques rigides de type $p$-divisible à isogénie près. Il y a une bonne notion d'isogénie entre groupes analytiques rigides de type $p$-divisible. Notons $\mathcal{R} \otimes \mathbb{Q}$ pour la catégorie de tels groupes à isogénies près. Le théorème de classification précédent implique que cette catégorie est équivalente à celle des triplets $(V, W, f)$ où $V$ est un $\mathbb{Q}_{p}$-espace vectoriel de dimension finie muni d'une action linéaire continue de $\operatorname{Gal}(\bar{K} \mid K), W$ un $C$-espace vectoriel de dimension finie muni d'une action semi-linéaire continue de $\operatorname{Gal}(\bar{K} \mid K)$ et $f: W \longrightarrow V \otimes_{\mathbb{Q}_{p}} C(-1)$ est $C$-linéaire galois équivariant. De cela on déduit que la catégorie $\mathcal{R} \otimes \mathbb{Q}$ est abélienne. Via le foncteur fibre générique pleinement fidèle

$$
\operatorname{pdiv}_{\mathcal{O}_{K}}^{f} \otimes \mathbb{Q} \longrightarrow \mathcal{R} \otimes \mathbb{Q}
$$

on obtient donc un plongement de la catégorie des groupes $p$-divisibles formels à isogénie près (qui n'est pas abélienne en général si la valuation de $K$ n'est pas discrète) dans une catégorie abélienne.

La catégorie abélienne $\mathcal{R} \otimes \mathbb{Q}$ est munie de deux fonctions additives ht, $\operatorname{dim}: \mathcal{R} \otimes \mathbb{Q} \longrightarrow \mathbb{N}$ où ht $(G)$ désigne la hauteur du groupe $p$-divisible $G\left[p^{\infty}\right]$ et $\operatorname{dim}(G)$ la dimension de $G$. On définit une sous-catégorie exacte $\mathcal{R}^{a} \otimes \mathbb{Q}$ de $\mathcal{R} \otimes \mathbb{Q}$ formée des $G \in \mathcal{R}$ qui ne peuvent pas s'écrire sous la forme $\mathbb{G}_{a}^{r i g} \oplus G^{\prime}$ pour un $G^{\prime} \in \mathcal{R}$.

Proposition. Les objets de $\mathcal{R}^{a} \otimes \mathbb{Q}$ admettent des filtrations de type Harder-Narasimhan relativement à la fonction pente $\frac{\mathrm{dim}}{\mathrm{ht}}$.

La proposition précédente s'interpète en termes des résultats de [11]. On renvoie à la discussion suivant le théorème 5.2 .

Averstissement : Le premier chapitre de l'article concerne des généralités sur les groupes analytiques rigides malheureusement non disponibles dans la littérature. Il est inutile d'avoir lu l'intégralité de ce chapitre pour la compréhension de l'article, le lecteur pouvant s'y référer lorsqu'il en aura besoin.

\section{GÉNÉRAlités Sur LES GROUPES ANALYTIQUES RIGIDES EN CARACTÉRistiQUe 0}

1.1. Espaces rigides/espaces analytiques. Soit $K \mid \mathbb{Q}_{p}$ un corps valué complet pour une valuation de rang 1 . On fixe une clôture algébrique $\bar{K}$ de $K$ et on pose $C=\widehat{\bar{K}}$. On note $\left|K^{\times}\right|^{1 / \infty}=$ $\left|\bar{K}^{\times}\right|=\bigcup_{n>1}\left|K^{\times}\right|^{1 / n} \subset \mathbb{R}^{\times}$.

Nous faisons l'hypothèse suivante concernant les espaces analytiques au sens de Berkovich ([3], [4]) que nous considérerons.

Hypothèse : Les espaces analytiques au sens de Berkovich que nous considérerons seront tous des espaces analytiques stricts Hausdorff, c'est à dire dont l'espaces topologique sous-jacent est séparé.

Rappelons ([4], théorème 1.6.1) qu'il y a un foncteur pleinement fidèle de cette catégorie d'espaces analytiques vers les espaces rigides quasi-séparés. On notera $X^{\text {rig }}$ l'espace rigide associé à l'espace analytique $X$. Réciproquement si $Y$ est un espace rigide dans l'image essentielle du foncteur précédent on notera $Y^{a n}$ l'espace analytique associé. Rappelons également que les espaces rigides quasi-séparés possédant un recouvrement admissible affinoïde localement fini sont 
dans l'image essentielle de ce foncteur (ce qui représente la quasi-totalité des espaces rigides apparaissant dans la nature). Ils correspondent aux espaces analytiques dont l'espace topologique sous-jacent est paracompact. Il serait néanmoins maladroit de se restreindre aux seuls espaces analytiques paracompacts car un ouvert d'un tel espace peut ne pas être lui même paracompact.

Hypothèse : Dans toute la suite par espace rigide on entendra un espace rigide isomorphe à un espace $X^{\text {rig }}$ avec $X$ un espace analytique du type précédent.

Soit $X$ un espace rigide. Lorsqu'on écrira $x \in X$ cela signifiera que l'on considère un point « classique $\gg$ de $X$, ce qui du point de vue de Berkovich correspond aux $x \in X^{\text {an }}$ tels que $[\mathcal{K}(x): K]<+\infty$.

Dans tout ce qui suit la terminologie « rigide analytique »sera réservée aux espace rigides et $\ll$ analytique $\gg$ aux espaces analytiques au sens de Berkovich.

Rappelons que l'on a des notions de morphismes lisses et étales d'espaces rigides ([8], chapitre 2). Il y a également une notion de morphismes lisses et étales d'espaces analytiques au sens de Berkovich ([4], chapitre 3). Il s'agit d'une notion plus forte : via la correspondance $X \mapsto X^{a n}$ les morphismes lisses, resp. étales, d'espaces analytiques correspondent aux morphismes lisses, resp. étales, d'espaces rigides $X \rightarrow Y \ll$ surconvergents $\gg$ c'est à dire tels que $\partial\left(X^{a n} / Y^{a n}\right)=$ $\emptyset$. Les morphismes étales d'espaces rigides correspondent aux morphismes quasi-étales d'espaces analytiques tels que définis dans le chapitre 3 de [5]. Pour les morphismes étales finis les deux notions coïncident. On remarquera également que si $f: X \longrightarrow Y$ est un morphisme d'espaces rigides sur $K$ et $x \in X^{a n}$ est tel que $[\mathcal{K}(x): K]<+\infty$ alors $x \notin \partial(X / Y)$. En particulier si $f: X \longrightarrow Y$ est un morphisme étale d'espaces rigides alors $f^{a n}: X^{a n} \longrightarrow Y^{a n}$ est étale au voisinage de $x$.

Rappelons enfin ([4], théorème 3.4.1) que si $X$ est un espace analytique de Berkovich et $x \in X$ alors la catégorie des germes d'espaces analytiques étales sur des voisinages de $x$ dont la fibre en $x$ est finie s'identifie via l'application fibre en $x$ aux espaces analytiques étales finis sur $\mathcal{K}(x)$ c'est à dire aux espaces de la forme $\coprod_{i \in I} \mathcal{M}\left(L_{i}\right)$ avec $I$ fini et $L_{i} \mid \mathcal{K}(x)$ séparable finie. Ainsi si $f: X \longrightarrow Y$ est un morphisme étale d'espaces analytiques et $x \in X, f$ est un isomorphisme local au voisinage de $x$ si et seulement si $\mathcal{K}(x)=\mathcal{K}(f(x))$. En particulier si $f: X \longrightarrow Y$ est un morphisme étale d'espaces rigides et $x \in X, f^{a n}: X^{a n} \longrightarrow X^{a n}$ est un isomorphisme local au voisinage de $x$ si et seulement si $\mathcal{K}(x)=\mathcal{K}(f(x))$.

1.2. Généralités. Par définition un groupe analytique rigide sur $K$ est un groupe dans la catégorie des espaces rigides sur $K$. On notera $e$ la section unité ou parfois 0 lorsque le groupe est commutatif. On a de même une notion de groupe analytique dans la catégorie des espaces analytiques de Berkovich.

On remarquera que l'espace topologique $|G|$ associé au groupe analytique $G$ ne forme pas un groupe, il y a seulement un diagramme

$$
\begin{aligned}
& |G \times G| \stackrel{m}{\longrightarrow}|G| \\
& \downarrow \pi \\
& |G| \times|G|
\end{aligned}
$$

où l'application $\pi$ est propre et surjective. On remarquera néanmoins que les applications de translation par un point rationnel induisent une action de $G(K)$ par homéomorphismes sur $|G|$, $G(K) \longrightarrow \operatorname{Aut}(|G|)$. De plus l'inversion du groupe $G$ induit une involution $g \mapsto g^{-1}$ de l'espace topologique $|G|$. Plus généralement si $A$ et $\mathrm{B}$ sont deux sous-ensembles de $|G|$ posons $A \bullet \mathrm{B}=$ $m\left(\pi^{-1}(A \times \mathrm{B})\right)$. L'application $(A, \mathrm{~B}) \mapsto A \bullet \mathrm{B}$ satisfait les propriétés suivantes :

$-(A \bullet \mathrm{B}) \bullet C=A \bullet(\mathrm{B} \bullet C)$

- Si $A$ et B sont compacts $A \bullet$ B est compact, en particulier $\forall a, b \in G,\{a\} \bullet\{b\}$ est compact

— Si $a \in G(K)$ et $b \in G,\{a\} \bullet\{b\}=\{a . b\}$ où $b \mapsto a . b$ désigne l'action par translations de $a$ sur $|G|$ décrite précédemment 
- Utilisant le fait que $\pi$ est propre on vérifie que si $A$ est B sont compacts, si $W$ est un voisinage de $A \bullet \mathrm{B}$ il existe alors $U$ et $V$ des voisinages de $A$ et B tels que $U \bullet V \subset W$

- Du fait précédent on déduit en particulier que si $V$ est un voisinage de $e$ dans $|G|$ il existe alors un voisinage $U$ de $e$ dans $|G|$ tel que $U \bullet U \subset V$.

- On a $A \cap \mathrm{B} \neq \emptyset \Rightarrow e \in A \bullet \mathrm{B}^{-1}$

De plus les morphismes d'espaces analytiques $x \mapsto x^{n}$ de $G$ dans lui-même pour $n \in \mathbb{N}^{*}$ induisent une action du monoïde $\left(\mathbb{N}^{*}, \times\right)$ sur $|G|$. En particulier si $G$ est commutatif et $n \in \mathbb{N}$ il y a une application continue notée $g \mapsto n g$ de $|G|$ dans lui même.

Remarque 1. De la même façon que tout schéma en groupes sur un corps est séparé, tout groupe analytique rigide est automatiquement séparé puisque sa section unité est une immersion fermée (comme l'est tout point $K$-rationnel d'un espace rigide sur $K$ ). De même tout groupe analytique est séparé.

Remarque 2. Contrairement au cas de l'action d'un groupe de Lie par translations sur lui même, si $G$ est un groupe analytique l'action de $G(K)$ par translations sur $|G|$ n'est pas libre. Par exemple, si $G=(\mathbb{B}(0,1),+), x \in G$ désigne le point associé à la norme de Gauss, $\forall g \in G(K)=\mathcal{O}_{K}$, $g . x=x$. Plus généralement pour un $G$ quelconque $\partial(G / K)$ est un fermé de $|G|$ invariant sous l'action de $G(K)$ par translations. Ce fermé est un ensemble fini non vide si $G$ est affinö̈de.

Exemple 1. (1) Si $G$ est un groupe algébrique sur $K$ il définit un groupe analytique rigide $G^{\text {rig }}$.

(2) $S i V$ est un $K$-espace vectoriel de dimension finie on note $\mathbb{G}_{a}^{\text {rig }} \otimes V$ le groupe analytique rigide qui représente le foncteur $X \mapsto\left(\Gamma\left(X, \mathcal{O}_{X}\right) \otimes V,+\right)$. Après choix d'une base de $V$ il est isomorphe à une somme de copies de $\mathbb{G}_{a}^{\text {rig }}$. Cela définit un foncteur pleinement fidèle de la catégorie des $K$-espaces vectoriels de dimension finie dans celle des groupes analytiques rigides commutatifs.

(3) Si $\epsilon \in\left|K^{\times}\right|^{1 / \infty}$ et $d \geq 1$ est un entier, les boules ouvertes et fermées $\mathbb{B}^{d}(0, \epsilon)$ et $\mathbb{B}^{d}(0, \epsilon)$ sont des sous-groupes analytiques rigides ouverts de $\left(\mathbb{G}_{a}^{\text {rig }}\right)^{d}$.

(4) Si $\mathcal{G}$ est un groupe dans la catégorie des schémas formels quasi-séparés localement formellement de type fini sur $\operatorname{Sp} f\left(\mathcal{O}_{K}\right)$ il définit un groupe analytique rigide $\mathcal{G}^{\text {rig }}$.

(5) Le groupe analytique rigide $\mathbb{G}_{m}^{\text {rig }}$ représente le foncteur $X \mapsto \Gamma\left(X, \mathcal{O}_{X}\right)^{\times}$. Pour $\epsilon \in\left|K^{\times}\right|^{1 / \infty}$ avec $0<\epsilon \leq 1$ les foncteurs

$$
G_{\epsilon}: X \longmapsto\left\{f \in \Gamma\left(X, \mathcal{O}_{X}\right)^{\times}|\forall x \in X,| 1-f(x) \mid \leq \epsilon\right\}
$$

et

$$
\stackrel{\circ}{G}_{\epsilon}: X \longmapsto\left\{f \in \Gamma\left(X, \mathcal{O}_{X}\right)^{\times}|\forall x \in X,| 1-f(x) \mid<\epsilon\right\}
$$

sont représentés par des sous-groupes qui sont des ouverts admissibles de $\mathbb{G}_{m}^{\text {rig }}$. On a $G_{\epsilon}=$ $\mathbb{B}(1, \epsilon)$ et $\stackrel{\circ}{G}_{\epsilon}=\stackrel{\circ}{\mathbb{B}}(1, \epsilon)$. Pour $\epsilon=1$ on a $G_{1}=\widehat{\mathbb{G}}_{m}^{\text {rig }}$ où $\widehat{\mathbb{G}}_{m}$ désigne le complété p-adique de $\mathbb{G}_{m}$ sur $\operatorname{Spec}\left(\mathcal{O}_{K}\right)$. De plus $\stackrel{\circ}{G}_{1}=\widehat{\mathbb{G}}_{m}^{\text {rig }}$ où $\widehat{\mathbb{G}}_{m}$ désigne cette fois ci le complété formel de $\mathbb{G}_{m}$ sur $\operatorname{Spec}\left(\mathcal{O}_{K}\right)$ le long de sa section unité.

(6) L'exemple précédent s'étend au cas d'un tore algébrique $T$ sur $K$ en regardant $\{f \in T(X) \mid \forall \chi \in$ $\left.X^{*}(T), \forall x \in X,|1-\chi(f(x))| \leq \epsilon\right\}$ et de même avec < à la place de $\leq$. Lorsque $T$ est un tore non ramifié sur $K$ c'est à dire est la fibre générique d'un tore sur $\mathcal{O}_{K}$ et $\epsilon=1$ on a une description identique à la précédente comme fibre générique de schémas formels.

(7) $S i \mathcal{G}$ est un groupe formel formellement lisse de dimension d sur $\operatorname{Spf}\left(\mathcal{O}_{K}\right)$ la fibre générique $\mathcal{G}^{\text {rig }}$ est un groupe analytique rigide. Après choix d'un système de coordonnées formelles sur $\mathcal{G}, \mathcal{G}^{\text {rig }} \simeq \stackrel{\circ}{\mathbb{B}}^{d}(0,1)$ et cela définit donc une structure de groupe analytique rigide sur la boule ouvert de dimension $d$.

Étant donné que l'espace rigide $\mathcal{G}^{\text {rig }}$ est réduit il est déterminé par le foncteur $A \mapsto$ $\mathcal{G}^{\text {rig }}(A)$ définit sur les algèbres affinö̈des réduites. $\grave{A}$ une algèbre affinoïde réduite $A$ est associé canoniquement sa boule unité pour la norme sup $A^{0}=\left\{a \in A \mid\|a\|_{\infty} \leq 1\right\}$ qui est 
un réseau p-adiquement complet dans $A$ et définit donc un schéma formel p-adique $\operatorname{Sp} f\left(A^{0}\right)$. Alors

$$
\begin{aligned}
\mathcal{G}^{\text {rig }}: K-\text { alg. affinö̈des réduites } & \longrightarrow \text { Groupes } \\
A & \longmapsto \mathcal{G}\left(A^{0}\right) .
\end{aligned}
$$

Si $\epsilon \in\left|K^{\times}\right|^{1 / \infty}$ avec $\epsilon<1$, après un choix de coordonnées formelles sur $\mathcal{G}$, la boule ouverte, resp. fermée, de rayon $\epsilon$ forme un sous-groupe analytique rigide $\mathcal{G}_{\epsilon}^{\text {rig }}$, resp. $\mathcal{G}_{\epsilon}^{\text {rig }}$, de $\mathcal{G}^{\text {rig }}$. Ces sous-groupes sont indépendant du choix d'un système de coordonnées formelles sur $\mathcal{G}$. Pour $A$ affinoïde réduite notons $I_{\epsilon}=\left\{a \in A \mid\|a\|_{\infty} \leq \epsilon\right\}$, un idéal de $A^{0}$. Alors

$$
\mathcal{G}_{\epsilon}^{\text {rig }}(A)=\operatorname{ker}\left(\mathcal{G}\left(A^{0}\right) \longrightarrow \mathcal{G}\left(A^{0} / I_{\epsilon}\right)\right)
$$

et de même pour $\stackrel{\circ}{\mathcal{G}}_{\epsilon}$ en remplaçant $\leq$ par $<$. On a alors des recouvrements admissibles

$$
\mathcal{G}^{\text {rig }}=\bigcup_{\epsilon} \mathcal{G}_{\epsilon}^{\text {rig }}=\bigcup_{\epsilon} \dot{\mathcal{G}}_{\epsilon}^{\text {rig }} \text {. }
$$

(8) Soit $\Gamma$ un groupe muni d'une action discrète de $\operatorname{Gal}(\bar{K} \mid K), \operatorname{Gal}(\bar{K} \mid K) \rightarrow$ Aut $(\Gamma)$. Il définit un groupe analytique rigide étale $G$ tel que $\Gamma=G(\bar{K})$. Cela définit une équivalence de catégories entre la catégorie des groupes analytiques rigides étales sur $S p(K)$ et les groupes munis d'une action discrète de Gal $(\bar{K} \mid K)$. Par exemple tout groupe p-divisible $H$ sur $K$ définit un groupe analytique rigide étale. Le module galoisien associé est $T_{p}(H) \otimes \mathbb{Q}_{p} / \mathbb{Z}_{p}$. Les groupes p-divisibles sur $K$ correspondent aux modules Galoisiens discrets dont le groupe sous-jacent est $p$-divisible de cotype fini.

(9) Pour $\epsilon \in\left|K^{\times}\right|^{1 / \infty}$ soit $x_{\epsilon} \in \mathbb{G}_{a}^{a n}$ le point correspondant à la norme sup sur la boule fermée de rayon $\epsilon$. Alors $\left\{x_{\epsilon}\right\} \bullet\left\{x_{\epsilon^{\prime}}\right\}$ est égal à $\left\{x_{\sup \left\{\epsilon, \epsilon^{\prime}\right\}}\right\}$ si $\epsilon \neq \epsilon^{\prime}$ et $\mathbb{B}(0, \epsilon)$ si $\epsilon=\epsilon^{\prime}$.

Soit $G$ un groupe analytique rigide. Définissons le foncteur

$\widehat{G}:$ K-algèbres affinoïdes $\longrightarrow$ groupes

$$
A \longmapsto\left\{s \in G(A) \mid \exists I \text { idéal nilpotent de } A, s_{\mid A / I}=e \in G(A / I)\right\} .
$$

On a $\widehat{G} \subset G$, un sous faisceau en groupes sur le gros site rigide de $\operatorname{Sp}(K)$. Il s'agit du complété formel de $G$ le long de sa section unité. La catégorie des $K$-algèbres artiniennes locales de corps résiduel $K$ se plonge dans la catégorie des $K$-algèbres affinoïdes. On considère maintenant $\widehat{G}$ comme foncteur sur de telles algèbres artiniennes. Si $\mathcal{I} \subset \mathcal{O}_{G}$ désigne l'idéal cohérent définissant la section unité de $G$ alors pour tout $k \geq 0$ le faisceau d'algèbres $\mathcal{O}_{G} / \mathcal{I}^{k+1}$ provient par image réciproque de $\operatorname{Sp}(K)$ d'un $K$-espace vectoriel de dimension finie,

$$
\Gamma\left(G, \mathcal{O}_{G} / \mathcal{I}^{k+1}\right),
$$

module que l'on notera de façon abusive $\mathcal{O}_{G} / \mathcal{I}^{k+1}$. Alors

$$
\widehat{G} \simeq \operatorname{Spf}\left(\lim _{k \geq 0} \mathcal{O}_{G} / \mathcal{I}^{k+1}\right)
$$

qui est donc un groupe formel au sens de [14]. Puisque $K$ est de caractéristique 0 d'après le corollaire 3.3.1 de [14], $\widehat{G}$ est formellement lisse ; le choix d'un relèvement d'une base de $\mathcal{I} / \mathcal{I}^{2}$ dans la restriction de $\mathcal{I}$ à un ouvert affinö̈de contenant la section unité induit un isomorphisme

$$
K \llbracket X_{1}, \ldots, X_{d} \rrbracket \stackrel{\sim}{\longrightarrow} \lim _{k \geq 0} \mathcal{O}_{G} / \mathcal{I}^{k+1}
$$

où $d=\operatorname{dim} \mathcal{I} / \mathcal{I}^{2}$. On note

$$
\begin{aligned}
\omega_{G} & =\mathcal{I} / \mathcal{I}^{2} \\
\operatorname{Lie} G & =\omega_{G}^{*} .
\end{aligned}
$$

Proposition 1. Tout groupe analytique rigide sur $K$ est lisse. Il existe de plus un ouvert affinoüde $U$ contenant la section unité et $\epsilon \in\left|K^{\times}\right|^{1 / \infty}$ tels que $U \simeq \mathbb{B}^{d}(0, \epsilon)$ où $d=\operatorname{dim} \omega_{G}$, la section unité s'envoyant sur l'origine de la boule. 
Démonstration. Soit $e$ la section unité de $G$. L'anneau local noethérien $\mathcal{O}_{G, e}$ est tel que $\widehat{\mathcal{O}}_{G, e} \simeq$ $\lim \mathcal{O}_{G} / \mathcal{I}^{k+1} \simeq K \llbracket X_{1}, \ldots, X_{d} \rrbracket$. L'anneau $\mathcal{O}_{G, e}$ est donc régulier. D'après le lemme 2.8 de $[8]$ on $\overleftarrow{k \geq 0}$

en déduit que $G$ est lisse au voisinage de $e$. Puisque le corps résiduel en $e$ coïncide avec le corps de base l'assertion concernant l'existence d'un $U$ tel que dans l'énoncé en résulte. Maintenant si $x \in G$ soit $\mathcal{K}(x)$ le corps résiduel de $x$. C'est une extension de degré fini de $K$. Puisque le morphisme $\mathrm{Sp}(\mathcal{K}(x)) \rightarrow \operatorname{Sp}(K)$ est étale, pour montrer que $G$ est lisse au voisinage de $x$ il suffit de montrer que $G \otimes_{K} \mathcal{K}(x)$ est lisse sur $\mathcal{K}(x)$ au voisinage d'un point s'envoyant sur $x$ via $G \otimes \mathcal{K}(x) \rightarrow G$. Mais il existe un tel point $\mathcal{K}(x)$-rationnel. Par translation on est donc ramené au cas de la section unité.

Du point de vue des espaces analytiques de Berkovich, la proposition précédente implique qu'il existe un voisinage ouvert de l'élément neutre de $G^{a n}$ isomorphe à une boule ouverte de dimension $d$ et rayon $\epsilon$ avec $\epsilon \in\left|K^{\times}\right|^{1 / \infty}$ (un $K$-point d'un $K$-espace analytique de Berkovich est contenu dans l'intérieur de tout domaine analytique le contenant, en particulier avec les notations de l'énoncé $e$ est dans l'intérieur de $U^{a n}$ ).

Corollaire 1. Un groupe analytique $G$ sur $K$ est lisse si et seulement si $\partial(G / K)=\emptyset$.

Corollaire 2. Il y a des équivalences entre les catégories suivantes :

- Les groupes analytiques rigides étales sur $K$.

- Les groupes analytiques rigides sur $K$ de dimension 0.

- Les groupes analytiques étales sur $K$.

- Les groupes analytiques sur $K$ de dimension 0.

- Les groupes « abstraits $\gg$ munis d'une action discrète de Gal $(\bar{K} \mid K)$ par automorphismes de groupes.

Si $q: G \longrightarrow \operatorname{Sp}(K)$ désigne le morphisme structural il y a une équivalence de catégories

Faisceaux cohérents $G$-équivariants sur $G \stackrel{\sim}{\longrightarrow} K-$ e.v. de dimension finie

$$
\begin{aligned}
\mathcal{F} & \longmapsto e^{*} \mathcal{F} \\
q^{*} \mathcal{G} & \longleftarrow \mathcal{G}
\end{aligned}
$$

où par faisceau $G$-équivariant on entend une action compatible avec l'action de $G$ par translations à gauche sur lui même. Via cette équivalence on obtient

$$
\Omega_{G}^{1} \simeq q^{*} \omega_{G} .
$$

Considérons l'espace des formes différentielles invariantes par translation à gauche, resp. à droite, sur $G$. Il s'agit des $\alpha \in \Gamma\left(G, \Omega_{G}^{1}\right)$ telles que pour tout $K$-espace rigide $S$ et toute section $s$ : $S \longrightarrow G_{S}$ du $S$-espace rigide $G_{S} \longrightarrow S$ si $h: G_{S} \longrightarrow G$ est la projection et $t_{s}: G_{S} \stackrel{\sim}{\longrightarrow} G_{S}$ la translation à gauche, resp. à droite, par la section $s$ on ait $t_{S}^{*}\left(h^{*} \alpha\right)=h^{*} \alpha \in \Gamma\left(G_{S}, \Omega_{G_{S} / S}^{1}\right)$. Soit $m: G \times G \longrightarrow G$ la multiplication. On a $\Omega_{G \times G / K}^{1}=\operatorname{pr}_{1}^{*} \Omega_{G / K}^{1} \oplus \operatorname{pr}_{2}^{*} \Omega_{G / K}^{1}$. Alors le $K$-espace vectoriel des formes invariantes à gauche s'identifie à

$$
\left\{\alpha \in \Gamma\left(G, \Omega_{G / K}^{1}\right) \mid m^{*} \alpha \equiv \operatorname{pr}_{1}^{*} \alpha \bmod \operatorname{pr}_{2}^{*} \Omega_{G / K}^{1}\right\}
$$

resp. $m^{*} \alpha \equiv \operatorname{pr}_{2}^{*} \alpha \bmod \operatorname{pr}_{1}^{*} \Omega_{G / K}^{1}$ pour les formes invariantes à droite. Lorsque $G$ est commutatif c'est encore

$$
\left\{\alpha \in \Gamma\left(G, \Omega_{G / K}^{1}\right) \mid m^{*} \alpha=\operatorname{pr}_{1}^{*} \alpha+\operatorname{pr}_{2}^{*} \alpha\right\} .
$$

L'application fibre en l'origine induit un isomorphisme

$$
\{\text { formes invariantes à gauche }\} \stackrel{\sim}{\sim} \omega_{G}
$$

et de même pour les formes invariantes à droite. L'action par translation à droite sur les formes invariantes à gauche induit un morphisme de groupes rigides

$$
G \longrightarrow \mathrm{GL}\left(\omega_{G}\right)^{r i g}
$$


qui est la transposée de l'action adjointe sur l'algèbre de Lie. Cela signifie que si $S$ est un espace rigide le morphisme associé $\rho: G(S) \longrightarrow \operatorname{Aut}_{\Gamma\left(S, \mathcal{O}_{S}\right)}\left(\Gamma\left(S, \mathcal{O}_{S}\right) \otimes_{K} \omega_{G}\right)$ est défini de la façon suivante : pour $x \in G(S)$ soit $\operatorname{int}_{x}: G_{S} \stackrel{\sim}{\longrightarrow} G_{S}$ l'automorphisme du $S$-espace rigide en groupes $G_{S}$ qui est la conjugaison par la section définie par $x$, alors $\rho(x)=$ int $_{x}^{*}: \omega_{G_{S} / S} \stackrel{\sim}{\longrightarrow} \omega_{G_{S} / S}$ avec $\omega_{G_{S} / S}=\mathcal{O}_{S} \otimes \omega_{G}$.

Puisque tout morphisme d'une variété rigide propre vers l'espace rigide $X^{\text {rig }}$ associé à une variété algébrique $X$ se factorise via un sous-espace de $X^{\text {rig }}$ fini sur $K$ on déduit la proposition qui suit.

Proposition 2. Tout groupe analytique rigide connexe et propre sur $K$ est commutatif.

La correspondance $G \mapsto$ Lie $G$ définit un foncteur de la catégorie des groupes analytiques rigides sur $K$ vers les $K$-algèbres de Lie de dimension finie.

Lemme 1. Soit $f: G_{1} \longrightarrow G_{2}$ un morphisme de groupes analytiques rigides. Soit Lie $(f)$ : Lie $G_{1} \longrightarrow$ Lie $G_{2}$. Alors,

- $f$ est lisse si et seulement si Lie $(f)$ est surjective.

- $f$ est étale si et seulement si Lie $(f)$ est bijective.

- $f$ est non-ramifié si et seulement si Lie $(f)$ est injective.

Démonstration. D'après la proposition 2.6 de [8] cela résulte de ce que $G_{1}$ et $G_{2}$ sont lisses et des formules pour $i \in\{1,2\}$, si $q_{i}: G_{i} \longrightarrow \operatorname{Sp}(K), \Omega_{G_{i} / K}=q_{i}^{*} \omega_{G_{i}}$ et $\omega_{G_{i}}=e^{*} \Omega_{G_{i} / K}$.

\subsection{Structure des morphismes étales.}

1.3.1. Structure. Puisque le corps résiduel en l'élément neutre d'un groupe analytique est $K$ on a le lemme qui suit.

Lemme 2. Un morphisme $f: G_{1} \longrightarrow G_{2}$ de groupes analytiques rigides est étale si et seulement si $f^{a n}: G_{1}^{a n} \longrightarrow G_{2}^{a n}$ est un isomorphisme au voisinage de l'élément neutre.

Le lemme qui suit dit que pour étudier les morphismes étales de groupes analytiques on peut se ramener au cas surjectif.

Lemme 3. Soit $f: G_{1} \longrightarrow G_{2}$ un morphisme étale de groupes analytiques. Alors $f\left(G_{1}\right)$ est un sous-groupe ouvert de $G_{2}$.

Démonstration. Puisque $f$ est étale $f\left(G_{1}\right)$ est un ouvert $U$ de $G_{2}$ ([4], proposition 3.2.7). Il suffit de voir que le morphisme induit par la multiplication de $G_{2}, U \times U \longrightarrow G_{2}$, est à valeurs dans $U$. Mais cela résulte de la surjectivité de l'application $\left|G_{1} \times G_{1}\right| \longrightarrow|U \times U|$.

Si $f: G_{1} \longrightarrow G_{2}$ est un morphisme étale de groupes analytiques rigides alors ker $f$ est un sous-groupe étale Zariski fermé dans $G_{1}$. On a la caractérisation suivante de tels groupes.

Lemme 4. Soit $G$ un groupe analytique sur $K$. On note $G(\bar{K})=\bigcup_{\substack{L \mid K \text { finie } \\ L \subset \bar{K}}} G(L)$. Les ensembles suivants sont en bijection :

(1) Les sous-groupes Zariski fermés de $G$ de dimension 0.

(2) Les sous-groupes Zariski fermés de $G^{\text {rig }}$ de dimension 0.

(3) Les sous-groupes $\operatorname{Gal}(\bar{K} \mid K)$-stables $\Gamma$ de $G(\bar{K})$ discrets dans le $\widehat{\bar{K}}$-espace analytique naïf $G(\widehat{\bar{K}})$ tels que $\Gamma$ soit fermé dans $|G \hat{\otimes} \widehat{\bar{K}}|$

(4) Les sous-groupes $G a l(\bar{K} \mid K)$-stables $\Gamma$ de $G(\bar{K})$ tels que pour tout domaine affinoüde $V$ de $G, V(\widehat{\bar{K}}) \cap \Gamma$ soit fini.

Exemple 2. Soit $G$ un groupe analytique rigide commutatif et $n \geq 1$ un entier. Puisque la multiplication par $n$ sur l'algèbre de Lie de $G$ est un isomorphisme, $\times n: G \longrightarrow G$ est étale. Le sous-groupe fermé $G[n]$ des points de $n$-torsion de $G$ est donc un sous-groupe étale Zariski fermé de $G$. 
Remarque 3. Si $H$ est un groupe topologique séparé et $\Gamma$ un sous-groupe discret il est alors automatiquement fermé. Dans le cadre des espaces analytiques cela n'est pas à priori vrai en général; pour un groupe analytique $G$ sur $K$ et un sous-groupe discret $\Gamma \subset G(K)$, $\Gamma$ est fermé dans le groupe topologique $G(K)$ mais pas forcément dans l'espace topologique $|G|$ (c'est tout de même vrai si $K$ est localement compact).

Lemme 5. Soit $f: G_{1} \longrightarrow G_{2}$ un morphisme étale surjectif de groupes analytiques. Sont équivalents :

(1) $f$ est fini

(2) ker $f$ est fini sur $K$ i.e. le noyau du morphisme de groupes abstraits $G_{1}(\bar{K}) \longrightarrow G_{2}(\bar{K})$ est fini

Démonstration. On a clairement $(1) \Rightarrow(2)$. Supposons (2) vérifiée. Quitte à faire un changement de base et remplacer $K$ par une extension de degré fini on peut supposer que ker $f$ est un « groupe abstrait $\gg$, un sous-groupe fini de $G(K)$. Soit $W$ un sous-ensemble compact de $\left|G_{2}\right|$. Puisque $f$ est une application ouverte surjective il existe un compact $V$ de $\left|G_{1}\right|$ tel que $W \subset f(V)$. Alors $f^{-1}(W) \subset \bigcup_{\gamma \in \operatorname{ker} f} \gamma . V$ qui est donc compact. L'application $|f|:\left|G_{1}\right| \longrightarrow\left|G_{2}\right|$ est donc propre. Puisque $f$ est étale $\partial\left(G_{1} / G_{2}\right)=\emptyset$ et donc $f$ est un morphisme propre d'espaces analytiques. Étant de plus quasi-fini on en déduit qu'il est fini.

Lemme 6. Soit $G$ un groupe analytique et $\Gamma \subset G(K)$ un sous-groupe discret fermé dans $|G|$. Alors l'action de $\Gamma$ sur $|G|$ est proprement discontinue. En particulier le stabilisateur d'un élément de $|G|$ est un sous-groupe fini de $\Gamma$.

Démonstration. Si $V$ est un domaine analytique compact dans $G$ et $\gamma \in \Gamma$ on a $\gamma \cdot V \cap V \neq \emptyset \Rightarrow$ $\gamma \in V^{-1} \bullet V$. Or $V^{-1} \bullet V$ est compact dans $|G|$.

Exemple 3. Si $X$ est un espace analytique et $\Gamma$ est un groupe agissant de façon proprement discontinue et sans points fixes sur $|X|$ on peut former l'espace analytique quotient $\Gamma \backslash X$, l'application de projection $X \longrightarrow \Gamma \backslash X$ étant un isomorphisme local.

Soit maintenant $G$ un groupe analytique commutatif et $\Gamma \subset G(K)$ un sous-groupe discret sans torsion fermé dans $|G|$. Alors l'action de $\Gamma$ sur $|G|$ est proprement discontinue sans point fixe et on peut alors former l'espace analytique quotient $G / \Gamma$ qui est un groupe analytique commutatif. Le morphisme $G \longrightarrow G / \Gamma$ est un morphisme de groupes qui est un isomorphisme local au voisinage de tout point de $G$ de noyau le groupe analytique étale associé à $\Gamma$. Si $\partial(G / K)=\emptyset$ et $|G| / \Gamma$ est compact alors $G / \Gamma$ est un espace analytique propre sur $K$.

Rappelons ([10]) qu'un morphisme $f: X \longrightarrow Y$ d'espaces analytiques est un revêtement étale si tout point de $Y$ possède un voisinage ouvert $U$ tel que l'on ait $f^{-1}(U)=\coprod_{i \in I} V_{i}$ avec pour tout $i, V_{i} \longrightarrow U$ étale fini. Cette catégorie de revêtements contient à la fois les morphismes étales finis ainsi que les revêtements de l'espace topologique sous-jacent à l'espace analytique.

Lemme 7. Un morphisme d'espaces analytiques $f: X \longrightarrow Y$ est un revêtement étale si et seulement si le morphisme $X \hat{\otimes} C \longrightarrow Y \hat{\otimes} C$ en est un.

Démonstration. La projection $|Y \hat{\otimes} C| \longrightarrow|Y|$ est une application surjective ouverte. On en déduit que si le morphisme $X \hat{\otimes} C \longrightarrow Y \hat{\otimes} C$ est un revêtement étale tout point de $Y$ possède un voisinage $U$ tel que $f^{-1}(U) \hat{\otimes} C=\coprod_{i \in I} V_{i}$ avec pour tout $i, V_{i} \longrightarrow U \hat{\otimes} C$ étale fini. Soit $W$ une composante connexe de $f^{-1}(U)$. On a donc $W \hat{\otimes} C=\coprod_{j \in J} T_{j}$ avec $T_{j} \longrightarrow W \hat{\otimes} C$ étale fini. Mais $\pi_{0}(W \hat{\otimes} C)$ est fini et toute composante connexe de $W \hat{\otimes} C$ est définie sur une extension de degré fini de $K$. On en déduit que $W$ est finie sur $U$.

Proposition 3. Soit $f: G_{1} \longrightarrow G_{2}$ un morphisme étale surjectif de groupes analytiques. Alors $f$ est un revêtement étale. Supposons de plus $K$ algébriquement clos et soit $\Gamma=\operatorname{ker} f$. Pour tout $x \in G_{1}$ soit $\Gamma_{x}$ le stabilisateur de $x$ dans $\Gamma$. Alors $\mathcal{K}(x) \mid \mathcal{K}(f(x))$ est une extension galoisienne finie, $\Gamma_{x} \stackrel{\sim}{\longrightarrow} \operatorname{Gal}(\mathcal{K}(x) \mid \mathcal{K}(f(x)))$ et il existe un voisinage ouvert $V$ de $x$ tel que

$$
f^{-1}(f(V))=\coprod_{\bar{\gamma} \in \Gamma / \Gamma_{x}} \gamma \cdot V
$$


avec $f_{\mid \gamma . V}: \gamma . V \longrightarrow f(V)$ étale fini galoisien de groupe $\Gamma_{x}$.

Démonstration. D'après le lemme 7 on peut supposer que $K$ est algébriquement clos. Soit $\Gamma \subset G_{1}(K)$ le noyau de $f$. Soit $x \in G_{2}$. Choisissons $y \in G_{1}$ tel que $f(y)=x$. Puisque $f$ est étale il existe des voisinages $U$ de $x$ et $V$ de $y$ tels que $f_{\mid V}: V \longrightarrow U$ soit étale fini surjectif. Quitte à rétrécir $V$ et $U$ on peut de plus supposer que $V$ est relativement compact dans $\left|G_{1}\right|$. Alors d'après le lemme $6\{\gamma \in \Gamma \mid \gamma \cdot V \cap V\}$ est fini. Quitte à rétrécir $V$ et $U$ on peut donc supposer que pour $\gamma \in \Gamma, \gamma \cdot V \cap V \neq \emptyset \Rightarrow \gamma \cdot y=y$. Notons $\Gamma_{y}$ le stabilisateur de $y$. Alors

$$
f^{-1}(U)=\coprod_{\bar{\gamma} \in \Gamma / \Gamma_{y}} \gamma \cdot V
$$

avec $\gamma \cdot V \longrightarrow V$ étale fini. De plus la fibre en $x$ de $f_{\mid V}, V_{x} \longrightarrow \mathcal{M}(\mathcal{K}(x))$ est un espace analytique étale fini tel que $V_{x} \times_{\mathcal{M}(\mathcal{K}(x))} V_{x} \simeq \coprod_{\Gamma_{y}} V_{x}$ (par définition du noyau de $f, G_{1} \times_{G_{2}} G_{1} \simeq G_{1} \times$ ker $f$, tirant cette égalité en arrière via $\mathcal{M}(\mathcal{K}(x)) \longrightarrow G_{1}$ on obtient le résultat). On en déduit le résultat.

Exemple 4. Supposons $K$ algébriquement clos. Soit log : $\widehat{\mathbb{G}}_{m}^{a n} \longrightarrow \mathbb{G}_{a}^{a n}$ le logarithme où $\widehat{\mathbb{G}}_{m}^{a n}=$ $\left\{x \in\left(\mathbb{A}^{1}\right)^{a n}|| 1-x \mid<1\right\}$. C'est un morphisme étale et ker $\log =\mu_{p}(K)$. En restriction à la boule ouvert $\stackrel{\circ}{\mathbb{B}}\left(1, p^{-\frac{1}{p-1}}\right)$ c'est un isomorphisme sur son image. Maintenant si $\epsilon \in\left[p^{-\frac{1}{p-1}}, 1\left[\cap\left|K^{\times}\right|^{1 / \infty}\right.\right.$ soit le sous-groupe affinoïde $\mathbb{B}(1, \epsilon) \subset \mathbb{G}_{m}^{a n}$. Soit

$$
r_{\epsilon}=\sup \left\{r \geq 1 \mid p^{-\frac{1}{p^{r-1}(p-1)}} \leq \epsilon\right\} .
$$

Alors le morphisme

$$
\log _{\mid \mathbb{B}(1, \epsilon)}: \mathbb{B}(1, \epsilon) \longrightarrow \log (\mathbb{B}(1, \epsilon))=\mathbb{B}(0, \psi(\epsilon))
$$

est un morphisme étale fini galoisien de groupe $\mu_{p^{r_{\epsilon}}}$ où la fonction $\epsilon \mapsto \psi(\epsilon)$ se calcule explicitement. De plus

$$
\log ^{-1}(\log (\mathbb{B}(1, \epsilon)))=\coprod_{\bar{\zeta} \in \mu_{p} \infty(K) / \mu_{p^{r}}(K)} \mathbb{B}\left(\zeta, r_{\epsilon}\right) .
$$

Si $\epsilon \in] 0,1\left[\cap\left|K^{\times}\right|^{1 / \infty}\right.$ et $x_{\epsilon} \in \widehat{\mathbb{G}}_{m}^{a n}$ est le point correspondant à la norme de Gauss associée à la boule $\mathbb{B}(1, \epsilon)$ alors si $\epsilon<p^{-\frac{1}{p-1}}, \mathcal{K}\left(x_{\epsilon}\right)=\mathcal{K}\left(\log x_{\epsilon}\right)$ et pour $p^{-\frac{1}{p^{r-1}(p-1)}} \leq \epsilon<p^{-\frac{1}{p^{r}(p-1)}}$ avec $r \geq 1$

$$
\mu_{p^{r}}(K) \stackrel{\sim}{\longrightarrow} \operatorname{Gal}\left(\mathcal{K}\left(x_{\epsilon}\right) \mid \mathcal{K}\left(\log x_{\epsilon}\right)\right)
$$

Par contraste, log est un isomorphisme local au voisinage de tout point classique i.e. tout élément de $\widehat{\mathbb{G}}_{m}^{a n}(K)$.

Remarque 4. Contrairement au cas des groupes de Lie, si $G$ est un groupe analytique en général $\pi_{1}(G, e)$ n'est pas commutatif (ici on considère la groupe fondamental étendu au sens de De Jong ([10])). Par exemple la tour de Lubin-Tate fournit un quotient du groupe fondamental de $\left(\stackrel{\circ}{B}^{1}(0,1),+\right)$ isomorphe à $G L_{2}\left(\mathbb{Z}_{p}\right)$. De même, contrairement au cas des groupes de Lie, si $G$ est un groupe analytique, $E \longrightarrow G$ est un revêtement étale et que l'on a un relèvement à $E$ de la section unité de $G$, il n'existe pas en général de structure de groupe analytique sur $E$ ayant pour section neutre la section choisie et telle que $E \longrightarrow G$ soit un morphisme de groupes.

1.3.2. Construction de morphismes étales. On a vu dans l'exemple 3 comment construire des cas particuliers de morphismes étales de groupes analytiques commutatifs. Néanmoins en général les morphismes étales sont plus généraux que ceux donnés dans l'exemple 3.

Rappelons qu'un bon espace analytique est un espace analytique pour lequel tout point possède un voisinage affinoïde.

Théorème 1.1. Soit $X$ un bon espace analytique. Soit $\Gamma$ un groupe agissant sur $X$ tel que l'action induite de $\Gamma$ sur $|X|$ soit proprement discontinue. Supposons que pour tout $x \in X$ le morphisme de groupes $\operatorname{Stab}_{\Gamma}(x) \longrightarrow \operatorname{Aut}(\mathcal{K}(x))$ soit injectif. Alors le faisceaux étale $\Gamma \backslash X$ est représentable par un bon espace analytique. Le morphisme $X \longrightarrow \Gamma \backslash X$ est un revêtement étale au sens de De Jong [10] et induit un homéomorphisme $\Gamma \backslash|X| \stackrel{\sim}{\longrightarrow}|\Gamma \backslash X|$.

Commençons par démontrer un cas particulier du théorème précédent. 
Proposition 4. Soit $A$ une $K$-algèbre affinoïde et $\Gamma$ un groupe fini d'automorphismes de $A$ qui induit une action de $\Gamma$ sur $\mathcal{M}(A)$. Supposons que $\forall x \in \mathcal{M}(A)$ le morphisme $\operatorname{Stab}_{\Gamma}(x) \longrightarrow A u t(\mathcal{K}(x))$ soit injectif. Alors le faisceau étale $\Gamma \backslash \mathcal{M}(A)$ est représenté par $\mathcal{M}\left(A^{\Gamma}\right)$, le morphisme $\mathcal{M}(A) \longrightarrow$ $\Gamma \backslash \mathcal{M}(A)$ est étale fini galoisien de groupe $\Gamma$ et $\Gamma \backslash|\mathcal{M}(A)| \stackrel{\sim}{\longrightarrow}|\Gamma \backslash \mathcal{M}(A)|$.

Démonstration. D'après la proposition $6.3 .3 / 3$ de [6] $A^{\Gamma}$ est bien une algèbre affinoïde (ce qui était sous-entendu dans l'énoncé).

Soit $\mathfrak{p} \in \operatorname{Spec}(A)$ et $\Gamma_{\mathfrak{p}}$ le stabilisateur de $\mathfrak{p}$. Montrons que l'application $\Gamma_{\mathfrak{p}} \longrightarrow \operatorname{Aut}(k(\mathfrak{p}))$ est injective. Pour cela considérons l'application surjective « support d'une valuation »

$$
\operatorname{supp}: \mathcal{M}(A) \longrightarrow \operatorname{Spec}(A) \text {. }
$$

Choisissons $x \in \mathcal{M}(A)$ tel que $\operatorname{supp}(x)=\mathfrak{p}$. Soit $\gamma \in \Gamma_{\mathfrak{p}} \backslash\{e\}$. Si $\gamma(x) \neq x$ il est alors clair que $\gamma$ agit non trivialement $\operatorname{sur} A / \mathfrak{p}$ et donc $\operatorname{sur} k(\mathfrak{p})=\operatorname{Frac}(A / \mathfrak{p})$. Si $\gamma \cdot x=x$, la valuation $x$ définit une valuation sur $k(\mathfrak{p})$ et pour cette valuation $\mathcal{K}(x)=\widehat{k(\mathfrak{p})}$. Puisque $\gamma$ agit continûment sur $k(\mathfrak{p})$ pour la topologie définie par $x$ et que $\gamma$ agit non trivialement sur son complété, $\gamma$ agit non trivialement sur $k(\mathfrak{p})$.

D'après le corollaire $2.3 \mathrm{du}$ chapitre $\mathrm{V}$ de [1] le morphisme $\operatorname{Spec}(A) \longrightarrow \operatorname{Spec}\left(A^{\Gamma}\right)$ est un revêtement étale galoisien de groupe $\Gamma$. Donc, $A \hat{\otimes}_{A^{\Gamma}} A=A \otimes_{A^{\Gamma}} A \simeq \prod_{\Gamma} A$ et le morphisme $\mathcal{M}(A) \longrightarrow \mathcal{M}\left(A^{\Gamma}\right)$ est étale fini galoisien de groupe $\Gamma$.

L'application surjective $\pi:|\mathcal{M}(A)| \longrightarrow\left|\mathcal{M}\left(A^{\Gamma}\right)\right|$ est clairement invariante sous $\Gamma$ et induit donc une application continue surjective $\Gamma \backslash|\mathcal{M}(A)| \longrightarrow\left|\mathcal{M}\left(A^{\Gamma}\right)\right|$. Par compacité de $\Gamma \backslash|\mathcal{M}(A)|$ pour montrer que c'est un homéomorphisme il suffit de montrer qu'elle est injective. Cela se déduit de la surjectivité de l'application composée

$$
\coprod_{\Gamma}|\mathcal{M}(A)| \simeq\left|\mathcal{M}(A) \times_{\mathcal{M}\left(A^{\Gamma}\right)} \mathcal{M}(A)\right| \longrightarrow|\mathcal{M}(A)| \times_{\left|\mathcal{M}\left(A^{\Gamma}\right)\right|}|\mathcal{M}(A)| .
$$

Démonstration du théorème 1.1. Soit $x \in X$ On note $\Gamma_{x}$ le stabilisateur de $x$ dans $\Gamma$, un groupe fini. Puisque l'action de $\Gamma$ sur $|X|$ est proprement discontinue et que $x$ possède un voisinage affinoïde il existe un voisinage affinoïde $V_{x}$ de $x$, tel que $\gamma \cdot V_{x} \cap V_{x} \neq \emptyset \Rightarrow \gamma \in \Gamma_{x}$. D'après la proposition 4 on peut former le quotient $V_{x} \longrightarrow \Gamma_{x} \backslash V_{x}$. Si $\dot{V}_{x}$ désigne l'intérieur de $V_{x}$ dans $X$ on s'intéressera plutôt au quotient

$$
\pi_{x}: \stackrel{\circ}{V}_{x} \longrightarrow \Gamma_{x} \backslash \stackrel{\circ}{V}_{x}
$$

On applique maintenant le a) de la proposition 1.3.3 de [4] afin de recoller les $\Gamma_{x} \backslash \stackrel{\circ}{V}_{x}$ lorsque $x$ parcourt $X$. Il suffit pour cela de définir pour tous $x, y \in X$ un isomorphisme canonique

$$
\pi_{x}\left(\stackrel{\circ}{V}_{x} \cap \stackrel{\circ}{V}_{y}\right) \stackrel{\sim}{\longrightarrow} \pi_{y}\left(\stackrel{\circ}{V}_{x} \cap \stackrel{\circ}{V}_{y}\right)
$$

Mais comme faisceau étale ces deux espaces s'identifient canoniquement à

$$
\Gamma_{x} \cap \Gamma_{y} \backslash \stackrel{\circ}{V}_{x} \cap \stackrel{\circ}{V}_{y} .
$$

Corollaire 3. Soit $G$ un groupe analytique commutatif dont l'espace analytique sous-jacent est bon et $\Gamma \subset G(K)$ un sous-groupe discret fermé dans $|G|$. On peut alors former le groupe analytique quotient $G / \Gamma$ et le morphisme surjectif $G \longrightarrow G / \Gamma$ est un revêtement étale.

Remarque 5. Le lecteur n'aura aucune difficulté à étendre le théorème 1.1 au cas d'un groupe étale $\Gamma$ sur $\mathcal{M}(K)$ agissant sur un bon espace analytique $X$ tel l'action de $\Gamma(\bar{K})$ sur $|X \hat{\otimes} C|$ satisfasse aux hypothèses du théorème 1.1. Le corollaire 3 s'étend de même.

Remarque 6. L'auteur ne sait pas étendre le théorème 1.1 lorsque l'espace analytique n'est plus bon (ce qui est une hypothèse assez restrictive). Néanmoins un tel résultat peut être étendu aux cas des espaces adiques localement de type fini quasi-séparés sur Spa $(K)$.

\subsection{Le groupe des composantes connexes d'un groupe analytique.}


1.4.1. Sur les composantes connexes des espaces analytiques. Rappelons que si $X$ est un espace analytique l'espace topologique $|X|$ est localement connexe par arcs et donc les composantes connexes de $X$ sont ouvertes. On en déduit que $X$ est union disjointe de ses composantes connexes. Si $X=\mathcal{M}(A)$ est affinoïde alors $\pi_{0}(X)=\pi_{0}(\operatorname{Spec}(A))$.

Lemme 8. Soit $X$ un espace analytique et $\mathcal{C}$ un ensemble de domaines affinoïdes de $X$ tel que tout point de $X$ possède un voisinage formé d'une union finie d'éléments de $\mathcal{C}$. Alors

$$
\operatorname{coker}\left(\coprod_{V, V^{\prime} \in \mathcal{C}} \pi_{0}\left(V \cap V^{\prime}\right) \longrightarrow \coprod_{V \in \mathcal{C}} \pi_{0}(V)\right) \stackrel{\sim}{\longrightarrow} \pi_{0}(X)
$$

Proposition 5. Supposons $K$ algébriquement clos. Soient $X$ et $Y$ deux espaces analytiques sur K. Alors l'application

$$
\pi_{0}(X \times Y) \longrightarrow \pi_{0}(X) \times \pi_{0}(Y)
$$

est une bijection.

Démonstration. On peut supposer que $X$ et $Y$ sont réduits, ce qui est alors le cas de $X \times Y$. On utilise les notations et les rappels de la section 1.7. Si $A$ est une $K$-algèbre affinoïde réduite on a des identifications

$$
\pi_{0}(\mathcal{M}(A))=\pi_{0}\left(\operatorname{Spec}\left(A^{0}\right)\right)=\pi_{0}(\operatorname{Spec}(\widetilde{A})) .
$$

De plus si $A$ et $B$ sont réduites

$$
(A \hat{\otimes} \mathrm{B})^{\sim}=\widetilde{A} \otimes \widetilde{\mathrm{B}} .
$$

Donc, puisque le corps résiduel de $K$ est algébriquement clos

$$
\begin{aligned}
\pi_{0}(\mathcal{M}(A) \times \mathcal{M}(\mathrm{B})) & =\pi_{0}(\mathcal{M}(A \hat{\otimes} \mathrm{B}))=\pi_{0}\left(\operatorname{Spec}\left((A \hat{\otimes} \mathrm{B})^{\sim}\right)\right)=\pi_{0}(\operatorname{Spec}(\widetilde{A}) \times \operatorname{Spec}(\widetilde{\mathrm{B}})) \\
& =\pi_{0}(\operatorname{Spec}(\widetilde{A})) \times \pi_{0}(\operatorname{Spec}(\widetilde{\mathrm{B}}))=\pi_{0}(\mathcal{M}(A)) \times \pi_{0}(\mathcal{M}(\mathrm{B})) .
\end{aligned}
$$

D'où le résultat si $X$ et $Y$ sont affinoïdes. Maintenant si $X$ et $Y$ sont quelconques soit $\mathcal{C}, \operatorname{resp} . \mathcal{D}$, l'ensemble des domaines affinö̈des dans $X$, resp. $Y$. D'après le lemme 8

$$
\pi_{0}(X \times Y)=\operatorname{coker}\left(\coprod_{\substack{V, V^{\prime} \in \mathcal{C} \\ W, W^{\prime} \in \mathcal{D}}} \pi_{0}(\underbrace{(V \times W) \times\left(V^{\prime} \times W^{\prime}\right)}_{V \cap V^{\prime} \times W \cap W^{\prime}}) \longrightarrow \coprod_{\substack{V \in \mathcal{C} \\ W \in \mathcal{D}}} \pi_{0}(V \times W)\right) .
$$

Si $X$ et $Y$ sont séparés alors $\forall V, V^{\prime} \in \mathcal{C}, V \cap V^{\prime} \in \mathcal{C}$ et $\forall W, W^{\prime} \in \mathcal{D}, W \cap W^{\prime} \in \mathcal{D}$. Dans ce cas là on a donc d'après le cas affinoïde et une application du lemme 8 à $X$ et $Y$

$$
\begin{aligned}
\pi_{0}(X \times Y) & =\operatorname{coker}\left(\coprod_{\substack{V, V^{\prime} \in \mathcal{C} \\
W, W^{\prime} \in \mathcal{D}}} \pi_{0}\left(V \cap V^{\prime}\right) \times \pi_{0}\left(W \cap W^{\prime}\right) \longrightarrow \coprod_{\substack{V \in \mathcal{C} \\
W \in \mathcal{D}}} \pi_{0}(V) \times \pi_{0}(W)\right) \\
& \stackrel{\sim}{\longrightarrow} \pi_{0}(X) \times \pi_{0}(Y) .
\end{aligned}
$$

Maintenant pour $V, V^{\prime} \in \mathcal{C}$ et $W, W^{\prime} \in \mathcal{D}, V \cap V^{\prime}$ et $W \cap W^{\prime}$ sont séparés. On peut donc appliquer le cas précédent pour obtenir $\pi_{0}\left(V \cap V^{\prime} \times W \cap W^{\prime}\right) \stackrel{\sim}{\longrightarrow} \pi_{0}\left(V \cap V^{\prime}\right) \times \pi_{0}\left(W \cap W^{\prime}\right)$. Par une nouvelle application du calcul précédent on obtient le résultat pour $X$ et $Y$ généraux.

Proposition 6. Soit $X$ un $K$-espace analytique.

(1) Sont équivalents :

- $X \hat{\otimes} C$ est connexe

- Pour toute extension de degré fini $L \mid K, X \otimes L$ est connexe

Si $X$ est connexe et possède un point $K$-rationnel alors les conditions équivalentes précédentes sont vérifiées.

(2) Toute composante connexe de $X \hat{\otimes} C$ est définie sur une extension de degré fini de K. L'action de $\operatorname{Gal}(\bar{K} \mid K)$ sur $\pi_{0}(X \hat{\otimes} C)$ est discrète. Il y a une bijection

$$
\operatorname{Gal}(\bar{K} \mid K) \backslash \pi_{0}(X \hat{\otimes} C) \stackrel{\sim}{\longrightarrow} \pi_{0}(X) .
$$

En particulier si $X$ est connexe, $\pi_{0}(X \hat{\otimes} C)$ est fini. 
Démonstration. Pour toutes les assertions on se ramène facilement au cas où $X$ est connexe, ce que l'on suppose donc. La projection $|X \hat{\otimes} C| \longrightarrow|X|$ induit un homéomorphisme

$$
\operatorname{Gal}(\bar{K} \mid K) \backslash|X \hat{\otimes} C| \stackrel{\sim}{\longrightarrow}|X| \text {. }
$$

En particulier, la projection $\pi:|X \hat{\otimes} C| \longrightarrow|X|$ est une application continue ouverte et propre. Cela implique que l'image par $\pi$ d'un fermé est un fermé (toute application continue et propre entre espaces topologiques dont le but est localement compact envoie un fermé sur un fermé). On en déduit que si $U$ est une composante connexe de $X \hat{\otimes} C$ alors $\pi(U)=X$. Soit donc $U$ une telle composante connexe et $x \in U(C)$ tel que $[\mathcal{K}(\pi(x)): K]<+\infty$. Alors $\forall \sigma \in \operatorname{Gal}(\bar{K} \mid \mathcal{K}(\pi(x))), \sigma . x=$ $x$ et donc puisque $\sigma . U$ est une composante connexe de $X \hat{\otimes} C$ telle que $U \cap \sigma . U \neq \emptyset$ on a $U=\sigma . U$. L'action de $\operatorname{Gal}(\bar{K} \mid K)$ sur $\pi_{0}(X \hat{\otimes} C)$ est donc discrète. De plus si $V$ est une autre composante connexe, puisque $\pi^{-1}\{\pi(x)\} \neq \emptyset, \exists y \in V, \exists \sigma \in \operatorname{Gal}(\bar{K} \mid K), \sigma . y=x$ et donc $\sigma . V=U$. De cela on déduit que $\operatorname{Gal}(\bar{K} \mid K) \backslash \pi_{0}(X \hat{\otimes} C) \stackrel{\sim}{\longrightarrow} \pi_{0}(X)$. Du raisonnement précédent on déduit également que si $X$ possède un point $K$-rationnel alors $X \hat{\otimes} C$ est connexe.

Toutes les autres assertions s'en déduisent facilement car après une extension de degré fini $X$ possède un point rationnel.

Définition 1. Soit $X$ un $K$-espace analytique. On note $\underline{\pi}_{0}(X)$ le-K-espace analytique étale tel que $\underline{\pi}_{0}(X)(C)=\pi_{0}(X \hat{\otimes} C)$ comme $G a l(\bar{K} \mid K)$-module discret.

Des résultats précédents on déduit :

Proposition 7. Il existe un morphisme $X \longrightarrow \underline{\pi}_{0}(X)$ surjectif qui est universel parmi les morphisme de $X$ vers des $K$-espace analytiques étales. Il induit de plus une bijection $G a l(\bar{K} \mid K)$ équivariante $\pi_{0}(X \hat{\otimes} C) \stackrel{\sim}{\longrightarrow} \underline{\pi}_{0}(X)(C)$.

Pour deux $K$-espaces analytiques $X$ et $Y$ il y a un isomorphisme naturel

$$
\underline{\pi}_{0}(X \times Y) \stackrel{\sim}{\longrightarrow} \underline{\pi}_{0}(X) \times \underline{\pi}_{0}(Y) .
$$

Une autre façon de décrire $\underline{\pi}_{0}(X)$ est de dire qu'il représente le faisceau étale $f_{*} \mathcal{F}$ où $\mathcal{F}$ est l'objet final du topos $\tilde{X}_{\text {ét }}$ (le faisceau constant sur l'ensemble à un élément) et $f: X \longrightarrow \mathcal{M}(K)$.

1.4.2. Composantes connexes des groupes analytiques. Soit $G$ un groupe analytique sur $K$. On note $G^{0}$ la composante connexe de la section unité $e$.

Lemme 9. $G^{0}$ est un sous-groupe analytique ouvert distingué de $G$.

Démonstration. Puisque $G^{0}$ est connexe et possède un point rationnel, d'après les résultats de la section précédente $G^{0} \times G^{0}$ est connexe. Donc, la loi de groupe de $G$ restreint à $G^{0}, G^{0} \times G^{0} \longrightarrow G$, se factorise par $G^{0}$. Soit $\varphi: G \times G^{0} \longrightarrow G$ le morphisme $(x, y) \mapsto x y x^{-1}$. Si $X$ est une composante connexe de $G$ alors $X \times G^{0}$ est connexe (une fois de plus car $G^{0}$ possède un point rationnel) et est donc une composante connexe de $G \times G^{0}$. Mais $e \in \pi\left(X \times G^{0}\right)$ et donc $\pi\left(X \times G^{0}\right) \subset G^{0}$. Donc $G^{0} \triangleleft G$.

Puisque pour deux espaces analytiques $X$ et $Y, \underline{\pi}_{0}(X \times Y) \stackrel{\sim}{\longrightarrow} \underline{\pi}_{0}(X) \times \underline{\pi}_{0}(Y)$, le foncteur $\underline{\pi}_{0}$ transforme des groupes analytiques sur $K$ en des groupes analytiques étales sur $K$. On obtient donc un groupe analytique étale $\underline{\pi}_{0}(G)$. On démontre alors aisément la proposition qui suit.

Proposition 8. Il y a une suite exacte de faisceaux sur le grand site étale de $\mathcal{M}(K)$

$$
1 \longrightarrow G^{0} \longrightarrow G \longrightarrow \underline{\pi}_{0}(G) \longrightarrow 1
$$

qui fait des composantes connexes de $G$ des $G^{0}$-torseurs triviaux au dessus des composantes connexes de $\underline{\pi}_{0}(G)$ triviaux après une extension de degré fini.

1.5. Analyticité du logarithme sur un voisinage de l'élément neutre. Hypothèse : désormais tous nos groupes analytiques rigides seront commutatifs

Le choix d'une base de $\omega_{G}$ induit un isomorphisme entre le groupe formel $\widehat{G}$ et $\widehat{\mathbb{G}}_{a}^{d}$. De façon plus intrinsèque $\widehat{G} \stackrel{\sim}{\longrightarrow} \widehat{\mathbb{G}}_{a} \otimes$ Lie $G$. Fixons une base de $\omega_{G}$ et soit $\left(\omega_{1}, \ldots, \omega_{d}\right) \in \Gamma\left(G, \Omega_{G}^{1}\right)$ la base correspondante des formes différentielles invariantes par translation. Elles induisent une 
base $\left(\hat{\omega}_{1}, \ldots, \hat{\omega}_{d}\right) \in\left(\widehat{\Omega}_{\widehat{G}}^{1}\right)^{d}$ des formes invariantes par translations sur le groupe formel $\widehat{G}$. On a $\omega_{G}=\mathcal{I} / \mathcal{I}^{2}$ et dans un ouvert affinoïde $U$ tel que dans la proposition 1 on peut relever cette base d'éléments de $\omega_{G}$ en des section de $\mathcal{I}$ telle manière que ces relevés induisent un isomorphisme

$$
U \stackrel{\sim}{\longrightarrow} \mathbb{B}^{d}(0, \epsilon)
$$

pour un $\epsilon \in\left|K^{\times}\right|^{1 / \infty}$. Par transport on obtient donc des formes $\omega_{1}, \ldots, \omega_{d} \in \Gamma\left(\mathbb{B}^{d}(0, \epsilon), \Omega_{\mathbb{B}^{d}(0, \epsilon)}^{1}\right)$ telles que

$$
\omega_{i}=\sum_{j} f_{i j} d X_{j}
$$

avec $f_{i j} \in \Gamma\left(\mathbb{B}^{d}(0, \epsilon), \mathcal{O}_{\mathbb{B}^{d}(0, \epsilon)}\right), f_{i j}(0, \ldots, 0)=0$ et $f_{i j} \equiv 0[\operatorname{deg} \geq 2]$ si $i \neq j$ et $f_{i i} \equiv X_{i}[\operatorname{deg} \geq 2]$.

Il existe un unique isomorphisme de groupes formels

$$
h: \widehat{G} \stackrel{\sim}{\longrightarrow} \widehat{\mathbb{G}}_{a}^{d}
$$

tel que $\left(h^{*} d T_{1}, \ldots, h^{*} d T_{d}\right)=\left(\hat{\omega}_{1}, \ldots, \hat{\omega}_{d}\right)$. Dans les coordonnées données par l'isomorphisme $U \stackrel{\sim}{\longrightarrow}$ $\mathbb{B}^{d}(0, \epsilon)$ cet isomorphisme est donné par un isomorphisme

$$
\begin{aligned}
K \llbracket T_{1}, \ldots, T_{d} \rrbracket & \stackrel{\sim}{\longrightarrow} K \llbracket X_{1}, \ldots, X_{d} \rrbracket \\
T_{i} & \longmapsto h_{i}
\end{aligned}
$$

tel que

$$
\frac{\partial h_{i}}{\partial X_{j}}=f_{i j}
$$

En d'autres termes le logarithme du groupe formel est donné par intégration des formes différentielles invariantes. De cela on déduit aisément que $\forall \epsilon^{\prime}<\epsilon$ tel que $\epsilon^{\prime} \in\left|K^{\times}\right|^{1 / \infty}$ et tout $i$

$$
h_{i} \in \Gamma\left(\mathbb{B}^{d}\left(0, \epsilon^{\prime}\right), \mathcal{O}_{\mathbb{B}^{d}\left(0, \epsilon^{\prime}\right)}\right)
$$

et que donc quitte à prendre $\epsilon$ plus petit le logarithme $h$ s'étend en un isomorphisme $U \stackrel{\sim}{\longrightarrow} \mathbb{B}^{d}(0, \epsilon)$.

Proposition 9. Il existe $\epsilon \in\left|K^{\times}\right|^{1 / \infty}$, un sous-groupe affinoïde $U$ de $G$ et un isomorphisme

$$
\log : U \stackrel{\sim}{\longrightarrow}\left(\mathbb{B}(0, \epsilon)^{d},+\right)
$$

associé au choix d'une base de Lie $G$ qui induit le logarithme du groupe formel $\widehat{G}$ après complétion formelle de $U$ le long de sa section unité et de $\mathbb{B}(0, \epsilon)^{d}$ le long de l'origine.

Démonstration. On a déjà vu l'existence de $\log : U \stackrel{\sim}{\longrightarrow} \mathbb{B}(0, \epsilon)^{d}$ pour un $\epsilon$ suffisamment petit. Reste à voir que quitte à prendre $\epsilon^{\prime} \leq \epsilon$, remplacer $\epsilon$ par $\epsilon^{\prime}$ et $U$ par $\log ^{-1}\left(\mathbb{B}\left(0, \epsilon^{\prime}\right)^{d}\right), U$ est un sous-groupe et log est un morphisme de groupes. Le tiré en arrière de la loi de groupe additive sur $\mathbb{B}(0, \epsilon)^{d}$ définit un morphisme $U \times U \longrightarrow U$ et donc $m_{1}: U \times U \longrightarrow G$. La loi de groupe de $G$ définit $m_{2}: U \times U \longrightarrow G$. On sait que $m_{1}$ et $m_{2}$ induisent les mêmes morphismes de $K$-schémas formels $\widehat{U} \times \widehat{U} \longrightarrow \widehat{G}$. Considérons la différence $f=m_{1} \cdot m_{2}^{-1}: U \times U \longrightarrow G$. Soit $Y=f^{-1}(e) \subset U \times U$, un sous-espace rigide Zariski fermé de $U \times U$ tel que $(e, e) \in Y$. De plus le sous-schéma formel fermé de $\widehat{U} \times \widehat{U}$ induit par $Y \subset U \times U$ coïncide avec $\widehat{U} \times \widehat{U}$. Soit $\mathcal{I}$ l'idéal cohérent définissant $Y$ dans $U \times U$. Soit $\mathcal{I}_{(e, e)}$ sa fibre en $(e, e) \in U \times U$, un $\mathcal{O}_{U \times U,(e, e)}$-module de type fini. L'anneau local $\mathcal{O}_{U \times U,(e, e)}$ est noethérien et de plus $\widehat{U} \times \widehat{U}=\operatorname{Spf}\left(\widehat{\mathcal{O}}_{U \times U,(e, e)}\right)$. Étant donné que $\mathcal{O}_{U \times U,(e, e)} \longrightarrow \widehat{\mathcal{O}}_{U \times U,(e, e)}$ est fidèlement plat on en déduit que $\mathcal{I}_{(e, e)}=\mathcal{O}_{U \times U,(e, e)}$. Mais $\mathcal{I}_{(e, e)}=\underset{\epsilon^{\prime} \rightarrow 0}{\lim } \mathcal{I}\left(\log ^{-1}\left(\mathbb{B}^{d}\left(0, \epsilon^{\prime}\right)\right)\right)$. Donc quitte à prendre $\epsilon$ plus petit on peut supposer que $Y=U$.

Corollaire 4. Tout groupe analytique rigide commutatif de dimension d possède un ouvert admissible affinoïde qui est un sous-groupe analytique rigide isomorphe à $\left(\mathbb{B}^{d}(0, \epsilon),+\right)$ pour $\epsilon \in\left|K^{\times}\right|^{1 / \infty}$.

Tout groupe analytique commutatif de dimension $d$ au sens de Berkovich possède une base de voisinages de 0 formée de sous-groupes analytiques ouverts isomorphes à $\left(\mathbb{B}^{d}(0, \epsilon),+\right)$ pour $\epsilon \in\left|K^{\times}\right|^{1 / \infty}$.

Remarque 7. Si $K$ est algébriquement clos, les groupes $\left(\mathbb{B}^{d}(0, \epsilon),+\right)$, resp. $\left(\mathbb{B}^{d}(0, \epsilon),+\right)$, avec $\epsilon \in\left|K^{\times}\right|^{1 / \infty}$ sont isomorphes et on peut prendre $\epsilon=1$. 
1.6. Un théorème de structure des groupes analytiques rigides commutatifs.

Théorème 1.2. Soit $G$ un $K$-groupe analytique commutatif. Soit

$$
U=\left\{g \in|G| \mid \lim _{n \rightarrow+\infty} p^{n} g=0\right\}
$$

Alors $U$ est un sous-groupe analytique ouvert de $G$. On a $G\left[p^{\infty}\right] \subset U$, un sous-groupe étale tel que $\left|G^{a n}\left[p^{\infty}\right]\right|$ soit fermé et discret dans $U$. Il existe un unique morphisme de groupes analytiques

$$
\log : U \longrightarrow \mathbb{G}_{a}^{a n} \otimes \text { Lie } G
$$

induisant l'identité sur les algèbres de Lie. C'est un isomorphisme au voisinage de l'élément neutre de G. De plus la suite de groupes

$$
0 \longrightarrow G\left[p^{\infty}\right] \longrightarrow U \stackrel{\log }{\longrightarrow} \mathbb{G}_{a}^{a n} \otimes \operatorname{Lie} G
$$

est exacte pour la topologie étale et le morphisme $\log ^{\text {rig }}: U^{\text {rig }} \longrightarrow \mathbb{G}_{a}^{\text {rig }} \otimes$ Lie $G$ est étale.

Démonstration. Soit $V$ un voisinage de 0 dans $G^{a n}$ qui forme un sous-groupe analytique et $\epsilon \in\left|K^{\times}\right|^{1 / \infty}$ tels qu'il y ait un isomorphisme

$$
\log : V \stackrel{\sim}{\longrightarrow}\left(\mathbb{B}^{d}(0, \epsilon),+\right)
$$

induisant le logarithme de $\widehat{G}$ après choix d'une base de Lie $G$. Puisque pour tout $x \in \stackrel{\circ}{B}^{d}(0, \epsilon)$, $\lim _{n \rightarrow+\infty} p^{n} x=0$, on a

$$
U=\bigcup_{n \geq 1} p^{-n} V
$$

qui est donc un sous-groupe ouvert de $G^{a n}$. Posant $(\log )_{\mid p^{-n} V}=p^{-n} \log \circ\left(\times p^{n}\right)$ on définit ainsi par recollement un morphisme de groupes $\log : U \longrightarrow \mathbb{G}_{a}^{a n} \otimes$ Lie $G$. Le morphisme induit au niveau des espaces rigides est étale puisque l'application induite au niveau des algèbres de Lie est un isomorphisme. C'est en particulier un isomorphisme au voisinage de l'élément neutre.

Soit $\operatorname{ker}(\log ) \subset U$, un sous-groupe analytique Zariski fermé dans $U$. Puisque $\log ^{\text {rig }}$ est étale c'est un groupe étale sur $K$ et on a bien $\operatorname{ker}(\log )=G\left[p^{\infty}\right]$.

Exemple 5. (1) Soit $G=\mathbb{G}_{m}^{\text {rig }}$. Alors $U=\widehat{\mathbb{G}}_{m}^{a n}=\left\{x \in \mathbb{G}_{m}^{\text {rig }}|| x-1 \mid<1\right\}$.

(2) Plus généralement soit $T$ un tore algébrique sur $K$ et $X^{*}(T)$ son groupe des cocaractères. Alors $U=\left\{x \in T^{\text {an }}\left|\forall \chi \in X^{*}(T),\right| 1-\chi(x) \mid<1\right\}$.

(3) Soit $\mathcal{G}$ un groupe commutatif dans la catégorie des schémas formels formellement de type fini sur $\operatorname{Spf}\left(\mathcal{O}_{K}\right)$. Supposons le plat sur $\mathcal{O}_{K}$. Notons $\mathcal{G}_{s}$ sa fibre spéciale réduite et $\mathrm{sp}:\left|\mathcal{G}^{a n}\right| \longrightarrow$ $\left|\mathcal{G}_{s}\right|$ l'application de spécialisation. Alors

$$
U=\bigcup_{n \geq 1} \operatorname{sp}^{-1}\left(\mathcal{G}_{s}\left[p^{n}\right]\right) .
$$

(4) Par exemple si $\mathcal{G}$ est un groupe formel (formellement lisse) commutatif sur $\operatorname{Spf}\left(\mathcal{O}_{K}\right)$ alors $U=\mathcal{G}^{a n}$ et $\log _{U}$ est donné par le logarithme du groupe formel $\mathcal{G} \otimes K$.

(5) Si la valuation de $K$ est discrète, $A$ est une variété abélienne sur $K$ et $\mathcal{A}$ son modèle de Néron sur $\mathcal{O}_{K}$, soit $\widehat{\mathcal{A}}$ le complété p-adique de $\mathcal{A}$. Soit $U \subset A^{\text {an }}$. Le sous-groupe $\widehat{\mathcal{A}}^{\text {an }} \subset A^{\text {an }}$ est un sous-groupe analytique compact qui contient $U$ et $U=\bigcup_{n \geq 1} \operatorname{sp}^{-1}\left(\mathcal{A}_{s}\left[p^{n}\right]\right)$.

1.7. Sur les modèles de Néron des groupes analytiques rigides affinoïdes. 
1.7.1. Rappels de quelques résultats de Grauert et Remmert. Soit $A$ une $K$-algèbre affinoïde réduite. On note

$$
A^{0}=\left\{a \in A \mid\|a\|_{\infty} \leq 1\right\}
$$

l'anneau des éléments dont les puissances sont bornées. Il s'agit d'une $\mathcal{O}_{K}$-algèbre $p$-adiquement séparée complète telle que $A^{0}\left[\frac{1}{p}\right]=A$. De plus si $\mathcal{A} \subset A^{0}$ est une sous- $\mathcal{O}_{K}$-algèbre $p$-adiquement séparée complète topologiquement de type fini telle que $A=\mathcal{A}\left[\frac{1}{p}\right]$ alors $A^{0}$ est la fermeture intégrale de $\mathcal{A}$ dans $A$. La $\mathcal{O}_{K}$-algèbre $p$-adique $A^{0}$ fournit donc « un modèle entier canonique $\gg$ de $A$, c'est le normalisé de n'importe quel modèle de $A$ dans sa fibre générique. Sauf que ce modèle n'est pas en général topologiquement de type fini. Rappelons néanmoins le théorème suivant de Grauert et Remmert (cf. les corollaires 5 et 6 la section 6.4.1 de [6]) :

Théorème 1.3 (Grauert-Remmert). Supposons la valuation de $K$ discrète ou bien $K$ algébriquement clos. La $\mathcal{O}_{K}$-algèbre p-adique $A^{0}$ est alors topologiquement de type fini.

D'après la proposition 1.1 de [7] elle est de plus topologiquement de présentation finie c'est à dire isomorphe à une algèbre de la forme $\mathcal{O}_{K}<T_{1}, \ldots, T_{n}>/ I$ avec $I$ un idéal de type fini. Notons maintenant $k_{K}$ le corps résiduel de $K$. On note

$$
\widetilde{A}=A^{0} / A^{00}
$$

où $A^{00}=\left\{a \in A \mid\|a\|_{\infty}<1\right\}$ l'idéal de $A^{0}$ formé des éléments topologiquement nilpotents de $A$. La $k_{K}$-algèbre $\widetilde{A}$ est réduite de type fini (et ce quel que soit $K,[6] 6.4 .3 / 3$ ). Il y a une application de réduction surjective

$$
A^{0} \otimes k_{K} \rightarrow \widetilde{A}
$$

qui identifie $\widetilde{A}$ avec le réduit de $A^{0} \otimes k_{K}$. En particulier $A^{0} \otimes k_{K}=\widetilde{A}$ si et seulement si $A^{0} \otimes k_{K}$ est réduite. C'est toujours le cas si la valuation de $K$ n'est pas discrète. Lorsque cette valuation est discrète c'est le cas si et seulement si $\|A\|_{\infty}=|K|$.

Soit un morphisme surjectif

$$
\pi: K<X_{1}, \ldots, X_{n}>\rightarrow A \text {. }
$$

À un tel morphisme associons $\|.\|_{\pi}$ la norme résiduelle sur $A$ déduite de la norme de Gauss sur $K<X_{1}, \ldots, X_{n}>$ et $A_{\pi}$ sa boule unité, une $\mathcal{O}_{K}$-algèbre $p$-adiquement séparée complète ouverte dans $A$. On a d'après le corollaire $5.2 .7 / 8$ de $[6]$

$$
A_{\pi}=\pi\left(\mathcal{O}_{K}<X_{1}, \ldots, X_{n}>\right)
$$

qui est donc une $\mathcal{O}_{K}$-algèbre topologiquement de type fini. Réciproquement, les sous-algèbres de $A$ de la forme $A_{\pi}$ sont exactement les sous-algèbres $p$-adique $\mathcal{A}$ de $A^{0}$ topologiquement de type fini telles que $A=\mathcal{A}\left[\frac{1}{p}\right]$. Rappelons qu'un tel morphisme $\pi$ est dit distingué si la norme résiduelle d'algèbre de Banach $\|.\|_{\pi}$ induite sur $A$ coïncide avec la norme sup $\|.\|_{\infty} \operatorname{sur} A$.

Proposition 10 ([6], section 6.4.3). Les conditions suivantes sont équivalentes :

(1) $\pi$ est distingué

(2) la $k_{K}$-algèbre $A_{\pi} \otimes k_{K}$ est réduite

(3) $\|A\|_{\infty}=|K|$ et $\pi: \mathcal{O}_{K}<X_{1}, \ldots, X_{n}>\longrightarrow A^{0}$ est surjectif

De cela on déduit :

Corollaire 5. Soit $\mathcal{A}$ une sous- $\mathcal{O}_{K}$-algèbre p-adique de $A^{0}$ topologiquement de type fini telle que $A=\mathcal{A}\left[\frac{1}{p}\right]$. Si $\mathcal{A} \otimes k_{K}$ est réduite alors $\mathcal{A}=A^{0}$.

1.7.2. Les modèles normaux stables des algèbres affinoüdes.

Proposition 11. (1) Soit $A$ une $K$-algèbre affinö̈de réduite telle que $A^{0}$ soit topologiquement de type fini et $A \otimes k_{K}$ soit géométriquement réduite. Alors pour toute extension valuée complète $L$ de $K$

$$
(A \hat{\otimes} L)^{0}=A^{0} \underset{\mathcal{O}_{K}}{\hat{\otimes}} \mathcal{O}_{L}
$$

où dans le membre de droite $\hat{\otimes}$ signifie le produit tensoriel complété pour la topologie $p$ adique. 
(2) Soient $A$ et $\mathrm{B}$ deux $K$-algèbres affinoïdes réduites telles que $A^{0}$ et $\mathrm{B}^{0}$ soient topologiquement de type fini et $A^{0} \otimes k_{K}$ et $\mathrm{B}^{0} \otimes k_{K}$ soient géométriquement réduites. Alors

$$
(A \underset{K}{\hat{\otimes}} \mathrm{B})^{0}=A^{0} \underset{\mathcal{O}_{K}}{\hat{\otimes}} \mathrm{B}^{0}
$$

Démonstration. Il suffit d'appliquer le corollaire 5.

Théorème 1.4 ([8], th. 1.3). Il existe une extension de degré fini $L$ de $K$ telle que $\left(A_{L}\right)^{0}$ soit topologiquement de type fini et $\left(A_{L}\right)^{0} \otimes k_{L}$ géométriquement réduit.

Résumons tout cela dans le théorème suivant (pour la toute dernière assertion on utilise le corollaire $6.4 .1 / 5$ de $[6])$.

Théorème 1.5. Si $X$ est un espace rigide affinoïde réduit, il existe une extension de degré fini $L$ de $K$ telle que $X_{L}:=X \otimes_{K} L$ possède un modèle formel $\mathfrak{X}_{L}$ tel que

- $\mathfrak{X}_{L}$ est un schéma formel affine plat topologiquement de type fini sur $\operatorname{Spf}\left(\mathcal{O}_{L}\right)$ et $\mathfrak{X}_{L}^{\text {rig }}=X_{L}$

- La fibre spéciale de $\mathfrak{X}_{L}, \mathfrak{X}_{L} \otimes k_{L}$, est un schéma géométriquement réduit.

- Les deux précédentes conditions caractérisent $\mathfrak{X}_{L}$ à isomorphisme unique près.

- $\mathfrak{X}_{L}$ est intégralement clos dans sa fibre générique.

- Si $L^{\prime} \mid L$ est une extension valuée complète alors $X_{L^{\prime}}:=X \hat{\otimes}_{K} L^{\prime}$ possède également un tel modèle formel et

$$
\mathfrak{X}_{L^{\prime}}=\mathfrak{X}_{L} \hat{\mathbb{Q}}_{\mathcal{O}_{L}} \mathcal{O}_{L^{\prime}}
$$

- Si $Y$ est un autre espace rigide affinoïde réduit, $L \mid K$ est telle que $Y_{L}$ possède un modèle $\mathfrak{Y}_{L}$ du type précédent alors il en est de même de $(X \times Y)_{L}$ et ce modèle est

$$
\mathfrak{X}_{L} \times_{S p f\left(\mathcal{O}_{L}\right)} \mathfrak{Y}_{L}
$$

Cela définit une équivalence de catégories

germes d'espaces rigides affinoïdes réduits sur des extensions finies de $K$

$$
\left(X_{L}\right)_{L \mid K \text { grande }} \longmapsto\left(\mathfrak{X}_{L}\right)_{L \mid K \text { grande }}
$$

compatible aux produits.

En particulier si $K$ est algébriquement clos tout espace rigide affinoïde réduit possède un unique modèle formel affine plat topologiquement de type fini à fibre spéciale réduite. Le foncteur qui à un tel espace associe son modèle formel est compatible aux produits. Si $X \longrightarrow Y$ est un morphisme fini d'espaces affinoïdes réduits alors le morphisme entre leurs modèles $\mathfrak{X} \longrightarrow \mathcal{Y}$ est également fini.

1.7.3. Application aux groupes analytiques rigides affinoïdes.

Proposition 12. Soit $G$ un groupe analytique rigide affinö̈de sur $K$. Il existe alors une extension de degré fini $L$ de $K$ ainsi qu'un groupe $\mathcal{G}_{L}$ dans la catégorie des schémas formels topologiquement de type fini sur $\operatorname{Spf}\left(\mathcal{O}_{L}\right)$ tel que $\mathcal{G}_{L}$ soit affine et lisse sur $\operatorname{Spf}\left(\mathcal{O}_{L}\right)$ et $\mathcal{G}_{L}^{\text {rig }}=G_{L}$. De plus un tel groupe est unique à isomorphisme unique près.

Lorsque $K$ est algébriquement clos cela définit une équivalence entre la catégorie des groupes analytiques rigides affinö̈des et celle des schémas formels en groupes affines topologiquement de type fini et lisses sur $\operatorname{Spf}\left(\mathcal{O}_{K}\right)$.

Démonstration. Cela résulte de ce que tout schéma en groupes géométriquement réduit sur un corps est lisse.

Ainsi tout groupe analytique rigide affinoïde a potentiellement bonne réduction.

Proposition 13. Tout morphisme fini et surjectif de groupes analytiques rigides affinoïdes induit un morphisme fini et localement libre entre leurs modèles entiers canoniques. 
Démonstration. $\mathrm{Si} \mathrm{Sp}(\mathrm{B}) \longrightarrow \mathrm{Sp}(A)$ est un tel morphisme alors $A^{0} \longrightarrow \mathrm{B}^{0}$ est fini. De plus par surjectivité du morphisme de $\operatorname{spécialisation} \operatorname{Spec}(\widetilde{\mathrm{B}}) \longrightarrow \operatorname{Spec}(\widetilde{A})$ est un morphisme surjectif de schémas an groupes de type fini sur un corps et est donc plat. On conclut en utilisant que $A^{0}$ et $\mathrm{B}^{0}$ sont topologiquement de présentation finie sur $\mathcal{O}_{K}$.

Proposition 14. Soit $G$ un groupe analytique rigide affinö̈de commutatif connexe tel que $\forall g \in$ $G^{a n}, \lim _{n \rightarrow+\infty} p^{n} g=0$. Supposons $K$ algébriquement clos. Alors comme variété rigide, $G \simeq \mathbb{B}^{d}(0,1)$.

Démonstration. Soit $G=\operatorname{Sp}(A)$ et $\mathcal{G}=\operatorname{Spf}\left(A^{0}\right)$ son modèle lisse de fibre spéciale $\mathcal{G}_{s}=\operatorname{Spec}(\widetilde{A})$, un groupe commutatif lisse $\operatorname{sur} \operatorname{Spec}\left(k_{K}\right)$. Puisque $G$ est connexe, $\mathcal{G}_{s}$ l'est. Donc, $\mathcal{G}_{s}$ est isomorphe au produit d'un tore par un groupe unipotent lisse. Mais l'hypothèse $\forall g \in G^{a n}, \lim _{n \rightarrow+\infty} p^{n} g=0$ entraîne que pour $n \gg 0, \times p^{n}: \mathcal{G}_{s} \longrightarrow \mathcal{G}_{s}$ est le morphisme nul. Donc, $\mathcal{G}_{s}$ est un groupe unipotent lisse. Cela entraîne que comme variété algébrique $\mathcal{G}_{s} \simeq \mathbb{A}_{k_{K}}^{d}$. Le corollaire 6.4.2/3 de [6] permet de conclure.

\section{LES GROUPES ANALYTIQUES RIGIDES DE TYPE P-DIVISIBLE}

2.1. Définition. Soit $K \mid \mathbb{Q}_{p}$ un corps valué complet pour une valuation de rang 1 étendant la valuation $p$-adique. On prend la terminologie du chapitre 1 sur les espaces rigides et les espaces analytiques.

La définition qui suit est motivée de la façon suivante : on se propose de relâcher la condition d'être de $p$-torsion dans la définition d'un groupe $p$-divisible en la remplaçant pas la condition d'être $\ll$ topologiquement de $p$-torsion $\gg$.

Définition 2. Un $K$-groupe analytique rigide commutatif $G$ est dit de type p-divisible si :

(1) $\forall g \in G^{a n}, \lim _{n \rightarrow+\infty} p^{n} g=0$ pour la topologie de l'espace de Berkovich $\left|G^{a n}\right|$

(2) Le morphisme $G \stackrel{\times p}{\longrightarrow} G$ est fini et surjectif

Exemple 6. Tout groupe p-divisible $H$ sur $K$ définit un groupe analytique rigide de type p-divisible, $\lim H\left[p^{n}\right]^{\text {rig }}$. Si $V$ est un $K$-espace vectoriel de dimension fini $V \otimes \mathbb{G}_{a}^{\text {rig }}$ (confère exemple 1) est $\overrightarrow{n \geq 1}$

un groupe analytique rigide de type p-divisible. Si $\mathcal{G}$ est un groupe formel p-divisible sur $\operatorname{Spf}\left(\mathcal{O}_{K}\right)$ alors $\mathcal{G}^{\text {rig }}$ est de type $p$-divisible.

Proposition 15. Soit $G$ un $K$-groupe analytique rigide commutatif tel que $\forall g \in G^{a n}, \lim _{n \rightarrow+\infty} p^{n} g=$ 0. Il est de type p-divisible si et seulement si il vérifie les conditions suivantes :

(1) $G(\bar{K})[p]$ est fini

(2) Le morphisme $G^{a n} \stackrel{\times p}{\longrightarrow} G^{a n}$ est surjectif

(3) $G^{a n}$ est un bon espace analytique

(4) $\partial\left(G^{a n} / \mathcal{M}(K)\right)=\emptyset$

Démonstration. Supposons $G$ de type $p$-divisible. Le point $(1)$ est clair. Puisque $G \stackrel{\times p}{\longrightarrow}$ est un morphisme étale (cf. le lemme 1) et qu'il est par hypothèse fini, $G^{a n} \stackrel{\times p}{\longrightarrow} G^{a n}$ est étale fini. C'est donc un morphisme ouvert et fermé qui est donc surjectif si et seulement si il l'est au niveau des points « classiques $\gg$ de $G^{a n}$ (i.e. ceux de $G$ ). D'où le point (2). D'après la démonstration du théorème 1.2 il existe un voisinage ouvert $U$ de $e$ dans $G^{a n}$ qui forme un sous-groupe, qui est isomorphe à un sous-groupe ouvert de Lie $G \otimes \mathbb{G}_{a}^{a n}$ et tel que $G^{a n}=\bigcup_{n>1} p^{-n}(U)$. Puisque $U$ est isomorphe à un ouvert de Lie $G \otimes \mathbb{G}_{a}^{a n}$ qui est un bon espace analytique, $U$ est un bon espace analytique. Puisque pour tout $n \geq 1, \times p^{n}: p^{-n} U \longrightarrow U$ est étale fini on en déduit que $p^{-n} U$ est également un bon espace analytique. Donc $G^{a n}$ est un bon espace analytique, d'où le point (3). D'après [4] 1.5 .5 (ii), $p^{-1}\left(\operatorname{Int}\left(G^{a n} / K\right)\right) \cap \operatorname{Int}(\times p) \subset \operatorname{Int}\left(G^{a n} / K\right)$. Puisque $\times p: G^{a n} \longrightarrow G^{a n}$ est fini, $\operatorname{Int}(\times p)=G^{a n}$. Donc $p^{-1}\left(\operatorname{Int}\left(G^{a n} / K\right)\right) \subset \operatorname{Int}\left(G^{a n} / K\right)$. Mais si $U$ est un ouvert comme précédemment, puisque $U$ est un ouvert d'un espace affine, $\operatorname{Int}(U / K)=\emptyset$. Utilisant que $G=$ $\bigcup_{n \geq 1} p^{-n}(U)$ on conclut que $\operatorname{Int}\left(G^{a n} / K\right)=\emptyset$, d'où le point (4). 
Réciproquement, d'après [4] 1.5.5 (ii) puisque $G^{a n}$ est bon et séparé $p^{-1}\left(\operatorname{Int}\left(G^{a n} / K\right)\right) \cap \operatorname{Int}(\times p)=$ $\operatorname{Int}\left(G^{a n} / K\right)$. Le point (4) implique que $\operatorname{Int}\left(G^{a n} / K\right)=G^{a n}$. Donc $\partial(\times p)=\emptyset$. Le morphisme $\times p: G \longrightarrow G$ étant étale on en déduit que $\times p: G^{a n} \longrightarrow G^{a n}$ est étale surjectif. Il suffit alors d'appliquer le lemme 5 pour conclure que la multiplication par $p$ est un morphisme fini.

Définition 3. On note $\mathcal{R}_{K}$ la catégorie des $K$-groupes analytiques rigides de type $p$-divisible.

On vérifie immédiatement qu'il s'agit d'une catégorie additive. Si $\operatorname{pdiv}_{K}$ désigne la catégorie des groupes $p$-divisibles sur $K$ il y a un foncteur additif pleinement fidèle

$$
\begin{aligned}
\operatorname{pdiv}_{K} & \longrightarrow \mathcal{R}_{K} \\
H & \longmapsto \underset{n \geq 1}{\lim } H\left[p^{n}\right]^{r i g}
\end{aligned}
$$

Via ce foncteur on identifiera pdiv ${ }_{K}$ à une sous-catégorie pleine de $\mathcal{R}_{K}$. On notera qu'après choix d'une clôture algébrique $\bar{K}$ de $K$ il y a une équivalence de catégories

$$
\begin{aligned}
\operatorname{pdiv}_{K} & \stackrel{\sim}{\longrightarrow}\left\{\begin{array}{c}
\text { Représentations continues de } \operatorname{Gal}(\bar{K} \mid K) \text { dans des } \\
\mathbb{Z}_{p}-\text { modules libres de type fini }
\end{array}\right\} \\
H & \longmapsto T_{p}(H)
\end{aligned}
$$

Notons Vect ${ }_{K}$ la catégorie des $K$-espaces vectoriels de dimension finie. Il y a un foncteur pleinement fidèle

$$
\begin{aligned}
\operatorname{Vect}_{K} & \longrightarrow \mathcal{R}_{K} \\
V & \longmapsto V \otimes \mathbb{G}_{a}^{r i g}
\end{aligned}
$$

\subsection{Le morphisme logarithme.}

Proposition 16. Soit $G$ un groupe analytique rigide de type p-divisible. Il y a alors une suite exacte

$$
0 \longrightarrow G\left[p^{\infty}\right] \longrightarrow G \stackrel{\log }{\longrightarrow} \text { Lie } G \otimes \mathbb{G}_{a}^{r i g} \longrightarrow 0
$$

où $G\left[p^{\infty}\right]$ est un groupe $p$-divisible, $\log ^{a n}$ est un morphisme étale surjectif qui fait de $G^{\text {an }}$ un revêtement étale de Lie $G \otimes \mathbb{G}_{a}^{a n}$ au sens de De Jong.

Démonstration. Puisque $G[p]$ est fini sur $K$ et $\times p: G \longrightarrow G$ est surjectif, $G\left[p^{\infty}\right]$ est bien un groupe $p$-divisible. Le théorème 1.2 du chapitre 1 fournit un morphisme étale log : $G \longrightarrow$ Lie $G \otimes \mathbb{G}_{a}^{r i g}$. Puisque l'image de $\log ^{a n}$ contient un voisinage de 0 et $\times p: G^{a n} \longrightarrow G^{a n}$ est surjectif, $\log ^{a n}$ est surjectif. Il reste donc à voir que $\log ^{a n}$ est étale, sachant que log est étale. D'après [4] 1.5.5 (ii) puisque $G^{a n}$ est bon, $\operatorname{Int}\left(G^{a n} / K\right)=\left(\log ^{a n}\right)^{-1}\left(\operatorname{Int}\left(\operatorname{Lie} G \otimes \mathbb{G}_{a}^{a n}\right)\right) \cap \operatorname{Int}\left(\log ^{a n}\right)$. Puisque $\partial\left(\right.$ Lie $\left.G \otimes \mathbb{G}_{a}^{a n}\right)=\emptyset$, on en déduit que $\operatorname{Int}\left(\log ^{a n}\right)=\operatorname{Int}\left(G^{a n} / K\right)=G^{a n}$ et $\operatorname{donc} \partial\left(\log ^{a n}\right)=\emptyset$.

Il y a donc un foncteur

$$
\begin{aligned}
\mathcal{R}_{K} & \longrightarrow \operatorname{pdiv}_{K} \\
G & \longmapsto G\left[p^{\infty}\right]
\end{aligned}
$$

qui est adjoint à droite à l'inclusion naturelle pdiv $\operatorname{din}_{K} \longrightarrow \mathcal{R}$. Après choix d'une clôture algébrique de $\bar{K}$ de $K$ de complété $C$, via l'équivalence de catégories entre $\operatorname{pdiv}_{K}$ et les $\operatorname{Gal}(\bar{K} \mid K)$-modules continus libres de type fini sur $\mathbb{Z}_{p}$ ce foncteur s'identifie au foncteur

$$
\begin{aligned}
\mathcal{R}_{K} & \longrightarrow \operatorname{Rep}_{\mathbb{Z}_{p}}(\operatorname{Gal}(\bar{K} \mid K)) \\
G & \longmapsto \operatorname{Hom}\left(\mathbb{Q}_{p} / \mathbb{Z}_{p}, G \hat{\otimes} C\right)
\end{aligned}
$$

Il y a également un foncteur

$$
\begin{aligned}
\mathcal{R}_{K} & \longrightarrow \operatorname{Vect}_{K} \\
G & \longmapsto \operatorname{Lie} G
\end{aligned}
$$


qui est adjoint à gauche à l'inclusion $\operatorname{Vect}_{K} \longrightarrow \mathcal{R}$ définie par $V \mapsto V \otimes \mathbb{G}_{a}^{r i g}$. Ce foncteur s'identifie au foncteur

$$
G \longrightarrow \operatorname{Hom}\left(G, \mathbb{G}_{a}^{r i g}\right)^{*}
$$

où $\operatorname{Hom}\left(G, \mathbb{G}_{a}^{r i g}\right)$ est un $K$-espace vectoriel via l'action de $K$ sur $\mathbb{G}_{a}^{r i g}$ par homothéties.

Définition 4. Pour un groupe analytique rigide de type $p$-divisible $G$ on pose $\operatorname{dim} G=\operatorname{dim}$ Lie $G$ la dimension de l'espace rigide $G$ et

$$
\text { ht } G=\operatorname{ht} G\left[p^{\infty}\right]
$$

la hauteur du groupe p-divisible associé.

On dispose donc de deux fonctions

$$
\text { ht, } \operatorname{dim}: \mathcal{R}_{K} \longrightarrow \mathbb{N} \text {. }
$$

Corollaire 6. Les groupes analytiques rigides de type p-divisible de dimension 0 sont les groupes p-divisibles, ceux de hauteur 0 sont les groupes isomorphes aux groupes $\left(\mathbb{G}_{a}^{\text {rig }}\right)^{d}$ pour des $d \geq 0$.

2.3. Dévissage en limite inductive de groupes affinoïdes. Soit $G$ un $K$-groupe analytique rigide de type $p$-divisible tel que $d=\operatorname{dim} G>0$. Soit $U$ un sous-groupe affinoïde de $G$ tel que $\log _{\mid U}: U \stackrel{\sim}{\longrightarrow} \mathbb{B}^{d}(0, \epsilon)$ soit un isomorphisme pour un $\epsilon \in\left|K^{\times}\right|^{1 / \infty}$ après choix d'une base de Lie $G$ Notons pour tout $n \geq 0$

$$
U_{n}=p^{-n}(U) .
$$

Alors $G=\bigcup_{n \geq 0} U_{n}$ et il y a des diagrammes d'extensions

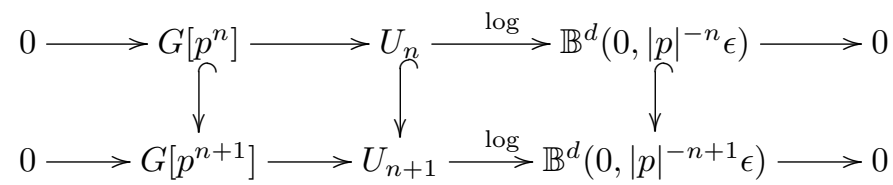

qui fournissent à la limite l'extension de la proposition 16 et où chaque membre de la limite inductive est un torseur sous un groupe fini au dessus d'une boule fermée d'un espace affine muni d'une structure de groupe compatible.

Plus généralement si $U$ est un sous-groupe affinoïde de $G$ il y a alors une suite exacte

$$
0 \longrightarrow G\left[p^{\infty}\right] \cap U \longrightarrow U \stackrel{\log _{\mid U}}{\longrightarrow} \log (U) \longrightarrow 0
$$

où $G\left[p^{\infty}\right] \cap U$ est fini, $\log (U)$ est un sous-groupe affinoïde de Lie $G \otimes \mathbb{G}_{a}^{\text {rig }}$ et $\log _{\mid U}: U \longrightarrow \log (U)$ est un morphisme étale fini. Notons $U_{n}=p^{-n} U$. Il y a alors des diagrammes de suites exactes pour $n \leq m$

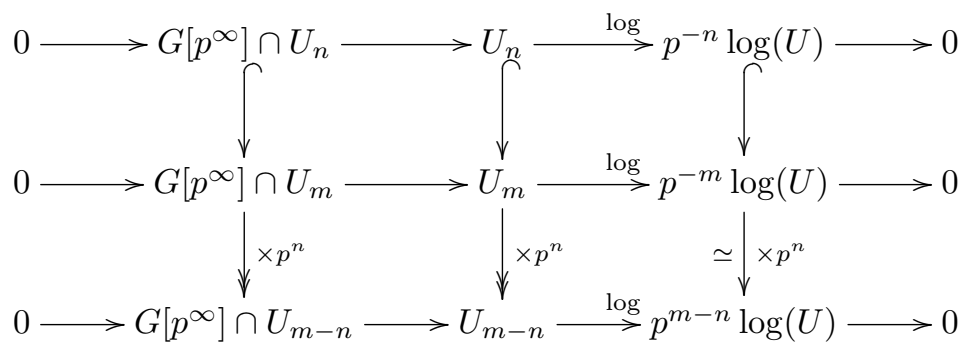


qui induisent des suites exactes

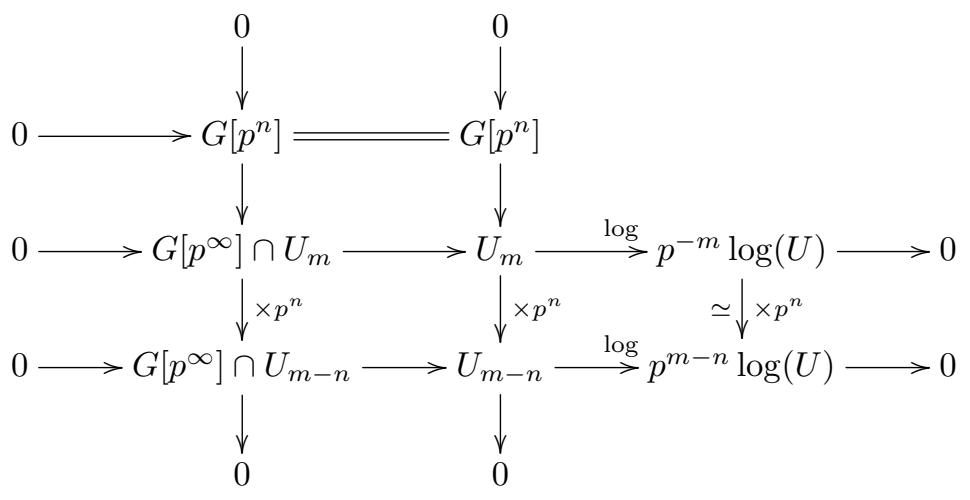

Remarquons également la proposition suivante.

Proposition 17. Supposons $K$ algébriquement clos et soit $G$ un $K$-groupe analytique rigide de type p-divisible. Soit $U \subset G$ un sous-groupe affinoïde. Alors comme espace rigide $U \simeq \mathbb{B}^{\operatorname{dim} G}(0,1)$.

Il s'agit d'une application de la proposition 14.

\subsection{Structure de $\mathbb{Z}_{p}$-module.}

Lemme 10. Tout groupe analytique rigide de type p-divisible est naturellement un $\mathbb{Z}_{p}$-module dans la catégorie des espaces rigides, de telle façon qu'après oubli de la structure $\mathbb{Z}_{p}$-linéaire on retrouve la structure de groupe abélien. Par «naturellement $\gg$ on entend que tout morphisme de groupe analytiques rigides est un morphisme de $\mathbb{Z}_{p}$-modules.

Démonstration. Commençons par constater que si $d \geq 1$ et $V \subset\left(\mathbb{G}_{a}^{r i g}\right)^{d}$ est un sous-groupe affinoïde alors $V$ est $\ll$ naturellement $\gg$ un $\mathbb{Z}_{p}$-module. Soit $\epsilon \in\left|K^{\times}\right|^{1 / \infty}$ tel que $V \subset \mathbb{B}^{d}(0, \epsilon)$. Soit $A$ une algèbre affinoïde réduite. Alors $V(A) \subset \mathbb{B}^{d}(0, \epsilon)(A)=\left\{a \in A \mid\|a\|_{\infty} \leq \epsilon\right\}$ est un fermé. Puisque $\mathbb{B}^{d}(0, \epsilon)(A)$ est $p$-adiquement séparé complet on en déduit que $V(A)$ est $p$-adiquement séparé complet. Le foncteur qui à une algèbre affinoïde réduite $A$ associe $V(A)$ est donc à valeurs dans les groupes abéliens $p$-adiquement séparés complets et donc puisque $V$ est représenté par une algèbre affinoïde réduite il est muni d'une structure de $\mathbb{Z}_{p}$-module.

On a maintenant $G=\underset{U}{\underset{U}{\longrightarrow}} U$ où $U$ parcourt les sous-groupes affinoïdes de $G$. Il suffit donc de définir une structure $\ll$ naturelle $\gg$ de $\mathbb{Z}_{p}$-module sur un tel $U$. Pour $n \gg 0, \log : p^{n} U \stackrel{\sim}{\longrightarrow} p^{n} \log (U)$.

Maintenant si $s \in U$ et $x \in \mathbb{Z}_{p}$, écrivons $x=a+p^{n} b$ avec $a \in \mathbb{Z}$ et $b \in \mathbb{Z}_{p}$. On pose alors $x . s=a . s+\log _{\mid p^{n} U}^{-1}\left(b . \log \left(p^{n} s\right)\right)$ dont on vérifie que cela ne dépend pas du choix de $a$ et $b$.

Exemple 7. Si $G=\widehat{\mathbb{G}}_{m}^{\text {rig }}=\stackrel{\circ}{\mathbb{B}}(1,1)$ et $A$ est une algèbre affinö̈de, $G(A)=1+A^{00}$ qui est un $\mathbb{Z}_{p}$-module via

$$
\forall x \in \mathbb{Z}_{p}, \forall a \in A^{00}, \quad(1+a)^{x}=\sum_{k \geq 0}\left(\begin{array}{l}
x \\
k
\end{array}\right) a^{k} .
$$

De plus le morphisme $\log : \widehat{G}_{m}^{\text {rig }} \longrightarrow \mathbb{G}_{a}^{\text {rig }}$ est un morphisme de $\mathbb{Z}_{p}$-module i.e.

$$
\log : 1+A^{00} \longrightarrow A
$$

est un morphisme de $\mathbb{Z}_{p}$-module.

Soit $G$ un groupe analytique rigide de type $p$-divisible et $\Lambda$ un $\mathbb{Z}_{p}$-module libre de type fini. On peut donc définir naturellement le foncteur $\Lambda \otimes_{\mathbb{Z}_{p}} G$ comme foncteur sur les $K$-algèbres affinoïdes. Il est représentable par une somme de copies de $G$ après choix d'une base de $\Lambda$. On notera que si $G=\mathbb{G}_{a}^{r i g}, \Lambda \otimes_{\mathbb{Z}_{p}} \mathbb{G}_{a}^{r i g}=\left(\Lambda \otimes_{\mathbb{Z}_{p}} K\right) \otimes \mathbb{G}_{a}^{r i g}$.

Corollaire 7. La catégorie $\mathcal{R}$ des groupes analytiques rigides de type p-divisible est une catégorie additive $\mathbb{Z}_{p}$-linéaire. 
2.5. Présentation comme extension d'un groupe additif par un groupe $p$-divisible. La proposition 16 admet la réciproque suivante.

Proposition 18. Soit $G$ un $K$-groupe analytique rigide commutatif tel qu'il existe une suite

$$
0 \longrightarrow \Gamma \longrightarrow G \stackrel{f}{\longrightarrow}\left(\mathbb{G}_{a}^{\text {rig }}\right)^{d} \longrightarrow 0
$$

où $\Gamma$ est un groupe p-divisible sur $K, f$ est un morphisme tel que ker $f$ s'identifie à $\Gamma$ et $f^{a n}$ est étale surjectif. Alors $G$ est de type $p$-divisible, $\Gamma=G\left[p^{\infty}\right]$ et si Lie $f:$ Lie $G \stackrel{\sim}{\longrightarrow} K^{d}$, le diagramme suivant commute

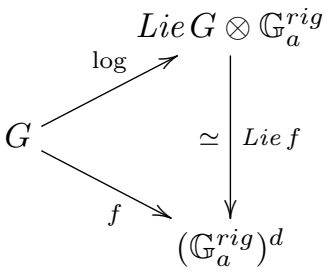

Démonstration. Soit $U$ un voisinage de 0 dans $G^{a n}$ qui forme un sous-groupe ouvert et tel que $\log _{\mid U}$ soit un isomorphisme sur son image. On a donc $\log ^{-1}(\log U) \simeq U \times \Gamma$. Soit $g \in G^{a n}$. Alors pour $n \gg 0, f\left(p^{n} g\right)=p^{n} f(g) \in \log (U)$. Donc, pour montrer que $p^{n} g \underset{n \rightarrow+\infty}{\rightarrow} 0$ il suffit de le montrer pour $g$ dans $\log ^{-1}(\log (U)) \simeq U \times \Gamma$ mais c'est alors clair.

La surjectivité de la multiplication par $p$ sur $G^{a n}$ ne pose pas de problème. On applique maintenant la proposition 15. Il est clair que $G(\bar{K})[p]$ est fini. Du fait que $f^{a n}$ est étale surjectif et $\left(\mathbb{G}_{a}^{a n}\right)^{d}$ un bon espace analytique on en déduit que $G$ est un bon espace analytique. Par application de [4] 1.5.5 (ii) au morphisme $f^{a n}$ on vérifie que $\partial\left(G^{a n} / K\right)=\emptyset$. Le reste ne pose pas de problème.

On peut même aller plus loin que la proposition précédent. Pour cela si $V$ est un $K$-espace vectoriel de dimension finie et $\Gamma$ un groupe $p$-divisible posons

$$
\operatorname{Ext}_{l o c . s c .}\left(V \otimes \mathbb{G}_{a}^{a n}, \Gamma\right)=\bigcup_{U} \operatorname{ker}\left(\operatorname{Ext}^{1}\left(V \otimes \mathbb{G}_{a}^{a n}, \Gamma\right) \longrightarrow \operatorname{Ext}^{1}(U, \Gamma)\right)
$$

où $U$ parcourt les sous-groupes ouverts de $V \otimes \mathbb{G}_{a}^{a n}$ et dans le membre de droite Ext ${ }^{1}$ signifie les extensions de faisceaux étales sur le gros site étale de $\mathcal{M}(K)$. Ce groupe classifie les extensions de $V \otimes \mathbb{G}_{a}^{a n}$ par $\Gamma$ qui sont scindées sur un sous-groupe ouvert de $V \otimes \mathbb{G}_{a}^{a n}$.

Proposition 19. Soit une extension de faisceaux étales de groupes abéliens

$$
0 \longrightarrow \Gamma \longrightarrow \mathcal{G} \stackrel{f}{\longrightarrow} V \otimes \mathbb{G}_{a}^{a n} \longrightarrow 0
$$

qui est scindée sur un sous-groupe ouvert de $V \otimes \mathbb{G}_{a}^{a n}$. Alors $\mathcal{G}$ est représenté par un groupe analytique associé à un groupe analytique rigide de type p-divisible.

Démonstration. Soit $U$ un sous-groupe ouvert de $V \otimes \mathbb{G}_{a}^{a n}$ et $s: U \longrightarrow \mathcal{G}$ une section de $f$ sur $U$. Pour tout $n \geq 0$ soit $U_{n}$ le sous-faisceau de $\mathcal{G}$ définit par le carré cartésien

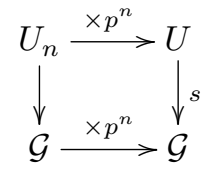

Il y a des suites exactes

$$
0 \longrightarrow \Gamma\left[p^{n}\right] \longrightarrow U_{n} \stackrel{f}{\longrightarrow} p^{-n} U \longrightarrow 0
$$

De la proposition 20 qui suit on déduit que $U_{n}$ est représenté par un groupe analytique et que $U_{n} \subset U_{n+1}$ est un ouvert. Il est alors facile de vérifier que $\underset{n}{\lim } U_{n}=\mathcal{G}$ et que le groupe analytique rigide associé au groupe analytique $\underset{n}{\lim } U_{n}$ vérifie les hypothèses de la proposition 18 . 
Proposition 20. Soit $X$ un $K$-espace analytique, $\Gamma$ un groupe étale fini sur $\mathcal{M}(K)$ et $\mathcal{F}$ un faisceau étale sur $X$ muni d'une action de $\Gamma$ qui en fait un $\Gamma$-torseur i.e. $\Gamma_{X} \times \mathcal{F} \stackrel{\sim}{\longrightarrow} \mathcal{F} \times \mathcal{F}$. Alors $\mathcal{F}$ est représentable par un $X$-espace analytique étale fini.

Démonstration. On vérifie que quitte à faire une extension de degré fini on peut supposer que $\Gamma$ est un groupe étale constant (il n'y a pas de problèmes à descendre les espaces analytiques étales finis sur $X \otimes_{K} L$ à $X$ lorsque $L \mid K$ est finie). Soit $x \in X$ et

$$
i_{x}: \mathcal{M}(\mathcal{K}(x)) \longrightarrow X
$$

le morphisme canonique associé. Le site $\mathcal{M}(\mathcal{K}(x))$ ét est formé des $\mathcal{K}(x)$-espaces analytiques isomorphes à un espace de la forme $\coprod_{i \in I} \mathcal{M}\left(L_{i}\right)$ avec $I$ quelconque et pour tout $i, L_{i} \mid \mathcal{K}(x)$ finie. Le faisceau $i_{x}^{*} \mathcal{F}$ est un $\Gamma$-torseur étale sur $\mathcal{M}(\mathcal{K}(x))$ et est donc représentable par un $\mathcal{K}(x)$-espace analytique étale fini galoisien de groupe $\Gamma$. D'après le théorème 3.4.1 de [4] cet espaces analytique correspond à un germe d'espaces analytiques $f: U \longrightarrow V$ où $V$ est un voisinage ouvert de $x, f$ est étale fini galoisien de groupe $\Gamma$. On a

$$
\operatorname{Hom}\left(i_{x}^{*} U, i_{x}^{*} \mathcal{F}\right)=\underset{V^{\prime}}{\lim _{\longrightarrow}} \mathcal{F}\left(f^{-1}\left(V^{\prime}\right)\right)
$$

où $V^{\prime}$ parcourt les voisinages ouverts de $x$ dans $V$ et puisque $\Gamma$ est fini

$$
\operatorname{Hom}_{\Gamma}\left(i_{x}^{*} U, i_{x}^{*} \mathcal{F}\right)=\lim _{V^{\prime}} \mathcal{F}\left(f^{-1}\left(V^{\prime}\right)\right)^{\Gamma}
$$

où $\Gamma$ agit sur $\mathcal{F}\left(f^{-1}\left(V^{\prime}\right)\right)^{\Gamma}$ via son action sur $\mathcal{F}$ et celle sur $f^{-1}\left(V^{\prime}\right)$. L'isomorphisme canonique $\Gamma$ équivariant $i_{x}^{*} U \stackrel{\sim}{\longrightarrow} i_{x}^{*} \mathcal{F}$ provient donc d'un morphisme $\Gamma$ équivariant $f^{-1}\left(V^{\prime}\right) \longrightarrow \mathcal{F}_{\mid V^{\prime}}$ pour $V^{\prime}$ suffisamment petit. Étant donné que tout morphisme de torseurs est un isomorphisme on en déduit que $f^{-1}\left(V^{\prime}\right) \stackrel{\sim}{\longrightarrow} \mathcal{F}_{\mid V^{\prime}}$.

Donc, localement sur $|X|, \mathcal{F}$ est représentable par un espace analytique étale fini. Il l'est donc globalement.

Théorème 2.1. Soit $\mathcal{C}$ la catégorie dont les objets sont les triplets $(V, \Gamma, \xi)$ où $V$ est un $K$-espace vectoriel de dimension finie, $\Gamma$ un groupe p-divisible et $\xi \in \operatorname{Ext}_{\text {loc.sc. }}\left(V \otimes \mathbb{G}_{a}^{a n}, \Gamma\right)$ et telle que $\operatorname{Hom}_{\mathcal{C}}\left((V, \Gamma, \xi),\left(V^{\prime}, \Gamma^{\prime}, \xi^{\prime}\right)\right)$ soit le sous-groupe de $\operatorname{Hom}\left(V, V^{\prime}\right) \oplus \operatorname{Hom}\left(\Gamma, \Gamma^{\prime}\right)$ formé des $(f, h)$ tels que $h_{*} \xi=f^{*} \xi^{\prime}$. Le foncteur de la catégorie des groupes analytiques rigides de type $p$-divisibles vers $\mathcal{C}$ qui à $G$ associe $\left(G\left[p^{\infty}\right]\right.$, Lie $\left.G, \xi_{\log }\right)$ où $\xi_{\log }$ est la classe donnée par l'extension de la proposition 16 induit une équivalence de catégories.

Démonstration. Après application de la proposition 20 il suffit de constater que la catégorie de telles extensions est rigide puisque si $\Gamma$ est un groupe $p$-divisible et $V$ un $K$-espace vectoriel de dimension finie alors $\operatorname{Hom}\left(V \otimes \mathbb{G}_{a}^{r i g}, \Gamma\right)=0$.

2.6. Isogénies. On vérifie facilement le lemme qui suit.

Lemme 11. Si $f: G \longrightarrow G^{\prime}$ est un morphisme de groupes analytiques rigides de type p-divisible sont équivalents :

- $f$ est une isogénie

- Il existe $n \in \mathbb{N}$ et $g: G^{\prime} \longrightarrow G$ tels que $f \circ g=p^{n}$ et $g \circ f=p^{n}$

- Lie $(f):$ Lie $G \stackrel{\sim}{\longrightarrow}$ Lie $G^{\prime}$ est un isomorphisme et $f: G\left[p^{\infty}\right] \longrightarrow G^{\prime}\left[p^{\infty}\right]$ est une isogénie

On peut alors définir la catégorie des groupes analytiques rigides de type $p$-divisible à isogénie. Il s'agit de la catégorie additive obtenue en inversant les flèches multiplication par $p$. Ses objets sont les groupes analytiques rigides de type $p$-divisibles et les morphismes entre $G$ et $G^{\prime}$ sont $\operatorname{Hom}\left(G, G^{\prime}\right) \otimes_{\mathbb{Z}_{p}} \mathbb{Q}_{p}$.

Définition 5. On note $\mathcal{R}_{K} \otimes \mathbb{Q}$ la catégorie des $K$-groupes analytiques rigides de type p-divisible à isogénie près. 
$\operatorname{Si~pdiv}_{K} \otimes \mathbb{Q}$ désigne la catégorie des groupes $p$-divisibles sur $K$ à isogénie près il y a donc un foncteur pleinement fidèle

$$
\operatorname{pdiv}_{K} \otimes \mathbb{Q} \longrightarrow \mathcal{R}_{K} \otimes \mathbb{Q}
$$

qui admet le foncteur $G \mapsto G\left[p^{\infty}\right]$ comme adjoint à droite. Après choix d'une clôture algébrique de $\bar{K}$ de $K$, si $\operatorname{Rep}_{\mathbb{Q}_{p}}\left(G_{K}\right)$ désigne la catégorie des représentations continues de $\operatorname{Gal}(\bar{K} \mid K)$ dans des $\mathbb{Q}_{p}$-espaces vectoriels de dimension finie, cela définit un foncteur pleinement fidèle

$$
\operatorname{Rep}_{\mathbb{Q}_{p}}\left(G_{K}\right) \longrightarrow \mathcal{R} \otimes \mathbb{Q}
$$

d'adjoint à droite $G \mapsto V_{p}\left(G\left[p^{\infty}\right]\right)$. De même le foncteur $V \mapsto V \otimes \mathbb{G}_{a}^{r i g}$ se factorise en un foncteur pleinement fidèle

$$
\text { Vect }_{K} \longrightarrow \mathcal{R} \otimes \mathbb{Q}
$$

qui admet le foncteur $G \mapsto \operatorname{Lie} G$ comme adjoint à gauche.

2.7. Opérations sur les groupes analytiques rigides de type $p$-divisible. Soit $G$ un $K$ groupe analytique rigide de type $p$-divisible.

2.7.1. Poussé en avant. Soit $\Gamma$ un groupe $p$-divisible sur $K$ et $f: G\left[p^{\infty}\right] \longrightarrow \Gamma$ un morphisme. Soit le faisceau étale quotient

$$
f_{*} G^{a n}:=G^{a n} \underset{G\left[p^{\infty}\right]}{\oplus} \Gamma .
$$

Il y a donc un diagramme de suites exactes

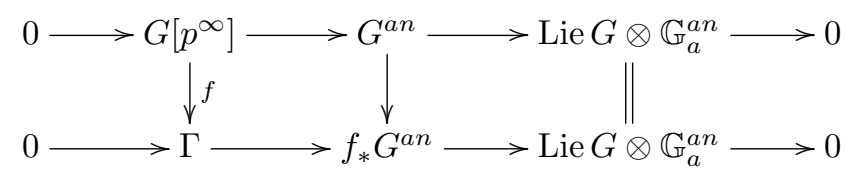

et la suite du bas est obtenue par image directe de celle du haut via $f$. Alors $f_{*} G^{a n}$ est représenté par un groupe analytique de type $p$-divisible. Il y a deux façons de le voir :

— Le théorème 1.1 et la remarque 5 montrent que $f_{*} G^{a n}=\left(G^{a n} \oplus \Gamma\right) / G\left[p^{\infty}\right]$ est représenté par un groupe analytique. La proposition 19 montre que ce groupe est de type $p$-divisible.

- Il suffit de vérifier que le morphisme $f_{*}: \operatorname{Ext}\left(\operatorname{Lie} G \otimes \mathbb{G}_{a}^{a n}, G\left[p^{\infty}\right]\right) \longrightarrow \operatorname{Ext}\left(\operatorname{Lie} G \otimes \mathbb{G}_{a}^{a n}, \Gamma\right)$ envoie $\operatorname{Ext}_{\text {loc.sc. }}\left(\operatorname{Lie} G \otimes \mathbb{G}_{a}^{a n}, G\left[p^{\infty}\right]\right)$ dans $\operatorname{Ext}_{\text {loc.sc. }}\left(\operatorname{Lie} G \otimes \mathbb{G}_{a}^{a n}, \Gamma\right)$.

On peut donc définir $f_{*} G$ comme groupe analytique rigide de type $p$-divisible. Par exemple si $\Gamma=(0)$ alors $f_{*} G=$ Lie $G \otimes \mathbb{G}_{a}^{r i g}$. On remarquera que si $f$ est une isogénie alors $G \longrightarrow f_{*} G$ en est également une. Si $f$ se factorise en $G\left[p^{\infty}\right] \stackrel{g}{\rightarrow} \Gamma^{\prime} \hookrightarrow \Gamma^{\prime} \oplus \Gamma^{\prime \prime}=\Gamma$ alors $f_{*} G=G / \operatorname{ker} g \oplus \Gamma^{\prime \prime}$. Quitte à faire une isogénie on peut toujours se ramener à ce cas là.

2.7.2. Tiré en arrière. Soit $f: V \longrightarrow \operatorname{Lie} G$ un morphisme de $K$-espaces vectoriels de dimension finie qui induit $f: V \otimes \mathbb{G}_{a}^{r i g} \longrightarrow \operatorname{Lie} G \otimes \mathbb{G}_{a}^{r i g}$. On peut alors définir

$$
f^{*} G=G \underset{\text { Lie } G \otimes \mathbb{G}_{a}^{r i g}}{\times} V \otimes \mathbb{G}_{a}^{r i g}
$$

qui est un groupe analytique rigide de type $p$-divisible s'inscrivant dans un diagramme

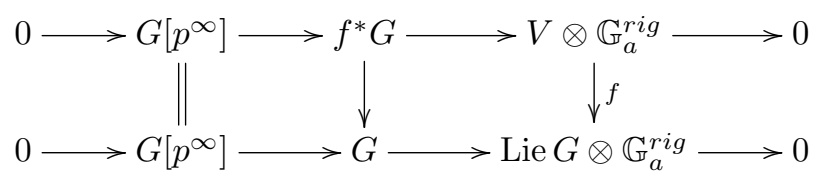

Si $V^{\prime} \subset V$ est un supplémentaire de ker $f$ alors

$$
f^{*} G \simeq V^{\prime} \otimes \mathbb{G}_{a}^{r i g} \oplus \log ^{-1}(\operatorname{Im} f)
$$

où $\log ^{-1}(\operatorname{Im} f)$ est un sous-groupe Zariski fermé de $G$ obtenu par image réciproque d'un sous-espace linéaire de Lie $G$ par le logarithme. 
2.7.3. Sommes.

Lemme 12. (1) Si $\Gamma$ est un groupe $p$-divisible, $V$ et $V^{\prime}$ sont deux $K$-espaces vectoriels de dimension finie

$$
\operatorname{Ext}_{\text {loc.sc. }}\left(V \otimes \mathbb{G}_{a}^{a n}, \Gamma\right) \oplus \operatorname{Ext}_{\text {loc.sc. }}\left(V^{\prime} \otimes \mathbb{G}_{a}^{a n}, \Gamma\right) \stackrel{\sim}{\longrightarrow} \operatorname{Ext}_{\text {loc.sc. }}\left(\left(V \oplus V^{\prime}\right) \otimes \mathbb{G}_{a}^{a n}, \Gamma\right)
$$

(2) Si $\Gamma$ et $\Gamma^{\prime}$ sont deux groupes $p$-divisibles et $V$ est un $K$-espace vectoriel de dimension finie

$$
\operatorname{Ext}_{\text {loc.sc }}\left(V \otimes \mathbb{G}_{a}^{a n}, \Gamma \oplus \Gamma^{\prime}\right) \stackrel{\sim}{\longrightarrow} \operatorname{Ext}_{\text {loc.sc. }}\left(V \otimes \mathbb{G}_{a}^{a n}, \Gamma\right) \oplus \operatorname{Ext}_{\text {loc.sc. }}\left(V \otimes \mathbb{G}_{a}^{a n}, \Gamma^{\prime}\right)
$$

Corollaire 8. Supposons $K$-algébriquement clos. Si $\Gamma$ est un groupe p-divisible et $V$ est un $K$ espace vectoriel de dimension finie il y a des isomorphismes canoniques

$$
\begin{aligned}
\operatorname{Ext}_{\text {loc.sc. }}\left(V \otimes \mathbb{G}_{a}^{a n}, \Gamma\right) & \simeq \operatorname{Ext}_{\text {loc.sc. }}\left(\mathbb{G}_{a}^{a n}, \mathbb{Q}_{p} / \mathbb{Z}_{p}\right) \otimes_{K} \operatorname{Hom}\left(V, T_{p}(G) \otimes_{\mathbb{Z}_{p}} K\right) \\
& \simeq \operatorname{Ext} t_{\text {loc.sc. }}\left(\mathbb{G}_{a}^{a n}, \mu_{p^{\infty}}\right) \otimes_{K} \operatorname{Hom}\left(V, T_{p}(G)(-1) \otimes_{\mathbb{Z}_{p}} K\right)
\end{aligned}
$$

2.8. Composantes connexes. Soit $G$ un groupe analytique rigide de type $p$-divisible et $\underline{\pi}_{0}(G)$ le groupe étale de ses composantes connexes (cf. appendice 1.4).

Proposition 21. Le groupe étale $\underline{\pi}_{0}(G)$ est un groupe p-divisible et le morphisme composé $G\left[p^{\infty}\right] \hookrightarrow$ $G \longrightarrow \underline{\pi}_{0}(G)$ est un épimorphisme. La composante connexe neutre $G^{0}$ est un groupe analytique rigide de type p-divisible.

Démonstration. Puisque la multiplication par $p, G \stackrel{\times p}{\longrightarrow} G$, est un morphisme étale fini surjectif elle induit un morphisme étale fini surjectif $\times p: \underline{\pi}_{0}(G) \longrightarrow \underline{\pi}_{0}(G)$. Si $X$ est une composante connexe de $G \hat{\otimes} \hat{\bar{K}}$ et $x \in X$, puisque $\lim _{n \rightarrow+\infty} p^{n} x=0$ dans $G^{a n} \hat{\otimes} \overline{\hat{K}}$, pour $n \gg 0$ on a $p^{n} x \in G^{0} \hat{\otimes} \widehat{\bar{K}}$. Donc pour $n \gg 0, p^{n} X \cap\left(G^{0} \hat{\otimes} \widehat{\bar{K}}\right) \neq \emptyset$ et donc par connexité de $p^{n} X, p^{n} X \subset G^{0} \hat{\otimes} \widehat{\bar{K}}$. Cela implique $\underline{\pi}_{0}(G)$ est de $p$-torsion. On en déduit que $\underline{\pi}_{0}(X)$ est un groupe $p$-divisible.

De plus si $X$ est comme précédemment et $n$ tel que $p^{n} X \subset G^{0} \hat{\otimes} \widehat{\bar{K}}$, puisque la multiplication par $p^{n}$ est un morphisme étale fini et $G^{0} \hat{\otimes} \widehat{\bar{K}}$ connexe, $p^{n} X=G^{0} \hat{\otimes} \widehat{\bar{K}}$. On en déduit que le morphisme $G\left[p^{\infty}\right] \longrightarrow \underline{\pi}_{0}(G)$ est surjectif. Le reste ne pose pas de problème.

Corollaire 9. Si K est algébriquement clos tout groupe analytique rigide de type p-divisible est isomorphe à un groupe de la forme $\Gamma \oplus G$ où $\Gamma$ est un groupe p-divisible et $G$ est un groupe analytique rigide de type p-divisible connexe.

Lemme 13. Soit $\left(U_{n}\right)_{n \geq 0}$ une suite croissante de sous-groupes affinoïdes ouverts de $G$ telle que $G=\bigcup_{n \geq 0} U_{n}$. Alors $\left(U_{n}^{0}\right)_{n \geq 0}$ forme une suite croissante de sous-groupes affinoïdes ouverts de $G$ telle que $G^{0}=\bigcup_{n \geq 0} U_{n}^{0}$.

Démonstration. Soit $n \geq 0$. Puisque $\log _{\mid U_{n}}: U_{n} \longrightarrow$ Lie $G \otimes \mathbb{G}_{a}^{r i g}$ est étale fini

$$
\log \left(U_{n}\right)=\log \left(U_{n}^{0}\right) .
$$

Soit $V=\bigcup_{n \geq 0} U_{n}^{0}$, un sous-groupe ouvert connexe de $G$. On a donc $\log (V)=\operatorname{Lie} G \otimes \mathbb{G}_{a}^{\text {rig }}$ ce qui implique que $G=G\left[p^{\infty}\right] . V$. Si $\Gamma=G\left[p^{\infty}\right] \cap V$ on a donc $G=\coprod_{\bar{\gamma} \in G\left[p^{\infty}\right] / \Gamma} \gamma \cdot V$ d'où le résultat.

Lemme 14. Supposons $G$ connexe. Il existe alors un sous-groupe affinoïde ouvert $U$ de $G$ tel que pour tout $n \geq 0, p^{-n}(U)$ soit connexe.

Démonstration. D'après le lemme 13 il existe un sous-groupe affinoïde $U$ de $G$ tel que $U$ soit connexe et $G[p] \subset U$. Vérifions qu'un tel $U$ convient. Par récurrence il suffit de montrer que pour un tel $U, p^{-1}(U)$ est connexe. Mais le revêtement $\times p: p^{-1}(U) \longrightarrow U$ est étale fini et est un torseur sous $G[p]$. On a donc $p^{-1}(U)=G[p] \cdot\left(p^{-1}(U)\right)^{0}$. Mais $U$ étant connexe on a $G[p] \subset U \subset$ $\left(p^{-1}(U)\right)^{0}$. 
2.9. Facteurs directs additifs. Soit $G$ un groupe analytique rigide de type $p$-divisible.

Définition 6. Le groupe $G$ est dit sans facteur direct additif si $\operatorname{Hom}\left(\mathbb{G}_{a}^{r i g}, G\right)=0$.

Lemme 15. L'application

$$
\begin{aligned}
\operatorname{Hom}\left(\mathbb{G}_{a}^{r i g}, G\right) & \longrightarrow \operatorname{Lie} G \\
f & \longmapsto \operatorname{Lie}(f)(1)
\end{aligned}
$$

est injective.

Démonstration. Si $f: \mathbb{G}_{a}^{r i g} \longrightarrow G$ vérifie $\operatorname{Lie}(f)=0$ alors $\log \circ f: \mathbb{G}_{a}^{r i g} \longrightarrow \operatorname{Lie} G \otimes \mathbb{G}_{a}^{r i g}$ est nul et donc $f: \mathbb{G}_{a}^{r i g} \longrightarrow G\left[p^{\infty}\right]$ qui est donc nul puisque $\mathbb{G}_{a}^{r i g}$ est connexe.

Corollaire 10. Soit $W=\operatorname{Hom}\left(\mathbb{G}_{a}^{\text {rig }}, G\right) \subset$ Lie $G$. Il y a alors une section du logarithme au dessus de $W \otimes \mathbb{G}_{a}^{r i g}$

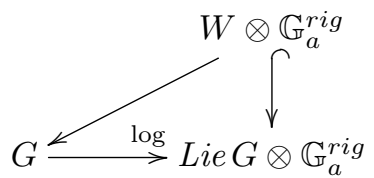

et $W \otimes \mathbb{G}_{a}^{r i g}$ est le plus grand sous-groupe additif de Lie $G \otimes \mathbb{G}_{a}^{\text {rig }}$ au dessus duquel il existe une telle section. De plus si $W^{\prime} \subset$ Lie $G$ est un supplémentaire de $W$ il y a un isomorphisme

$$
G \simeq\left(W \otimes \mathbb{G}_{a}^{r i g}\right) \oplus \log ^{-1}\left(W^{\prime} \otimes \mathbb{G}_{a}^{r i g}\right)
$$

qui fournit une décomposition de $G$ en une somme d'un groupe additif et d'un groupe analytique rigide de type p-divisible sans facteur direct additif.

2.10. Monomorphismes et épimorphismes de groupes analytiques rigides de type $p$ divisibles.

Proposition 22. Tout morphisme $f: G_{1} \longrightarrow G_{2}$ de $K$-groupes analytiques rigides de type pdivisibles induit des suites exactes de faisceaux sur le gros site étale de $S p(K)$

$$
0 \longrightarrow \operatorname{ker}\left(f_{\mid G_{1}\left[p^{\infty}\right]}\right) \longrightarrow \operatorname{ker} f \longrightarrow(\text { ker Lie } f) \otimes \mathbb{G}_{a}^{r i g} \longrightarrow 0
$$

et

$$
0 \longrightarrow \operatorname{coker}\left(G_{1}\left[p^{\infty}\right] \longrightarrow G_{2}\left[p^{\infty}\right]\right) \longrightarrow \operatorname{coker} f \longrightarrow(\text { coker Lie } f) \otimes \mathbb{G}_{a}^{\text {rig }} \longrightarrow 0
$$

Démonstration. On applique le lemme du serpent au diagramme de faisceaux étales de groupes abéliens

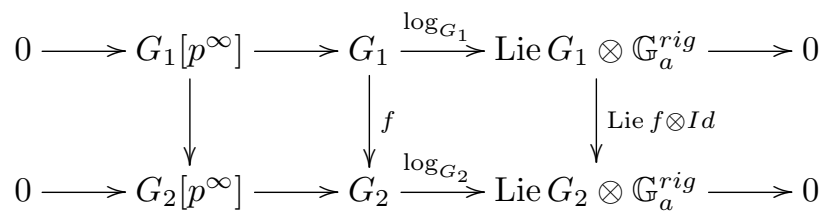

Le morphisme induit

$$
\operatorname{ker}(\operatorname{Lie} f \otimes I d)=\operatorname{ker}(\operatorname{Lie} f) \otimes \mathbb{G}_{a}^{r i g} \longrightarrow \operatorname{coker}\left(G_{1}\left[p^{\infty}\right] \longrightarrow G_{2}\left[p^{\infty}\right]\right)
$$

est nul puisque le membre de droite est représenté par un groupe analytique rigide étale $\operatorname{sur} \operatorname{Sp}(K)$ et celui de gauche est connexe. Le reste du lemme se déduit de ce que si $g: W \longrightarrow W^{\prime}$ est un morphisme de $K$-espaces vectoriels de dimension finie on vérifie facilement que comme faisceau sur le gros site étale de $\operatorname{Sp}(K)$ le conoyau du morphisme induit $W \otimes \mathbb{G}_{a}^{\text {rig }} \longrightarrow W^{\prime} \otimes \mathbb{G}_{a}^{\text {rig }}$ coïncide avec $(\operatorname{coker} g) \otimes \mathbb{G}_{a}^{r i g}$.

Corollaire 11. Soit $f: G_{1} \longrightarrow G_{2}$ un morphisme de groupes analytiques rigides de type pdivisibles. Sont alors équivalents :

- $f$ induit un épimorphisme de faisceaux sur le gros site étale de $S p(K)$

- $f$ est un morphisme lisse et $f_{\mid G_{1}\left[p^{\infty}\right]}: G_{1}\left[p^{\infty}\right] \longrightarrow G_{2}\left[p^{\infty}\right]$ est un épimorphisme de groupes p-divisibles i.e. $f_{*}: V_{p}\left(G_{1}\left[p^{\infty}\right]\right) \stackrel{\longrightarrow}{\longrightarrow} V_{p}\left(G_{2}\left[p^{\infty}\right]\right)$ est surjectif 
Corollaire 12. Soit $f: G_{1} \longrightarrow G_{2}$ un morphisme de groupes analytiques rigides de type pdivisibles. Sont alors équivalents :

- $f$ est un monomorphisme de faisceaux sur le gros site étale de $S p(K)$

- $f$ est un morphisme non ramifié et $f_{\mid G_{1}\left[p^{\infty}\right]}: G_{1}\left[p^{\infty}\right] \longrightarrow G_{2}\left[p^{\infty}\right]$ est un monomorphisme de groupes p-divisibles i.e. $f_{*}: T_{p}\left(G_{1}\left[p^{\infty}\right]\right) \longrightarrow T_{p}\left(G_{2}\left[p^{\infty}\right]\right)$ est un morphisme injectif faisant de $T_{p}\left(G_{1}\left[p^{\infty}\right]\right)$ un facteur direct de $T_{p}\left(G_{2}\left[p^{\infty}\right]\right)$

Corollaire 13. Une suite de groupes analytiques rigides de type p-divisibles

$$
0 \longrightarrow G^{\prime} \longrightarrow G \longrightarrow G^{\prime \prime} \longrightarrow 0
$$

est exacte comme suite de faisceaux étales sur $\operatorname{Sp}(K)$ si et seulement si

- La suite de $K$-espaces vectoriels $0 \longrightarrow$ Lie $G^{\prime} \longrightarrow$ Lie $G \longrightarrow$ Lie $G^{\prime \prime} \longrightarrow 0$ est exacte

- La suite de groupes $p$-divisibles $0 \longrightarrow G^{\prime}\left(\left[p^{\infty}\right]\right) \longrightarrow G\left[p^{\infty}\right] \longrightarrow G^{\prime \prime}\left[p^{\infty}\right] \longrightarrow 0$ est exacte

3. Classification des groupes analytiques Rigides De type $p$-Divisible Sur Un Corps ALGÉBRIQUEMENT CLOS

3.0.1. Un calcul d'extensions. Le calcul d'extensions de cette section est inspiré de celui de la section 9.4 de [9].

Soit $n \geq 1$ un entier. Soit $\mathbb{B}(0,1) \subset \mathbb{G}_{a}^{a n}$ comme sous-groupe analytique. Considérons le groupe d'extensions $\operatorname{Ext}^{1}\left(\mathbb{B}(0,1), \mu_{p^{n}}\right)$ sur le gros site étale de $\mathcal{M}(K)$. D'après la proposition 20 il paramètre les classes d'isomorphisme d'extensions de groupes analytiques

$$
0 \longrightarrow \mu_{p^{n}} \longrightarrow G \longrightarrow \mathbb{B}(0,1) \longrightarrow 0 .
$$

La suite exacte de Kümmer fournit une suite exacte

$$
\operatorname{Hom}\left(\mathbb{B}(0,1), \mathbb{G}_{m}^{a n}\right) \longrightarrow \operatorname{Ext}^{1}\left(\mathbb{B}(0,1), \mu_{p^{n}}\right) \longrightarrow \operatorname{Ext}^{1}\left(\mathbb{B}(0,1), \mathbb{G}_{m}^{a n}\right) \stackrel{\times p^{n}}{\longrightarrow} \operatorname{Ext}^{1}\left(\mathbb{B}(0,1), \mathbb{G}_{m}^{a n}\right)
$$

Intéressons nous maintenant au groupe $\operatorname{Ext}^{1}\left(\mathbb{B}(0,1), \mathbb{G}_{m}^{a n}\right)$. Puisque $\operatorname{Pic}(\mathbb{B}(0,1))$ est trivial les extensions associées possèdent une section comme morphisme d'espaces analytiques

$$
0 \longrightarrow \mathbb{G}_{m}^{a n} \longrightarrow \mathbb{\exists} \longrightarrow \mathbb{B}(0,1) \longrightarrow 0 .
$$

De là on déduit que $\operatorname{Ext}^{1}\left(\mathbb{G}_{m}^{a n}, \mathbb{B}(0,1)\right)$ est isomorphe à $H^{2}\left(C^{\bullet}\right)$ où $C^{\bullet}$ est le complexe

$$
C^{1} \stackrel{\partial}{\longrightarrow} C^{2} \stackrel{\partial}{\longrightarrow} C^{3}
$$

tel que

$$
\begin{aligned}
& C^{1}=\operatorname{Hom}\left(\mathbb{B}(0,1), \mathbb{G}_{m}^{a n}\right) \\
& C^{2}=\operatorname{Hom}\left((\mathbb{B}(0,1) \times \mathbb{B}(0,1)) / \mathfrak{S}_{2}, \mathbb{G}_{m}^{a n}\right) \\
& C^{3}=\operatorname{Hom}\left(\mathbb{B}(0,1) \times \mathbb{B}(0,1) \times \mathbb{B}(0,1), \mathbb{G}_{m}^{a n}\right)
\end{aligned}
$$

où Hom signifie les morphismes d'espaces analytiques, la loi de groupe est celle donnée par la loi de $\mathbb{G}_{m}^{a n}$ et l'action de $\mathfrak{S}_{2}$ sur $\mathbb{B}(0,1) \times \mathbb{B}(0,1)$ est la permutation des coordonnées. Les applications bord sont données par

$$
\begin{aligned}
\forall f \in C^{1}, \quad(\partial f)(x, y) & =f(x+y) f(x)^{-1} f(y)^{-1} \\
\forall g \in C^{2}, \quad(\partial g)(u, v, w) & =g(v, w) g(u+v, w)^{-1} g(u, v+w) g(u, v)^{-1} .
\end{aligned}
$$

Il s'identifie donc au complexe

$$
K\langle T\rangle^{\times} \stackrel{\partial}{\longrightarrow}\left(K\langle X, Y\rangle^{\times}\right)^{\mathfrak{S}_{2}} \stackrel{\partial}{\longrightarrow} K\langle U, V, W\rangle^{\times}
$$

où

$$
\begin{aligned}
\forall f \in K\langle T\rangle^{\times}, \quad \partial f & =f(X+Y) f(X)^{-1} f(Y)^{-1} \\
\forall g \in K\langle X, Y\rangle^{\times}, \quad \partial g & =g(V, W) g(U+V, W)^{-1} g(U, V+W) g(U, V)^{-1} .
\end{aligned}
$$

Le $H^{2}$ de ce complexe est le même que celui du sous-complexe

$$
1+T \mathfrak{m}_{K}\langle T\rangle \stackrel{\partial}{\longrightarrow}\left(1+(X, Y) \mathfrak{m}_{K}\langle X, Y\rangle\right)^{\mathfrak{S}_{2}} \stackrel{\partial}{\longrightarrow} 1+(U, V, W) \mathfrak{m}_{K}\langle U, V, W\rangle .
$$


Regardons maintenant ce qu'il se passe lorsqu'on complète formellement le long de l'origine l'extension précédente de groupes analytiques.

Lemme 16. Le complexe

$$
1+T K \llbracket T \rrbracket \stackrel{\partial}{\longrightarrow}(1+(X, Y) K \llbracket X, Y \rrbracket)^{\mathfrak{S}_{2}} \stackrel{\partial}{\longrightarrow} 1+(U, V, W) K \llbracket U, V, W \rrbracket
$$

vérifie

$$
\begin{aligned}
& H^{1}=\{\exp (a T) \mid a \in K\} \\
& H^{2}=0 .
\end{aligned}
$$

Démonstration. Le calcul du $H^{1}$ est clair, il s'agit du calcul des morphismes de groupes formels $\operatorname{Hom}\left(\widehat{\mathbb{G}}_{a}, \widehat{\mathbb{G}}_{m}\right)$. La nullité du $H^{2}$ est équivalente à dire que toute extension de groupes formels de $\widehat{\mathbb{G}}_{a}$ par $\widehat{\mathbb{G}}_{m}$ est triviale ce qui est clair puisque car $(K)=0$ (on peut bien sûr le vérifier à la main en utilisant le calcul de cocyle de Lazard sur les gradués de la filtration $\left.\left(1+(X, Y)^{k} K \llbracket X, Y \rrbracket \stackrel{\partial}{\rightarrow} 1+(U, V, W)^{k} K \llbracket U, V, W \rrbracket\right)_{k \geq 1}\right)$.

On en déduit la proposition qui suit.

Proposition 23. Le groupe $\operatorname{Ext}^{1}\left(\mathbb{B}(0,1), \mathbb{G}_{m}^{a n}\right)$ est isomorphe au groupe

$$
\left\{f \in 1+T K \llbracket T \rrbracket \mid \partial f \in 1+\mathfrak{m}_{K}\langle X, Y\rangle\right\} /(1+T K\langle T\rangle) \cdot \exp (K . T) .
$$

Proposition 24. L'application $\operatorname{Ext}^{1}\left(\mathbb{B}(0,1), \mathbb{G}_{m}^{a n}\right) \stackrel{\times p}{\longrightarrow} \operatorname{Ext}^{1}\left(\mathbb{B}(0,1), \mathbb{G}_{m}^{a n}\right)$ est nulle.

Démonstration. Il faut montrer que si $f \in 1+T K \llbracket T \rrbracket$ vérifie

$$
f(X+Y) f(X)^{-1} f(Y)^{-1} \in 1+\mathfrak{m}_{K}\langle X, Y\rangle
$$

il existe alors $a \in K$ tel que $f^{p} \exp (a T) \in 1+\mathfrak{m}_{K}\langle T\rangle$. D'après le lemme 9.11 de [9] $a=-f^{\prime}(0)$ convient.

Corollaire 14. L'image du morphisme $\operatorname{Ext}^{1}\left(\mathbb{B}(0,1), \mu_{p^{n+1}}\right) \longrightarrow \operatorname{Ext}^{1}\left(\mathbb{B}(0,1), \mu_{p^{n}}\right)$ induit par le morphisme $\mu_{p^{n+1}} \stackrel{\times p}{\longrightarrow} \mu_{p^{n}}$ est contenue dans l'image de l'application bord de la suite de Kümmer

$$
\operatorname{Hom}\left(\mathbb{B}(0,1), \mathbb{G}_{m}^{a n}\right) \longrightarrow \operatorname{Ext}^{1}\left(\mathbb{B}(0,1), \mu_{p^{n}}\right) .
$$

Démonstration. Cela découle de l'existence d'un diagramme commutatif

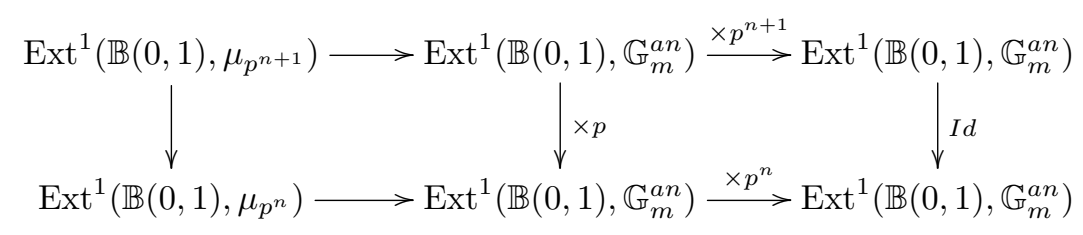

On vérifie facilement les deux lemmes qui suivent.

Lemme 17. Il y a un isomorphisme de groupes

$$
\begin{aligned}
& \stackrel{\circ}{\mathbb{B}}\left(0, p^{-\frac{1}{p-1}}\right)(K) \stackrel{\sim}{\longrightarrow} \operatorname{Hom}\left(\mathbb{B}(0,1), \mathbb{G}_{m}^{a n}\right) \\
& a \longmapsto \exp (a . T)
\end{aligned}
$$

Lemme 18. Soit $\varphi: \mathbb{R}_{>0} \stackrel{\sim}{\longrightarrow} \mathbb{R}_{>0}$ la fonction définie par

$$
\varphi(x)=\left\{\begin{array}{c}
1+x \text { si } x \geq \frac{1}{p-1} \\
p x \text { si } x \leq \frac{1}{p-1}
\end{array}\right.
$$

Pour $\epsilon \in\left|K^{\times}\right|$tel que $\epsilon<1$ notons $\mathbb{B}(1, \epsilon)^{1 / p^{n}}$ le sous-groupe affinö̈de de $\mathbb{G}_{m}^{\text {an }}$ image réciproque via le morphisme $x \mapsto x^{p^{n}}$ du sous-groupe $\mathbb{B}(1, \epsilon)$. Alors si $\lambda \in v\left(K^{\times}\right), \lambda>0$,

$$
\mathbb{B}\left(1, p^{\lambda}\right)^{1 / p^{n}}=\mu_{p^{n}} \cdot \mathbb{B}\left(1, p^{\varphi^{-n}(\lambda)}\right) .
$$


Théorème 3.1. Soit

$$
0 \longrightarrow \mu_{p^{n}} \longrightarrow G \longrightarrow \mathbb{B}(0,1) \longrightarrow 0
$$

une extension de groupes analytiques telle qu'il existe une autre extension ainsi qu'un diagramme

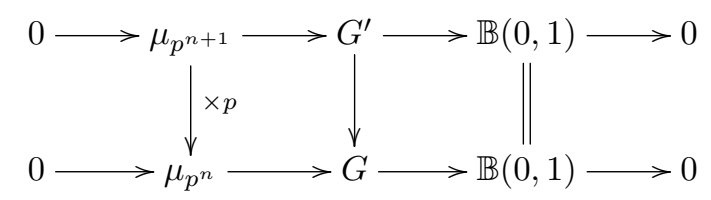

Il existe alors $a \in K^{\times}$tel que $v(a)>\frac{1}{p-1}$ et un diagramme

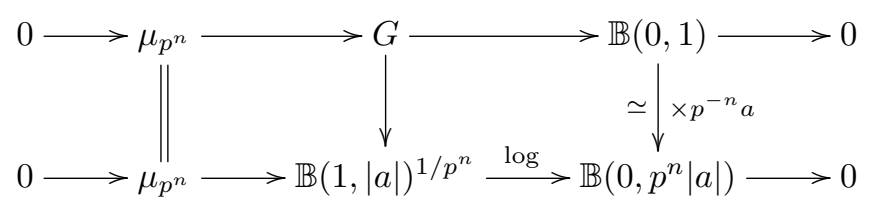

tel que l'extension du haut soit obtenue par image réciproque de celle du base. De plus deux telles extensions associées à a et $a^{\prime}$ sont isomorphes si et seulement si $v\left(a-a^{\prime}\right)>n+\frac{1}{p-1}$.

Démonstration. D'après le corollaire 14 une telle extension est classifiée par un élément de $\operatorname{Hom}\left(\mathbb{B}(0,1), \mathbb{G}_{m}^{a n}\right) / p^{n} \operatorname{Hom}\left(\mathbb{B}(0,1), \mathbb{G}_{m}^{a n}\right)$. Utilisant le lemme 17 on en déduit qu'elle est classifié par un élément de $\mathbb{B}\left(0, p^{-\frac{1}{p-1}}\right)(K) / p^{n} \mathbb{B}\left(0, p^{-\frac{1}{p-1}}\right)(K)$. On en déduit le théorème en retraçant les différentes identifications.

3.0.2. Le cas des groupes de dimension 1 et de hauteur 1 . On note $C$ un corps algébriquement clos valué complet pour une valuation de rang 1.

Théorème 3.2. Soit $G$ un $C$-groupe analytique rigide de type p-divisible de hauteur 1 et de dimension 1 . Alors soit $G \simeq \widehat{\mathbb{G}}_{m}^{\text {rig }}$ soit $G \simeq \mathbb{Q}_{p} / \mathbb{Z}_{p} \times \mathbb{G}_{a}^{\text {rig }}$.

Démonstration. Puisque $C$ est algébriquement clos on peut fixer un isomorphisme $G\left[p^{\infty}\right] \simeq \mu_{p} \infty$. Soit $U \subset G$ un sous-groupe affinoïde connexe tel que $\log _{G \mid U}$ soit un isomorphisme sur son image. Fixons un isomorphisme Lie $G \stackrel{\sim}{\longrightarrow} C$ tel qu'il induise un isomorphisme $\log _{G}(U) \stackrel{\sim}{\longrightarrow} \mathbb{B}(0,1)$ (les sous-groupes affinoïdes connexes de $\mathbb{G}_{a}^{r i g}$ sont les boules fermées). Notons pour tout entier $n \geq 0$, $U_{n}=p^{-n} U$. Grâce aux deux identifications faites il s'insère dans une extension

$$
0 \longrightarrow \mu_{p^{n}} \longrightarrow U_{n} \stackrel{\log }{\longrightarrow} \mathbb{B}\left(0, p^{n}\right) \longrightarrow 0
$$

Il y a de plus un diagramme commutatif

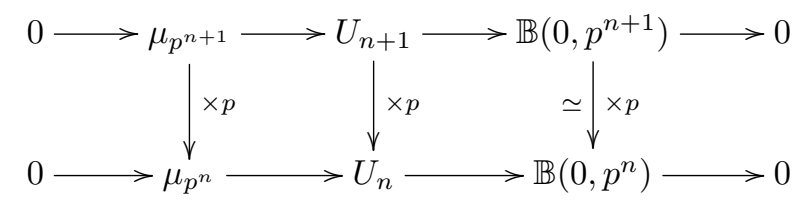

D'après le théorème 3.1 l'extension précédente associée à $U_{n}$ est donc classifiée par un élément $\xi_{n} \in \stackrel{\circ}{B}\left(0, p^{-\frac{1}{p-1}}\right)(C) / p^{n} \mathbb{B}\left(0, p^{-\frac{1}{p-1}}\right)(C)$. Considérons le morphisme d'extensions

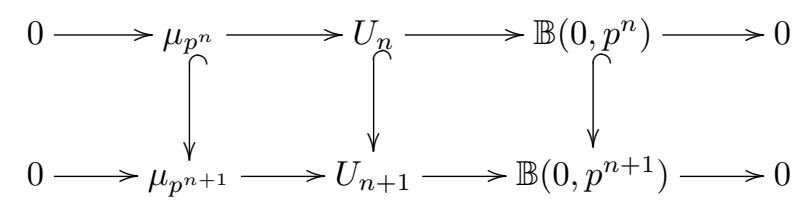

on vérifie qu'il implique que $\xi_{n} \equiv \xi_{n+1} \bmod p^{n} \stackrel{\circ}{\mathbb{B}}\left(0, p^{-\frac{1}{p-1}}\right)(C)$. L'extension associée à $G=\underset{n \geq 0}{\lim } \underset{n}{U_{n}}$

$$
0 \longrightarrow \mu_{p^{\infty}} \longrightarrow G \longrightarrow \mathbb{G}_{a}^{r i g} \longrightarrow 0
$$


est donc donnée par un élément

$$
\xi \in \lim _{\substack{\longleftarrow \geq 0}} \stackrel{\circ}{\mathbb{B}}\left(0, p^{-\frac{1}{p-1}}\right)(C) / p^{n} \stackrel{\circ}{\mathbb{B}}\left(0, p^{-\frac{1}{p-1}}\right)(C)=\stackrel{\circ}{\mathbb{B}}\left(0, p^{-\frac{1}{p-1}}\right)(C)
$$

de telle manière que si $\xi \neq 0$ il y ait des isomorphismes compatibles lorsque $n$ varie

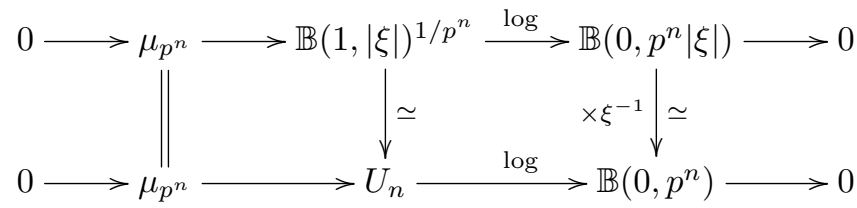

Passant à la limite sur $n$ on en déduit que si $\xi \neq 0$ il existe un isomorphisme

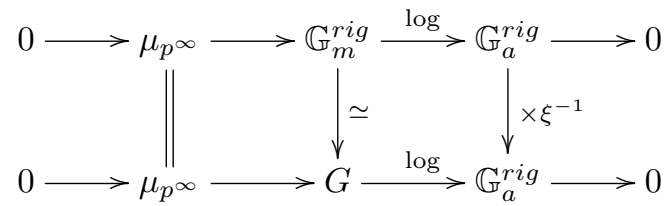

Si $\xi=0$ il est de même facile de vérifier que $G \simeq \mathbb{G}_{a}^{r i g} \times \mu_{p^{\infty}}$.

\subsubsection{Le cas général.}

Théorème 3.3. Soit $G$ un $C$-groupe analytique rigide de type $p$-divisible. Notons $\Lambda=T_{p}\left(G\left[p^{\infty}\right]\right)$. Il existe alors un unique morphisme $C$-linéaire

$$
f: \text { Lie } G \longrightarrow \Lambda \otimes_{\mathbb{Z}_{p}} C(-1)
$$

et un diagramme

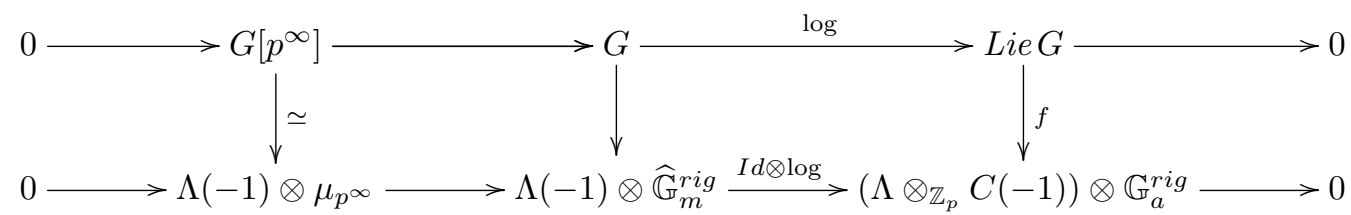

tel que l'extension du haut soit l'image réciproque de celle du bas via $f$.

La catégorie $\mathcal{R}_{C}$ des $C$-groupes analytiques rigides de type p-divisible est équivalente à celle des triplets $(V, \Lambda, f)$ où $V$ est un $C$-espace vectoriel de dimension finie, $\Lambda$ un $\mathbb{Z}_{p}$-module de type fini et $f: V \longrightarrow \Lambda \otimes_{\mathbb{Z}_{p}} C(-1)$ un morphisme $C$-linéaire.

Démonstration. Si $W$ est un $C$-espace vectoriel de dimension finie et $\Lambda$ un $\mathbb{Z}_{p}$-module de type fini il y a un isomorphisme canonique

$$
\begin{aligned}
\operatorname{Ext}_{\text {loc.sc. }}\left(W \otimes \mathbb{G}_{a}^{r i g}, \Lambda(-1) \otimes_{\mathbb{Z}_{p}} \mu_{p^{\infty}}\right) & \stackrel{\sim}{\longrightarrow} W^{*} \otimes_{C} \operatorname{Ext}_{\text {loc.sc. }}\left(\mathbb{G}_{a}^{r i g}, \mu_{p^{\infty}}\right) \otimes_{\mathbb{Z}_{p}} \Lambda(-1) \\
& =\operatorname{Hom}_{C}(W, \Lambda(-1) \otimes C) \otimes_{C} \operatorname{Ext}_{\text {loc.sc. }}\left(\mathbb{G}_{a}^{r i g}, \mu_{p^{\infty}}\right) .
\end{aligned}
$$

D'après le théorème 3.2, $\operatorname{Ext}_{\text {loc.sc. }}\left(\mathbb{G}_{a}^{\text {rig }}, \mu_{p^{\infty}}\right)$ est un $C$-espace vectoriel de dimension 1 de base la classe de l'extension associée à $\mathbb{G}_{m}^{r i g}$. D'où le résultat.

Corollaire 15. Tout $C$-groupe analytique rigide connexe de type p-divisible est isomorphe en tant qu'espace rigide au produit d'un espace affine par une sous-variété Zariski fermée d'une boule ouverte.

Corollaire 16. La catégorie $\mathcal{R}_{C} \otimes \mathbb{Q}$ des $C$-groupes analytiques rigides de type p-divisible à isogénie près est abélienne. Elle est équivalente à la catégorie formée des triplets $(W, V, f)$ où $W$ est un $C$-espace vectoriel de dimension finie, $V$ un $\mathbb{Q}_{p}$-espace vectoriel de dimension finie et $f: W \longrightarrow$ $V_{C}(-1)$ une application $C$-linéaire. 
3.0.4. Le cas des groupes analytiques rigides associés aux groupes formels p-divisibles.

Lemme 19. Soient $G_{1}$ et $G_{2}$ deux groupes analytiques rigides de type $p$-divisible sur $C$. Soit $\Lambda$ un $\mathbb{Z}_{p}$-module libre de type fini. Il y a alors un isomorphisme naturel

$$
\operatorname{Hom}\left(G_{1}, \Lambda^{*} \otimes_{\mathbb{Z}_{p}} G_{2}\right) \stackrel{\sim}{\longrightarrow} \operatorname{Hom}\left(\Lambda \otimes_{\mathbb{Z}_{p}} G_{1}, G_{2}\right) .
$$

Démonstration. Il y a une application naturelle de morphismes de foncteurs en $\mathbb{Z}_{p}$-modules sur les $C$-algèbres affinoïdes

$$
\operatorname{Hom}\left(G_{1}, \Lambda^{*} \otimes_{\mathbb{Z}_{p}} G_{2}\right) \stackrel{\Lambda \otimes-}{\longrightarrow} \operatorname{Hom}\left(\Lambda \otimes_{\mathbb{Z}_{p}} G_{1}, \Lambda \otimes_{\mathbb{Z}_{p}} \Lambda^{*} \otimes_{\mathbb{Z}_{p}} G_{2}\right) \longrightarrow \operatorname{Hom}\left(\Lambda \otimes_{\mathbb{Z}_{p}} G_{1}, G_{2}\right)
$$

où la seconde application est donnée par le morphisme $\mathbb{Z}_{p}$-linéaire de contraction des tenseurs $\Lambda \otimes_{\mathbb{Z}_{p}} \Lambda^{*} \longrightarrow \mathbb{Z}_{p}$. On vérifie aussitôt en fixant une base de $\Lambda$ que c'est une bijection.

Théorème 3.4. Soit $\mathcal{G}$ un groupe formel p-divisible sur $\operatorname{Spf}\left(\mathcal{O}_{C}\right)$. Soit $(V, \Lambda, f)$ le triplet associé $\grave{a} \mathcal{G}^{\text {rig }}$ par le théorème 3.3. On a alors

$-V=\operatorname{Lie} \mathcal{G}\left[\frac{1}{p}\right]$

$-\Lambda=T_{p}(\mathcal{G})$

- $f:$ Lie $G\left[\frac{1}{p}\right] \longrightarrow V_{p}(\mathcal{G}) \otimes_{\mathbb{Q}_{p}} C(-1)$ est la transposée de l'application de Hodge-Tate de $\mathcal{G}^{D}$

$$
V_{p}(\mathcal{G})^{*} \otimes C(1)=V_{p}\left(\mathcal{G}^{D}\right) \otimes C \longrightarrow \omega_{\mathcal{G}}\left[\frac{1}{p}\right]
$$

En particulier le groupe analytique rigide $\mathcal{G}^{\text {rig }}$ détermine complètement l'application de Hodge-Tate de $\mathcal{G}^{D}$.

Démonstration. Rappelons que l'application de Hodge-Tate de $\mathcal{G}^{D}$ est donnée par

$$
\begin{aligned}
\alpha_{\mathcal{G}^{D}}: T_{p}\left(\mathcal{G}^{D}\right)=\operatorname{Hom}\left(\mathbb{Q}_{p} / \mathbb{Z}_{p}, \mathcal{G}^{D}\right)=\operatorname{Hom}\left(\mathcal{G}, \mu_{p^{\infty}}\right) & \longrightarrow \omega_{\mathcal{G}} \\
f & \longmapsto f^{*} \frac{d T}{T}
\end{aligned}
$$

Il y a un morphisme naturel de groupes analytiques rigides

$$
T_{p}\left(\mathcal{G}^{D}\right) \otimes_{\mathbb{Z}_{p}} \mathcal{G}^{r i g}=\operatorname{Hom}\left(\mathcal{G}, \widehat{\mathbb{G}}_{m}\right) \otimes_{\mathbb{Z}_{p}} \mathcal{G}^{\text {rig }} \longrightarrow \widehat{\mathbb{G}}_{m}^{r i g}
$$

qui d'après le lemme 19 induit un morphisme

$$
f: \mathcal{G}^{r i g} \longrightarrow T_{p}\left(\mathcal{G}^{D}\right)^{*} \otimes_{\mathbb{Z}_{p}} \widehat{\mathbb{G}}_{m}^{r i g} .
$$

Il suffit alors de vérifier que le diagramme

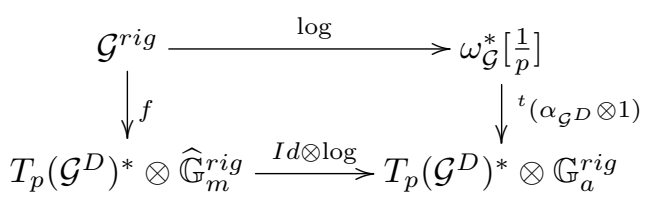

commute.

4. Classification des groupes analytiques Rigides De type $p$-Divisible Sur un CORPS QUELCONQUE

4.1. Classification des groupes analytiques rigides de type $p$-divisibles munis d'une donnée de descente. Soit $K$ un corps valué complet pour une valuation de rang 1 . Soit $\bar{K}$ une clôture algébrique de $K$. On note $C=\widehat{\bar{K}}$.

Définition 7. Si $G$ est un $C$-groupe analytique rigide de type p-divisible une donnée de descente de $C$ à $K$ sur $G$ est la donnée d'isomorphismes pour $\sigma \in \operatorname{Gal}(\bar{K} \mid K)$

$$
u_{\sigma}: G^{(\sigma)} \stackrel{\sim}{\longrightarrow} G
$$

vérifiant la relation de cocycle pour $\sigma, \tau \in \operatorname{Gal}(\bar{K} \mid K)$

$$
u_{\tau} \circ u_{\sigma}^{(\tau)}=u_{\tau \sigma} .
$$


Un morphisme $\left(G,\left(u_{\sigma}\right)_{\sigma}\right) \longrightarrow\left(G^{\prime},\left(v_{\sigma}\right)_{\sigma}\right)$ de $C$-groupes analytiques rigides munis de données de descentes de $C$ à $K$ est un morphisme de groupes $f: G \longrightarrow G^{\prime}$ tel que pour tout $\sigma \in G a l(\bar{K} \mid K)$ on ait $f \circ u_{\sigma}=v_{\sigma} \circ f^{(\sigma)}$.

Exemple 8. Si $W$ est un $C$-espace vectoriel une donnée de descente de $C$ à $K$ sur $W \otimes \mathbb{G}_{a}^{\text {rig }}$ est équivalente à la donnée d'une action $C$-semi-linéaire de $\operatorname{Gal}(\bar{K} \mid K)$ sur $W$ par automorphismes C-linéaires.

Exemple 9. Si $\Gamma$ est un groupe p-divisible sur $C$ une donnée de descente de $C$ à $K$ sur $\Gamma$ est équivalente à la donnée d'une action linéaire de $\operatorname{Gal}(\bar{K} \mid K)$ sur $T_{p}(\Gamma)$.

On remarquera que si $G$ est un $C$-groupes analytique rigide muni d'une donnée de descente de $C$ à $K$ alors $G\left[p^{\infty}\right]$ et Lie $G \otimes \mathbb{G}_{a}^{r i g}$ sont alors munis d'une telle donnée de descente. En particulier $T_{p}\left(G\left[p^{\infty}\right]\right)$ est muni d'une action linéaire de $\operatorname{Gal}(\bar{K} \mid K)$ et Lie $G$ d'une action semi-linéaire.

Définition 8. Si $G$ est un $C$-groupe analytique rigide de type p-divisible une donnée de descente continue de $C$ à $K$ sur $G$ est une donnée de descente de $C$ à $K$ sur $G$ telle que l'action de $\operatorname{Gal}(\bar{K} \mid K)$ induite sur l'espace topologique $G(C)$ soit continue. On note $\mathcal{R}_{C / K}$ la catégorie des $C$-groupes analytiques rigides de type p-divisible munis d'une donnée de descente continue de $C$ à $K$.

Lemme 20. Soit $G$ un $C$-groupe analytique rigide. Une donnée de descente de $C$ à $K$ sur $G$ est continue si et seulement l'action semi-linéaire de $G a l(\bar{K} \mid K)$ induite sur Lie $G$ et l'action linéaire induite sur $T_{p}\left(G\left[p^{\infty}\right]\right)$ sont continues.

Démonstration. Si une donnée de descente sur $G$ est continue l'action de $\operatorname{Gal}(\bar{K} \mid K)$ induites sur $G\left[p^{\infty}\right](C)$ l'est également. Or $G\left[p^{\infty}\right](C)$ est discret dans $G(C)$ et l'action de $\operatorname{Gal}(\bar{K} \mid K)$ sur $G\left[p^{\infty}\right](C)$ est donc discrète c'est à dire l'action sur $T_{p}\left(G\left[p^{\infty}\right]\right)$ est continue. Pour une donnée de descente fixée sur $G$ le morphisme $\log : G(C) \longrightarrow$ Lie $G$ est $\operatorname{Gal}(\bar{K} \mid K)$-équivariant surjectif. Si cette donnée de descente est continue on en déduit que l'action induite sur Lie $G$ est continue.

Réciproquement soit $G$ muni d'une donnée de descente qui induise des action continues sur Lie $G$ et $T_{p}\left(G\left[p^{\infty}\right]\right)$. D'après le théorème 3.3 le groupe $G$ est déterminé par un morphisme $C$-linéaire $f:$ Lie $G \longrightarrow T_{p}\left(G\left[p^{\infty}\right]\right) \otimes C(-1)$. La donnée de descente sur $G$ fournit des action de $\operatorname{Gal}(\bar{K} \mid K)$ sur $W=\operatorname{Lie} G$ et $\Lambda=T_{p}\left(G\left[p^{\infty}\right]\right)$. De plus le morphisme $f$ est $\operatorname{Gal}(\bar{K} \mid K)$-équivariant. Alors,

$$
G(C)=\left(\left(1+\mathfrak{m}_{C}\right) \otimes_{\mathbb{Z}_{p}} \Lambda(-1)\right) \underset{\Lambda(-1) \otimes C}{\times} W
$$

comme espace topologique muni d'une action de $\operatorname{Gal}(\bar{K} \mid K)$ où $\mathfrak{m}_{C}$ désigne l'idéal maximal de $\mathcal{O}_{C}$. Le lemme s'en déduit.

Proposition 25. La catégorie $\mathcal{R}_{C / K}$ est équivalente à la catégorie des triplets $(W, \Lambda, f)$ où $W$ est un $C$-espace vectoriel muni d'une action semi-linéaire continue de $G a l(\bar{K} \mid K), \Lambda$ est un $\mathbb{Z}_{p}$-module libre de type fini muni d'une action linéaire continue de $G a l(\bar{K} \mid K)$ et $f: W \longrightarrow \Lambda \otimes_{\mathbb{Z}_{p}} C(-1)$ est un morphisme $C$-linéaire compatible à l'action de $\operatorname{Gal}(\bar{K} \mid K)$.

Démonstration. Il s'agit d'une application du lemme 20 et du théorème 3.3.

\subsection{Classification des $K$-groupes analytiques rigides de type $p$-divisible.}

Proposition 26. Le foncteur d'extension des scalaires qui va de la catégorie des $K$-espaces rigides analytiques vers la catégorie des $C$-espaces rigides analytiques munis d'une donnée de descente de $C$ à $K$ est pleinement fidèle.

Démonstration. Il suffit de montrer que si $A$ et $\mathrm{B}$ sont deux $K$-algèbres affinoïdes alors tout morphisme de $C$-algèbres

$$
A \hat{\otimes}_{K} C \longrightarrow \mathrm{B} \hat{\otimes}_{K} C
$$

qui commute à l'action de galois est obtenu par extension des scalaires d'un morphisme de $K$ algèbres de $A$ vers $\mathrm{B}$. Il suffit pour cela de montrer que

$$
\mathrm{B}=\left(\mathrm{B} \hat{\otimes}_{K} C\right)^{\operatorname{Gal}(\bar{K} \mid K)} .
$$


Mais d'après le théorème 2.8.2/2 de [6] il existe un homéomorphisme de $K$-espaces de Banach

$$
c(K) \stackrel{\sim}{\longrightarrow} \mathrm{B}
$$

où

$$
c(K)=\left\{\left(x_{i}\right)_{i \in \mathbb{N}} \mid x_{i} \in K, \lim _{i \rightarrow+\infty} x_{i}=0\right\}
$$

muni de la norme $\left\|\left(x_{i}\right)_{i}\right\|=\sup _{i \in \mathbb{N}}\left|x_{i}\right|$. Cela induit un homéomorphisme $\operatorname{Gal}(\bar{K} \mid K)$-équivariant

$$
c(C)=c(K) \hat{\otimes}_{K} C \stackrel{\sim}{\longrightarrow} \mathrm{B} \hat{\otimes}_{K} C .
$$

D'après le théorème de Ax-Sen-Tate $([2]) C^{\mathrm{Gal}(\bar{K} \mid K)}=K$ et donc

$$
c(C)^{\operatorname{Gal}(\bar{K} \mid K)}=c(K)
$$

ce qui implique le résultat.

Théorème 4.1. Le foncteur d'extension des scalaires $\mathcal{R}_{K} \longrightarrow \mathcal{R}_{C / K}$ induit une équivalence de catégories entre la catégorie des $K$-groupes analytiques rigides de type p-divisible et la catégorie des $C$-groupes analytiques rigides de type p-divisibles munis d'une donnée de descente continue de $C$ à $K$ tels que si $W$ désigne leur algèbre de Lie, il existe $W^{\prime}$ un $K$-espace vectoriel de dimension finie et un isomorphisme $\operatorname{Gal}(\bar{K} \mid K)$-équivariant $W^{\prime} \otimes_{K} C \stackrel{\sim}{\longrightarrow} W$.

Démonstration. La pleine fidélité du foncteur résulte de la proposition 26 . Reste à démontrer la surjectivité essentielle. Soient $\Lambda$ un $\mathbb{Z}_{p}$-module libre de type fini muni d'une action continue de $\operatorname{Gal}(\bar{K} \mid K), W^{\prime}$ un $K$-espace vectoriel de dimension finie et $f: W^{\prime} \otimes_{K} C \longrightarrow \Lambda(-1) \otimes_{\mathbb{Z}_{p}} C$ un morphisme $C$-linéaire compatible à l'action de $\operatorname{Gal}(\bar{K} \mid K)$. Le $C$-groupe analytique rigide de type $p$-divisible

$$
G=\Lambda(-1) \otimes_{\mathbb{Z}_{p}} \widehat{\mathbb{G}}_{m}^{r i g} \underset{\Lambda(-1) \otimes_{\mathbb{Z}_{p}} \mathbb{G}_{a}^{r i g}}{\times}\left(W^{\prime} \otimes_{K} C\right) \otimes \mathbb{G}_{a}^{r i g}
$$

est muni d'une donnée de descente de $C$ à $K$ dont il s'agit de voir qu'elle est effective. Soit $\mathbb{B} \subset W^{\prime} \otimes \mathbb{G}_{a}^{r i g}$ un sous-groupe affinoïde qui après choix d'une base de $W^{\prime}$ est une boule de rayon suffisamment petit tel que $\log _{G}: G \longrightarrow W_{C}^{\prime} \otimes \mathbb{G}_{a}^{r i g}$ possède une section au dessus de $\mathbb{B} \hat{\otimes}_{K} C$. Soit $U=\left(\log _{G}^{-1}(\mathbb{B} \hat{\otimes} C)\right)^{0}$, un sous-groupe affinoïde de $G$ tel que $\log _{G}: U \stackrel{\sim}{\longrightarrow} \mathbb{B} \hat{\otimes} C$. Pour un entier $n \geq 0$ soit $U_{n}=p^{-n} U$. On vérifie que la donnée de descente sur $G$ induit une donnée de descente de $C$ à $K$ sur le groupe affinoïde $U_{n}$. D'après le théorème 3.1 l'extension de $C$-groupes analytiques

$$
0 \longrightarrow \Lambda(-1) \otimes_{\mathbb{Z}_{p}} \mu_{p^{n}} \longrightarrow U_{n} \longrightarrow \mathbb{B} \hat{\otimes} C \longrightarrow 0
$$

est donnée par un élément

$$
\xi \in \operatorname{Hom}\left(\mathbb{B} \hat{\otimes} C, \mathbb{G}_{m}^{r i g}\right) \otimes_{\mathbb{Z}_{p}} \Lambda(-1) / p^{n} \Lambda(-1)=\operatorname{Hom}\left(\mathbb{B} \hat{\otimes} C, \stackrel{\circ}{\mathbb{B}}\left(0, p^{-\frac{1}{p-1}}\right)\right) \otimes_{\mathbb{Z}_{p}} \Lambda(-1) / p^{n} \Lambda(-1) .
$$

Il est facile de vérifier que ce groupe s'identifie à

$$
\underset{\substack{L \mid K \text { finie } \\ L \subset C}}{\lim } \operatorname{Hom}\left(\mathbb{B} \hat{\otimes} L, \mathbb{G}_{m}^{r i g}{ }^{r}\right) \otimes_{\mathbb{Z}_{p}} \Lambda(-1) / p^{n} \Lambda(-1)
$$

Utilisant le fait que l'action de $\operatorname{Gal}(\bar{K} \mid K) \operatorname{sur} \Lambda(-1) / p^{n} \Lambda(-1)$ est discrète on en déduit qu'il existe une extension galoisienne $L \mid K$ finie telle $\operatorname{Gal}(\bar{K} \mid L)$ agisse trivialement sur $\Lambda(-1) / p^{n} \Lambda(-1)$,

$$
\xi \in \operatorname{Hom}\left(\mathbb{B} \hat{\otimes} L, \mathbb{G}_{m}^{r i g} / L\right) \otimes_{\mathbb{Z}_{p}} \Lambda(-1) / p^{n} \Lambda(-1)
$$

et $\xi$ est $\operatorname{Gal}(L \mid K)$-invariante pour l'action de $\operatorname{Gal}(L \mid K)$ déduite de celle sur $\mathbb{B} \hat{\otimes} L$ et sur $\Lambda(-1) / p^{n} \Lambda(-1)$. Cela définit alors un $L$-groupe analytique rigide affinoïde $V_{n}$ muni d'une donnée de descente de $L$ à $K$ et tel que $V_{n} \hat{\otimes}_{L} C \simeq U_{n}$ comme groupe analytique rigide muni d'une donnée de descente de $C$ à $K$. Puisque l'extension $L \mid K$ est finie il existe un $K$-groupe analytique rigide affinoïde $W_{n}$ tel que $U_{n} \simeq W_{n} \hat{\otimes}_{K} C$ comme groupe muni d'une donnée de descente.

La proposition 26 montre alors que les groupes $U_{n}$ se recollent lorsque $n$ varie pour former un $K$-groupe analytique rigide de type $p$-divisible qui redonne $G$ muni de sa donnée de descente après extension des scalaires à $C$. 
Remarque 8. Dans le théorème précédent l'espace vectoriel $W^{\prime}$ est déterminé de façon fonctorielle par $W$ puisque d'après le théorème d'Ax-Sen-Tate on peut prendre $W^{\prime}=W^{G a l(\bar{K} \mid K)}$.

Corollaire 17. La catégorie $\mathcal{R}_{K}$ est équivalente à celle des triplets $(W, \Lambda, f)$ où $W$ est un $K$ espace vectoriel de dimension finie, $\Lambda$ un $\mathbb{Z}_{p}$-module libre de type fini muni d'une action continue de $G a l(\bar{K} \mid K)$ et $f: W \longrightarrow \Lambda \otimes_{\mathbb{Z}_{p}} C(-1)$ est $C$-linéaire galois équivariant. Si $G \in \mathcal{R}_{K}$ le triplet associé $(W, \Lambda, f)$ est tel que $W=\operatorname{Lie} G, \Lambda=T_{p}\left(G\left[p^{\infty}\right]\right)$. Si de plus $\mathcal{G}$ est un groupe formel p-divisible sur $\operatorname{Spf}\left(\mathcal{O}_{K}\right)$ alors $W=$ Lie $\mathcal{G}\left[\frac{1}{p}\right], \Lambda=T_{p}(\mathcal{G})$ et $f$ est donné par la transposée de l'application de Hodge-Tate de $\mathcal{G}^{D}$.

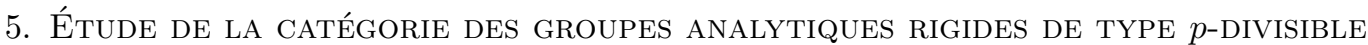

Soit $K$ un corps valué complet pour une valuation de rang 1 extension de $\mathbb{Q}_{p}$. Soit $\bar{K}$ une clôture algébrique de $K$ et $C=\widehat{\bar{K}}$.

5.1. Composantes connexes et facteurs additifs. Des corollaires 9 et 10 on déduit la proposition qui suit.

Proposition 27. Tout $C$-groupe analytique rigide de type p-divisible est isomorphe à un groupe de la forme

$$
\left(\mathbb{Q}_{p} / \mathbb{Z}_{p}\right)^{a} \oplus\left(\mathbb{G}_{a}^{r i g}\right)^{b} \oplus G
$$

où $G$ est un groupe analytique rigide de type p-divisible connexe sans facteur direct additif.

Proposition 28. Soit $G$ un $C$-groupe analytique rigide de type p-divisible et $f:$ Lie $G \longrightarrow$ $V_{p}\left(G\left[p^{\infty}\right]\right) \otimes_{\mathbb{Q}_{p}} C(-1)$ le morphisme associé par le théorème 3.3.

(1) La hauteur du groupe p-divisible $\pi_{0}(G)$ est égale à la dimension sur $\mathbb{Q}_{p} d u$ noyau de l'application composée

$$
V_{p}\left(G\left[p^{\infty}\right]\right)^{*} \hookrightarrow V_{p}\left(G\left[p^{\infty}\right]\right)_{C}^{*} \otimes C(1) \stackrel{{ }^{t} f}{\longrightarrow} \operatorname{Lie}(G)^{*} .
$$

En particulier $G$ est connexe si et seulement si cette application est injective.

(2) La dimension de Hom $\left(\mathbb{G}_{a}^{r i g}, G\right)$ est égale la dimension du noyau de $f$. En particulier $G$ est sans facteur direct additif si et seulement si $f$ est injective.

Démonstration. La hauteur de $\pi_{0}(G)$ est le rang du $\mathbb{Z}_{p}$-module $\operatorname{Hom}\left(G, \mathbb{Q}_{p} / \mathbb{Z}_{p}\right)$. Mais $\operatorname{Hom}\left(G, \mathbb{Q}_{p} / \mathbb{Z}_{p}\right) \otimes$ $\mathbb{Q}_{p}=\operatorname{Hom}\left(\left(\operatorname{Lie} G, V_{p}\left(G\left[p^{\infty}\right]\right), f\right),\left(0, \mathbb{Q}_{p}, 0\right)\right)$.

De même $\operatorname{Hom}\left(\mathbb{G}_{a}^{r i g}, G\right)=\operatorname{Hom}\left((C, 0,0),\left(\operatorname{Lie} G, V_{p}\left(G\left[p^{\infty}\right]\right), f\right)\right)$.

Corollaire 18. Soit $G$ un groupe analytique rigide de type p-divisible sans facteur additif. Alors

$$
\operatorname{dim} G \leq \operatorname{ht} G
$$

\subsection{Foncteurs fibres génériques.}

Définition 9. On note $\operatorname{pdiv}_{\mathcal{O}_{K}}^{f}$ la catégorie des groupes formels p-divisibles sur $\operatorname{Spf}\left(\mathcal{O}_{K}\right)$ et $\operatorname{pdiv}_{\mathcal{O}_{K}}^{f} \otimes \mathbb{Q}$ pour la catégorie des groupes formels p-divisibles sur $\operatorname{Spf}\left(\mathcal{O}_{K}\right)$ à isogénies près.

Définition 10. On note $\mathcal{R}_{K}^{a}$ la catégorie des $K$-groupes analytiques rigides de type p-divisible $G$ tels que $G \hat{\otimes} C$ soit sans facteur direct additif et $\mathcal{R}_{K}^{a} \otimes \mathbb{Q}$ la catégorie associée à isogénies près.

La catégorie $\mathcal{R}_{K} \otimes \mathbb{Q}$ est abélienne $\mathbb{Q}_{p}$-linéaire, équivalente à celle des triplets $(W, V, f)$ où $W$ est un $K$-espace vectoriel de dimension finie, $V$ est un $\mathbb{Q}_{p}$-espace vectoriel de dimension finie muni d'une action linéaire continue de $\operatorname{Gal}(\bar{K} \mid K)$ et $f: W \otimes_{K} C \longrightarrow V \otimes_{\mathbb{Q}_{p}} C(-1)$ est $C$-linéaire compatible à l'action de $\operatorname{Gal}(\bar{K} \mid K)$. La sous-catégorie $\mathcal{R}^{a} \otimes \mathbb{Q}$ correspond aux $(W, V, f)$ tels que $f$ soit injective.

Lemme 21. Si $H$ est un groupe formel p-divisible sur $\operatorname{Spf}\left(\mathcal{O}_{C}\right)$ alors $H^{\text {rig }}$ est sans facteur direct additif. 
Démonstration. On doit montrer que $\operatorname{Hom}\left(\mathbb{G}_{a}^{\text {rig }}, H^{\text {rig }}\right)=0$. Comme espace rigide $H^{\text {rig }} \simeq$ $\stackrel{\circ}{\mathbb{B}}^{d}(0,1)$ pour un entier $d$. Le lemme résulte donc du fait que toute fonction holomorphe bornée sur $\left(\mathbb{A}^{1}\right)^{\text {rig }}$ est constante.

Remarque 9. Le lemme 21 précédent est équivalent à l'assertion que pour tout groupe formel p-divisible $H$ sur $\mathcal{O}_{C}$ la transposée de l'application de Hodge-Tate de $H^{D}$ est injective. Cela est équivalent à la surjectivité de l'application de Hodge-Tate $V_{p}(H) \otimes C \longrightarrow \omega_{H^{D}} \otimes C$ pour tout groupe p-divisible $H$ sur $\mathcal{O}_{C}$. Ce fait n'est pas nouveau mais la démonstration précédente en donne une explication purement géométrique.

La catégorie des groupes formels $p$-divisibles $\operatorname{sur} \operatorname{Spf}\left(\mathcal{O}_{K}\right)$ est équivalente à celle des groupes $p$-divisibles sur $\operatorname{Spec}\left(\mathcal{O}_{K}\right)$ dont la fibre spéciale est formelle ([15], lemme 4.16). Il y a donc deux foncteurs fibres génériques ainsi qu'un diagramme commutatif

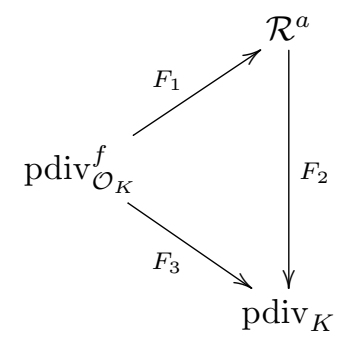

où si $H$ est un groupe formel $p$-divisible sur $\operatorname{Spf}\left(\mathcal{O}_{K}\right)$ on a $F_{1}(H)=H^{r i g}$ et $F_{3}(H)=\underset{n \geq 1}{\lim _{n \geq}}\left(H\left[p^{n}\right] \otimes K\right)$. De plus $F_{2}(G)=G\left[p^{\infty}\right]$.

Lemme 22. Soient $\mathfrak{X}_{1}$ et $\mathfrak{X}_{2}$ deux $\operatorname{Spf}\left(\mathcal{O}_{K}\right)$-schémas formels tels que pour $i=1,2$ il existe un entier $d_{i}$ tel que $\mathfrak{X}_{i} \simeq \operatorname{Spf}\left(\mathcal{O}_{K}\left(\llbracket x_{1}, \ldots, x_{d_{i}} \rrbracket\right)\right.$, l'anneau $\mathcal{O}_{K}\left(\llbracket x_{1}, \ldots, x_{d_{i}} \rrbracket\right.$ étant muni de la topologie $\left(p, x_{1}, \ldots, x_{d_{i}}\right)$-adique. L'application naturelle

$$
\operatorname{Hom}\left(\mathfrak{X}_{1}, \mathfrak{X}_{2}\right) \longrightarrow \operatorname{Hom}\left(\mathfrak{X}_{1}^{\text {rig }}, \mathfrak{X}_{2}^{\text {rig }}\right)
$$

est alors une bijection.

Démonstration. Notons pour $i=1,2, \mathfrak{X}_{i}=\operatorname{Spf}\left(A_{i}\right)$. Il y a des injections naturelles

$$
A_{i}=\Gamma\left(\mathfrak{X}_{i}, \mathcal{O}_{\mathfrak{X}_{i}}\right) \hookrightarrow \Gamma\left(\mathfrak{X}_{i}, \mathcal{O}_{\mathfrak{X}_{i}}\right)\left[\frac{1}{p}\right] \hookrightarrow \Gamma\left(\mathfrak{X}_{i}^{\text {rig }}, \mathcal{O}_{\mathfrak{X}_{i}^{\text {rig }}}\right)
$$

telles que

$$
A_{i}=\Gamma\left(\mathfrak{X}_{i}^{r i g}, \mathcal{O}_{\mathfrak{X}_{i}^{r i g}}^{0}\right):=\left\{f \in \Gamma\left(\mathfrak{X}_{i}^{r i g}, \mathcal{O}_{\mathfrak{X}_{i}^{r i g}} \mid\|f\|_{\infty} \leq 1\right\}\right.
$$

De plus dans $A_{i}$

$$
\mathfrak{m}_{A_{i}}=\sqrt{\left(p, x_{1}, \ldots, x_{d_{i}}\right)}=\left\{f \in \Gamma\left(\mathfrak{X}_{i}^{r i g}, \mathcal{O}_{\mathfrak{X}_{i}^{r i g}}^{0}\right)\left|\forall x \in \mathfrak{X}^{\text {rig }},\right| f(x) \mid<1\right\}
$$

où $\mathfrak{m}_{A_{i}}$ est l'idéal maximal de $A_{i}$. De cela on déduit qu'il y a une application

$$
\operatorname{Hom}\left(\mathfrak{X}_{1}^{\text {rig }}, \mathfrak{X}_{2}^{\text {rig }}\right) \longrightarrow \operatorname{Hom}\left(\mathfrak{X}_{1}, \mathfrak{X}_{2}\right)
$$

qui au morphisme $f$ associe le morphisme de schémas formels associé à $f^{*}: \Gamma\left(\mathfrak{X}_{2}^{\text {rig }}, \mathcal{O}_{\mathfrak{X}_{2}^{\text {rig }}}^{0}\right) \longrightarrow$ $\Gamma\left(\mathfrak{X}_{1}^{\text {rig }}, \mathcal{O}_{\mathfrak{X}_{1}^{\text {rig }}}^{0}\right)$. Il est immédiat de vérifier que la composée

$$
\operatorname{Hom}\left(\mathfrak{X}_{1}, \mathfrak{X}_{2}\right) \longrightarrow \operatorname{Hom}\left(\mathfrak{X}_{1}^{\text {rig }}, \mathfrak{X}_{2}^{\text {rig }}\right) \longrightarrow \operatorname{Hom}\left(\mathfrak{X}_{1}, \mathfrak{X}_{2}\right)
$$

est l'identité. De plus si $U \subset \mathfrak{X}_{i}^{r i g}$ est un ouvert affinoïde qui est une boule fermée après choix d'un système de coordonnées alors l'application

$$
\Gamma\left(\mathfrak{X}_{i}^{r i g}, \mathcal{O}_{\mathfrak{X}_{i}^{r i g}}^{0}\right)\left[\frac{1}{p}\right] \longrightarrow \Gamma\left(U, \mathcal{O}_{\mathfrak{X}_{i}^{\text {rig }}}\right)
$$


est d'image dense. On en déduit que tout morphisme $\mathfrak{X}_{1}^{\text {rig }} \longrightarrow \mathfrak{X}_{2}^{\text {rig }}$ est déterminé par la restriction du comorphisme associé à l'algèbre des fonctions holomorphes bornées. Cela implique que la composée

$$
\operatorname{Hom}\left(\mathfrak{X}_{1}^{r i g}, \mathfrak{X}_{2}^{\text {rig }}\right) \longrightarrow \operatorname{Hom}\left(\mathfrak{X}_{1}, \mathfrak{X}_{2}\right) \longrightarrow \operatorname{Hom}\left(\mathfrak{X}_{1}^{\text {rig }}, \mathfrak{X}_{2}^{\text {rig }}\right)
$$

est l'identité.

Proposition 29. Le foncteur fibre générique $\operatorname{pdiv}_{\mathcal{O}_{K}}^{f} \longrightarrow \mathcal{R}_{K}^{a}$ qui à $H$ associe $H^{\text {rig }}$ est pleinement fidèle.

Démonstration. Soient $H_{1}, H_{2} \in \operatorname{pdiv}_{\mathcal{O}_{K}}^{f}$. Il y a un diagramme

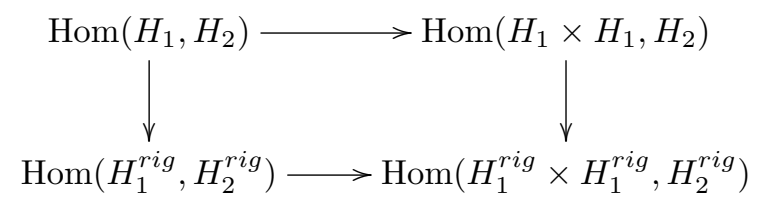

où le symbole Hom désigne ici les morphismes de $\operatorname{Spf}\left(\mathcal{O}_{K}\right)$-schémas formels et d'espaces rigides et les applications horizontales sont données par $f \longmapsto[(x, y) \mapsto f(x+y)-f(x)-f(y)]$. D'après le lemme 22 les applications verticales sont des isomorphismes. On en déduit le résultat.

Corollaire 19. Soient $H_{1}$ et $H_{2}$ deux groupes formels p-divisibles sur $\mathcal{O}_{K}$. Alors

$\operatorname{Hom}\left(H_{1}, H_{2}\right) \stackrel{\sim}{\longrightarrow}\left\{f \in \operatorname{Hom}_{\mathbb{Z}_{p}[\operatorname{Gal}(\bar{K} \mid K)]}\left(T_{p}\left(H_{1}\right), T_{p}\left(H_{2}\right)\right) \mid f \otimes 1\left(\right.\right.$ Lie H $\left._{1} \otimes C(1)\right) \subset f \otimes 1\left(\right.$ Lie H $\left.\left._{2} \otimes C(1)\right)\right\}$

où pour un groupe formel $p$-divisible $H$ sur $\mathcal{O}_{K}$, Lie $H \otimes_{K} C(1)$ est plongé dans $V_{p}(H) \otimes_{\mathbb{Q}_{p}} C$ via la transposée de l'application de Hodge-Tate de $H^{D}$ tordue par C(1).

Voici une application du théorème précédent qui fournit une généralisation du théorème de pleine fidélité de Tate ([17]).

Théorème 5.1. Supposons que $C(-1)^{G a l(\bar{K} \mid K)}=0$. Alors le foncteur fibre générique

$$
\operatorname{pdiv}_{\mathcal{O}_{K}}^{f} \longrightarrow \operatorname{pdiv}_{K}
$$

est pleinement fidèle i.e. si $H_{1}$ et $H_{2}$ sont deux groupes p-divisibles formels sur $\mathcal{O}_{K}$ alors

$$
\operatorname{Hom}\left(H_{1}, H_{2}\right) \stackrel{\sim}{\longrightarrow} \operatorname{Hom}_{\mathbb{Z}_{p}[\operatorname{Gal}(\bar{K} \mid K)]}\left(T_{p}\left(H_{1}\right), T_{p}\left(H_{2}\right)\right)
$$

Démonstration. D'après le théorème II.1.1 de [12] si $H$ est un groupe $p$-divisible sur $\mathcal{O}_{K}$ il y a une suite exacte de Hodge-Tate

$$
0 \longrightarrow \omega_{H}^{*} \otimes_{\mathcal{O}_{K}} C(1) \stackrel{\alpha_{H}^{\vee}(1)}{\longrightarrow} V_{p}(H) \otimes_{\mathbb{Q}_{p}} C \stackrel{\alpha_{H}}{\longrightarrow} \omega_{H^{D}} \otimes_{\mathcal{O}_{K}} C \longrightarrow 0 .
$$

Cette suite est une suite de $C$-espaces vectoriels munis d'une action semi-linéaire de $\operatorname{Gal}(\bar{K} \mid K)$. L'hypothèse du théorème couplée au théorème d'Ax-Sen-Tate implique que

$$
\text { Lie } H=\left(V_{p}(H) \otimes_{\mathbb{Q}_{p}} C(-1)\right)^{\operatorname{Gal}(\bar{K} \mid K)}
$$

Avec les notations de l'énoncé si $f: T_{p}\left(H_{1}\right) \longrightarrow T_{p}\left(H_{2}\right)$ est compatible à l'action de Galois on a donc

$$
\begin{aligned}
f \otimes 1\left(\operatorname{Lie} H_{1}\right) & =f \otimes 1\left(\left(V_{p}\left(H_{1}\right) \otimes C(-1)\right)^{\operatorname{Gal}(\bar{K} \mid K)}\right) \\
& \subset\left(V_{p}\left(H_{2}\right) \otimes C(-1)\right)^{\operatorname{Gal}(\bar{K} \mid K)} \\
& =\operatorname{Lie} H_{2}
\end{aligned}
$$

On peut alors appliquer le corollaire 19 . 
5.3. Filtration de Harder-Narasimhan dans la catégorie exacte des groupes de type $p$-divisible sans facteur direct additif à isogénie près. On a deux fonctions additives

$$
\text { ht, } \operatorname{dim}: \mathcal{R}_{K}^{a} \otimes \mathbb{Q} \longrightarrow \mathbb{N} \text {. }
$$

Définition 11. Si $G \in \mathcal{R}_{K}^{a} \otimes \mathbb{Q}$ est non nul on pose

$$
\mu(G)=\frac{\operatorname{dim} G}{\operatorname{ht} G} \in[0,1] .
$$

Définition 12. Un objet non-nul $G$ dans $\mathcal{R}_{K}^{a} \otimes \mathbb{Q}$ sera dit semi-stable si pour tout sous-objet non nul $G^{\prime}$ de $G, \mu\left(G^{\prime}\right) \leq \mu(G)$.

Théorème 5.2. Soit $G \in \mathcal{R}_{K}^{a} \otimes \mathbb{Q}$. Il possède alors une unique filtration dans la catégorie exacte $\mathcal{R}_{K}^{a} \otimes \mathbb{Q}$

$$
0=G_{0} \subsetneq G_{1} \subsetneq \cdots \subsetneq G_{r}=G
$$

telle que

(1) pour tout $1 \leq i \leq r, G_{i} / G_{i-1}$ soit semi-stable

(2) $\mu\left(G_{1} / G_{0}\right)>\mu\left(G_{2} / G_{1}\right)>\cdots>\mu\left(G_{r} / G_{r-1}\right)$

Démonstration. La catégorie $\mathcal{R}^{a} \otimes \mathbb{Q}$ est équivalente à celle des triplets $(W, V, f)$ où $f$ est injective. On renvoie au chapitre 10 de [11] où les filtrations de ce type d'objets sont étudiées en détail.

Le théorème précédent admet l'interprétation suivante. Supposons la valuation de $K$ discrète. Dans [11] on a démontré (cf. théorèmes 5 et 6 du chapitre 8 ) que si $H$ est un groupe $p$-divisible sur $\mathcal{O}_{K}$ il est isogène à un groupe $p$-divisible $H^{\prime}$ muni d'une filtration par des groupes $p$-divisibles

$$
0=H_{0}^{\prime} \subsetneq H_{1}^{\prime} \subsetneq \cdots \subsetneq H_{r}^{\prime}=H^{\prime}
$$

telle que

- les points de $p$-torsion des quotients, $\left(H_{i}^{\prime} / H_{i-1}^{\prime}\right)[p]$, pour $1 \leq i \leq r$, vérifient une certaine condition de semi-stabilité

- la suite $\left(\frac{\operatorname{dim} H_{i}^{\prime} / H_{i-1}^{\prime}}{\mathrm{ht} H_{i}^{\prime} / H_{i-1}^{\prime}}\right)_{1 \leq i \leq r}$ est strictement décroissante

- dans la catégorie abélienne $\operatorname{pdiv}_{\mathcal{O}_{K}} \otimes \mathbb{Q}$ la filtration précédente induit la filtration de HarderNarasimhan associée à la fonction pente $X \mapsto \frac{\operatorname{dim} X}{\mathrm{ht} X}$.

Supposons $H$ formel. D'après le théorème 20 de [11] la filtration induite

$$
0=H_{0}^{\prime r i g} \subsetneq H_{1}^{\prime r i g} \subsetneq \cdots \subsetneq H_{r}^{\prime r i g}=H^{\prime r i g}
$$

est la filtration du théorème 5.2 précédent. Le théorème précédent montre donc que même lorsque la valuation de $K$ n'est plus discrète qui à remplacer $H$ par $H^{r i g}$ et $\operatorname{pdiv}_{\mathcal{O}_{K}} \otimes \mathbb{Q}$ par $\mathcal{R}^{a} \otimes \mathbb{Q}$ on a de telles filtrations de Harder-Narasimhan, filtration qui ne provient pas forcément d'une filtration $\operatorname{dans}_{\operatorname{pdiv}} \operatorname{O}_{K} \otimes \mathbb{Q}$.

\section{Caractérisation GÉOmÉtrique des Groupes formels $p$-Divisibles}

Soit $K$ un corps valué complet comme précédemment.

Théorème 6.1. Le foncteur fibre générique

$$
\begin{aligned}
\operatorname{pdiv}_{\mathcal{O}_{K}}^{f} & \longrightarrow \mathcal{R}_{K} \\
H & \longmapsto H^{r i g}
\end{aligned}
$$

induit un isomorphisme de catégories entre la catégorie des groupes formels p-divisibles sur $\mathcal{O}_{K}$ et la catégorie des groupes analytiques rigides commutatifs $G$ tels que

- comme $K$-espace analytique rigide $G \simeq \stackrel{\circ}{B}^{d}(0,1)$ pour un entier $d$

- la multiplication par $p, \times p: G \longrightarrow G$, est un morphisme fini 
Démonstration. D'après la proposition 29 on sait déjà que le foncteur fibre générique est pleinement fidèle. Soit maintenant $G$ un groupe analytique rigide commutatif vérifiant les hypothèses du théorème. Soit

$$
A=\Gamma\left(G, \mathcal{O}_{G}^{0}\right)=\left\{f \in \Gamma\left(G, \mathcal{O}_{G}\right) \mid\|f\|_{\infty} \leq 1\right\}
$$

Puisque $G \simeq \stackrel{\circ}{\mathbb{B}}^{d}(0,1), A \simeq \mathcal{O}_{K} \llbracket x_{1}, \ldots, x_{d} \rrbracket$. Posons $H=\operatorname{Spf}(A)$. Il y a donc un isomorphisme canonique

$$
H^{r i g} \stackrel{\sim}{\longrightarrow} G .
$$

D'après le lemme 22 la loi de groupe $G \times G \longrightarrow G$ induit un morphisme $H \times H \longrightarrow H$ vérifiant les conditions d'associativité et de commutativité. L'égalité $H\left(\mathcal{O}_{K}\right)=G(K)$ fournit une section de $H \longrightarrow \operatorname{Spf}\left(\mathcal{O}_{K}\right)$ correspondant à la section neutre du $K$-groupe $G$. L'inversion $g \mapsto g^{-1}$ qui est un morphisme $G \longrightarrow G$ fournit toujours d'après le lemme 22 un morphisme $H \longrightarrow H$. On vérifie aussitôt que ces données définissent une structure de groupe formel formellement lisse sur $H$ telle que $H^{r i g} \stackrel{\sim}{\longrightarrow} G$ comme groupes analytiques rigides. Montrons que ce groupe formel est $p$-divisible.

Notons $f: G \longrightarrow G$ le morphisme multiplication par $p$ et $N$ sont degré. Soit $x \in A$. Puisque $f$ est étale fini le faisceau $f_{*} \mathcal{O}_{G}$ est un $\mathcal{O}_{G}$-module localement libre de rang $d$. Voyant $x$ comme un élément de $\Gamma\left(G, f_{*} \mathcal{O}_{G}\right)$ il définit un endomorphisme $\mathcal{O}_{G}$-linéaire « multiplication par $x \gg$ sur le faisceau d'algèbres $f_{*} \mathcal{O}_{G}$. Puisque $f_{*} \mathcal{O}_{G}$ est localement libre de rang $N$ ce morphisme est annulé par son polynôme caractéristique. On en déduit qu'il existe $a_{1}, \ldots, a_{d} \in \Gamma\left(G, \mathcal{O}_{G}\right)$ tels que

$$
x^{N}+f^{*}\left(a_{1}\right) x^{d-1}+\cdots+f^{*}\left(a_{d}\right)=0
$$

où $f^{*}: \Gamma\left(G, \mathcal{O}_{G}\right) \longrightarrow \Gamma\left(G, \mathcal{O}_{G}\right)$. Montrons que pour tout $i, a_{i} \in A=\Gamma\left(G, \mathcal{O}_{G}^{0}\right)$. Soit $U=\operatorname{Sp}(\mathrm{B})$ un ouvert affinoïde connexe de $G$ et $V=\operatorname{Sp}(C)=f^{-1}(U)$. Si $L \mid K$ une extension de degré fini le morphisme d'algèbres affinoïdes $\mathrm{B} \longrightarrow \mathrm{B} \otimes_{K} L$ est une isométrie pour les normes $\|\cdot\|_{\infty}$. On peut donc supposer qui à remplacer $G$ par $G \otimes_{K} L$ avec $L \mid K$ finie que $G\left[p^{n}\right](K)=G\left[p^{n}\right](\bar{K})$. Le morphisme $f_{\mid V}: V \longrightarrow U$ est alors un revêtement galoisien de groupe $G\left[p^{n}\right](K)$. L'égalité suivante est vérifiée dans $C[X]$

$$
X^{N}+f^{*}\left(a_{1}\right) X^{d-1}+\cdots+f^{*}\left(a_{N-1}\right) X+f^{*}\left(a_{N}\right)=\prod_{\gamma \in G\left[p^{n}\right](K)}\left(X-\gamma^{*} x\right) .
$$

Puisque $x \in C^{0}$, et donc pour tout $\gamma$ élément de $G\left[p^{n}\right](K), \gamma^{*} x \in C^{0}$, on en déduit que pour tout $i, f^{*}\left(a_{i}\right) \in C^{0}$. Le morphisme $f_{\mid V}: V \longrightarrow U$ étant fini et surjectif le morphisme d'algèbres affinoïdes $\mathrm{B} \longrightarrow C$ est une isométrie pour les normes $\|\cdot\|_{\infty}$. Donc pour tout $i, a_{i} \in \mathrm{B}^{0}$. Cela étant vrai pour tout ouvert affinoïde connexe $U$ de $G$ on en déduit que pour tout $i$

$$
a_{i} \in \lim _{U}^{\longleftarrow} \Gamma\left(U, \mathcal{O}_{G}^{0}\right)=\Gamma\left(G, \mathcal{O}_{G}^{0}\right)=A
$$

On a donc démontré que le morphisme $f^{*}: A \longrightarrow A$ est entier.

Soit $\mathfrak{m}_{A}$ l'idéal maximal de $A$. On a

$$
\begin{aligned}
\mathfrak{m}_{A} & =\{x \in A|\forall z \in G,| x(z) \mid<1\} \\
& =\{x \in A|\exists z \in G,| x(z) \mid<1\} .
\end{aligned}
$$

Le même raisonnement que précédemment montre alors que si $x \in \mathfrak{m}_{A}$ alors pour tout $i$,

$$
a_{i} \in \mathfrak{m}_{A}=\lim _{U} \Gamma\left(U, \mathcal{O}_{G}^{00}\right)
$$

où $U$ parcourt les ouverts connexes affinoïdes de $G$.

Fixons maintenant un isomorphisme $A \simeq \mathcal{O}_{K} \llbracket x_{1}, \ldots, x_{d} \rrbracket$ tel que la section neutre de $G$ soit donnée par $x_{1}=\cdots=x_{d}=0$. Via cet isomorphisme $\mathfrak{m}_{A}=\mathfrak{m}_{K}+A . x_{1}+\cdots+A . x_{d}$. Pour $1 \leq i \leq d$ soit $\left(a_{i, j}\right)_{1 \leq i \leq N} \in \mathfrak{m}_{A}^{N}$ telle que

$$
x_{i}^{N}+\sum_{j=0}^{N-1} f^{*}\left(a_{i, N-j}\right) x_{i}^{j}=0 .
$$


Soit l'idéal de $A$

$$
I=\sum_{i, j} A \cdot a_{i, j} \subset \mathfrak{m}_{A} .
$$

Pour $1 \leq i \leq d, x_{i}^{N}$ est nul dans $A / A \cdot f^{*}(I)$. Il y a donc une factorisation

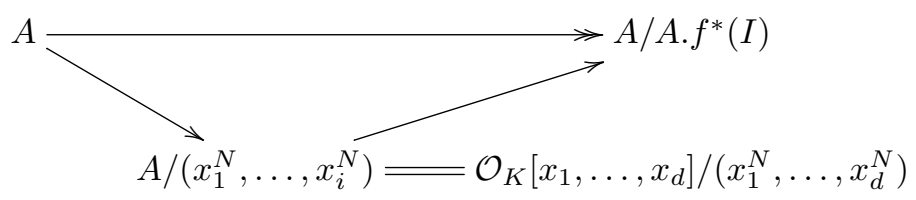

De cela on déduit que $A / A \cdot f^{*}(I)$ est un $\mathcal{O}_{K}$-module de type fini. En particulier via $A \stackrel{f^{*}}{\longrightarrow} A \rightarrow$ $A / A . f^{*}(I), A / A . f^{*}(I)$ est un $A$-module de type fini. Constatons maintenant qu'étant donné que $A . f^{*}(I)$ est un idéal de type fini de $A$ contenu dans $\mathfrak{m}_{A}, A$ est séparé complet pour la topologie $A . f^{*}(I)$-adique c'est à dire via $f^{*}: A \longrightarrow A, A$ est un $A$-module $I$-adiquement séparé complet. Le lemme de Nakayama permet de conclure que $f^{*}: A \longrightarrow A$ fait de $A$ un $A$-module de type fini.

Remarquons maintenant que si la valuation de $K$ est discrète la démonstration est terminée. En effet, le morphisme de multiplication par $p, f: G \longrightarrow G$, étant étale fini et $G$ connexe il est surjectif. De cela on déduit que $f^{*}: \Gamma\left(G, \mathcal{O}_{G}\right) \longrightarrow \Gamma\left(G, \mathcal{O}_{G}\right)$ est injectif et donc $f^{*}: A \longrightarrow A$ l'est également. L'anneau $A$ étant régulier, $f^{*}: A \longrightarrow A$ étant injectif et faisant de $A$ un $A$-module de type fini, $f^{*}: A \longrightarrow A$ est plat fini et fait de $A$ un $A$-module libre.

Cependant si la valuation de $K$ n'est pas discrète l'anneau $A$ n'est pas noethérien. Commençons par remarque qu'il suffit de montrer que pour tout entier $n \geq 1$ le morphisme fini

$$
\bar{f}^{*}: A / p^{n} A \longrightarrow A / p^{n} A
$$

fait de $A / p^{n} A$ un $A / p^{n} A$-module libre. Soit donc $n \geq 1$. Avec les notations précédentes on a déjà vu que pour $1 \leq i \leq d, x_{i}$ est nilpotent dans l'anneau $A / A . f^{*}(I)$. Soit $J=\left(p^{n}, x_{1}, \ldots, x_{d}\right) \subset A$. Puisque $I$ est un idéal de type fini contenu dans $\mathfrak{m}_{A}$ pour $k \gg 0, I^{k} \subset J$. On en déduit que pour $1 \leq i \leq d, x_{i}$ est nilpotent dans $A / A . f^{*}(J)$. Le théorème 5.8 du chapitre $\mathrm{V}$ de [18] montre alors que $A / p^{n} A$ est un $A / p^{n} A$-module libre.

Dans la même veine voici une caractérisation des groupes additifs.

Proposition 30. Soit $G$ un $K$-groupe analytique rigide de type p-divisible. Il est isomorphe à une somme de copies du groupe $\mathbb{G}_{a}^{\text {rig }}$ si et seulement si comme espace rigide analytique $G \simeq\left(\mathbb{A}^{d}\right)^{\text {rig }}$ pour un entier $d$.

Démonstration. Il s'agit de montrer que $G\left[p^{\infty}\right]=0$. On peut donc supposer $K$ algébriquement clos. D'après le théorème 3.3 si $\Lambda=T_{p}\left(G\left[p^{\infty}\right]\right)$ il existe un morphisme $f:$ Lie $G \longrightarrow \Lambda \otimes_{\mathbb{Z}_{p}} K(-1)$ ainsi qu'un morphisme de suites exactes

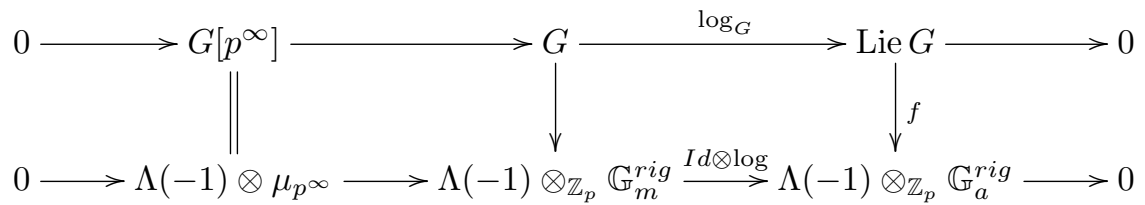

Soit $h=\mathrm{ht}(G)$. Le morphisme d'espaces analytiques rigides

$$
\left(\mathbb{A}^{d}\right)^{r i g} \simeq G \longrightarrow \Lambda(-1) \otimes_{\mathbb{Z}_{p}} \mathbb{G}_{m}^{r i g} \simeq \stackrel{\circ}{B}^{h}(0,1)
$$

est nul puisque tout fonction holomorphe bornée sur $\left(\mathbb{A}^{d}\right)^{r i g}$ est constante. On en déduit le résultat en utilisant le diagramme précédent.

7. Une Autre Classification Des $C$-Groupes anAlytiques Rigides De type $p$-Divisible

7.1. Classification des $C$-groupes analytiques rigides de type $p$-divisible connexes de dimension 1 . 
Théorème 7.1. Soit $G$ un $C$-groupe analytique rigide de type p-divisible connexe de dimension 1. Alors soit $G \simeq \mathbb{G}_{a}^{\text {rig }}$ soit il existe un groupe formel $p$-divisible de dimension 1 sur $\mathcal{O}_{C}, H$, bien déterminé à isomorphisme unique près tel que $G \simeq H^{\text {rig }}$.

Démonstration. Soit $(W, V, f)$ le triplet associé à $G$ par le théorème 3.4 où $f: W(1) \longrightarrow V_{C}$. On a donc $\operatorname{dim}_{C} W=1$. Considérons la transposée

$$
\alpha:={ }^{t} f: V^{*} \otimes_{\mathbb{Q}_{p}} C \longrightarrow W^{*}(-1) .
$$

D'après le lemme 28, $G$ est sans facteur direct additif si et seulement si $\alpha$ définit un point de l'espace projectif $\mathbb{P}\left(V_{C}\right)$. Toujours d'après le lemme 28, $G$ est de plus connexe si et seulement si $\alpha$ définit un point dans $\Omega(C)$ où $\Omega \subset \mathbb{P}(V)^{\text {rig }}$ désigne l'espace de Drinfeld. Le théorème est alors une conséquence du théorème principal du chapitre II de [12] qui implique que tout point de $\Omega$ provient par transposition de l'application de Hodge-Tate du dual d'un groupe de Lubin-Tate.

Remarque 10. Voici une approche plus géométrique à la démonstration du théorème précédent (malheureusement l'auteur n'a pas réussi à faire converger cet argument). D'après le théorème 6.1 et la proposition 30 il s'agit de montrer que comme espace analytique rigide soit $G \simeq\left(\mathbb{A}^{1}\right)^{\text {rig }}$ soit $G \simeq \stackrel{\leftrightarrow}{\mathbb{B}}(0,1)$. Soit $U$ un sous-groupe affinö̈de de $G$ tel que pour tout $n \geq 1, p^{-n} U$ soit connexe (confère lemme 14). Notons $U_{n}=p^{-n} U$. D'après la proposition 17 pour tout $n, U_{n} \simeq \mathbb{B}(0,1)$. D'après le théorème 9.7.2/2 de $[6]$ si $j: \mathbb{B}(0,1) \hookrightarrow \mathbb{B}(0,1)$ est une immersion ouverte telle que $j(0)=0$ il existe un unique $\epsilon \in\left|C^{\times}\right|, \epsilon \leq 1$ ainsi qu'une factorisation

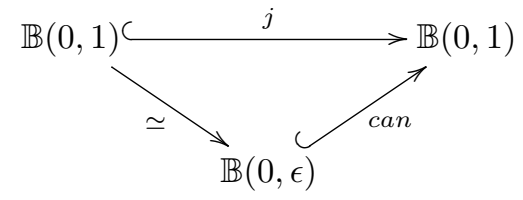

où la flèche verticale de gauche est un isomorphisme. De cela on déduit qu'il existe une suite strictement croissante d'éléments de $\left|C^{\times}\right|,\left(\epsilon_{i}\right)_{i \geq 0}$, ainsi que pour tout $i \geq 0$ un automorphisme $\varphi_{i} \in \operatorname{Aut}\left(\mathbb{B}\left(0, \epsilon_{i}\right)\right)$ tels que comme espace rigide $\bar{G}$ soit la limite inductive du diagramme

$$
\mathbb{B}\left(0, \epsilon_{0}\right) \stackrel{\text { cano }_{0}}{\longrightarrow} \mathbb{B}\left(0, \epsilon_{1}\right) \stackrel{\text { cano }_{1}}{\longrightarrow} \ldots \stackrel{\operatorname{cano}_{i-1}}{\longrightarrow} \mathbb{B}\left(0, \epsilon_{i}\right) \stackrel{\text { cano } \varphi_{i}}{\longrightarrow} \mathbb{B}\left(0, \epsilon_{i+1}\right) \stackrel{\text { cano } \varphi_{i+1}}{\longrightarrow} \ldots
$$

où « can $\gg$ désigne l'inclusion canonique d'une boule dans l'autre. Lorsque $\lim _{i \rightarrow+\infty} \epsilon_{i}=+\infty$ on peut alors montrer que $G \simeq \mathbb{A}^{1}$. Cependant lorsque $\epsilon_{\infty}=\lim _{i \rightarrow+\infty} \epsilon_{i}<+\infty$ l'auteur n'a pas réussi à montrer que $G \simeq \mathbb{B}\left(0, \epsilon_{\infty}\right)$.

\subsection{Une classification.}

Théorème 7.2. Soit $G$ un $C$-groupe analytique rigide de type p-divisible. Il existe alors

- des entiers $a, b \in \mathbb{N}$,

- des groupes formels p-divisibles de dimension $1, \mathcal{G}_{1}, \ldots, \mathcal{G}_{r} \operatorname{sur} \operatorname{Spf}\left(\mathcal{O}_{C}\right)$

- un sous-groupe p-divisible $\Gamma \subset \bigoplus_{i=1}^{r} \mathcal{G}_{i}^{\text {rig }}\left[p^{\infty}\right]$

tels que $G$ soit isomorphe au groupe

$$
G \simeq\left(\mathbb{Q}_{p} / \mathbb{Z}_{p}\right)^{a} \oplus\left(\mathbb{G}_{a}^{r i g}\right)^{b} \oplus\left(\bigoplus_{i=1}^{r} \mathcal{G}_{i}^{r i g}\right) / \Gamma .
$$

Démonstration. Utilisant les corollaire 9 et 10 on peut se ramener au cas où $G$ est connexe sans facteur direct additif. Soit alors

$$
\text { Lie } G=\bigoplus_{i=1}^{r} L_{i}
$$

une décomposition de $\operatorname{Lie} G$ en somme directe de droites. Notons pour tout $i, 1 \leq i \leq r, G_{i}$ le groupe analytique rigide de dimension 1 obtenu par image réciproque via $L_{i} \hookrightarrow \operatorname{Lie} G, G_{i}=$ 
$\log _{G}^{-1}\left(L_{i} \otimes \mathbb{G}_{a}^{r i g}\right)$

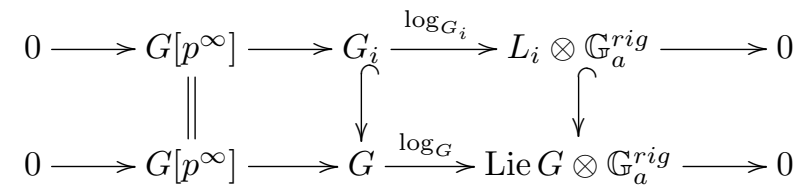

Il y a alors un morphisme étale surjectif

$$
\bigoplus_{i=1}^{r} G_{i} \longrightarrow G
$$

de noyau le groupe $p$-divisible $\Delta=\left\{\left(x_{i}\right)_{1 \leq i \leq r} \in G\left[p^{\infty}\right]^{r} \mid \sum_{i} x_{i}=0\right\}$. D'après le théorème 7.1 précédent et le corollaire 9 il existe pour tout $i$ un groupe formel $p$-divisible de dimension 1 sur $\mathcal{O}_{C}, \mathcal{G}_{i}$, ainsi qu'un groupe $p$-divisible $\Gamma_{i}$ sur $C$ tels que

$$
G_{i} \simeq \mathcal{G}_{i}^{r i g} \oplus \Gamma_{i}
$$

Identifions $G_{i}$ et $\mathcal{G}_{i}^{r i g} \oplus \Gamma_{i}$. Puisque $G$ est connexe le morphisme composé

$$
\Delta \hookrightarrow \bigoplus_{i=1}^{r} \mathcal{G}_{i}^{r i g} \oplus \Gamma_{i} \longrightarrow \bigoplus_{i=1}^{r} \Gamma_{i}
$$

est surjectif. Si $\Gamma=\operatorname{ker}\left(\Delta \longrightarrow \bigoplus_{i=1}^{r} \Gamma_{i}\right)$ alors

$$
\left(\bigoplus_{i=1}^{r} \mathcal{G}_{i}^{r i g} \oplus \Gamma_{i}\right) / \Delta=\left(\bigoplus_{i=1}^{r} \mathcal{G}_{i}^{r i g}\right) / \Gamma
$$

Remarque 11. Après qu'une version préliminaire de cet article soit apparue, Scholze et Weinstein ont démontré dans [16] qu'en fait tout groupe rigide analytique de type p-divisible sans facteur additif est la fibre générique d'un groupe p-divisible.

\section{Quasi-MORPhismes De GRoupes ANAlytiques RIGIDES DE TYPE $p$-DIVISIBLE}

\subsection{Définitions et premières propriétés.}

Définition 13. Soient $G$ et $G^{\prime}$ deux groupes analytiques rigides de type p-divisible. Un quasimorphisme entre $G$ et $G^{\prime}$ est un morphisme d'espaces rigides $f: G \longrightarrow G^{\prime}$ tel que le morphisme

$$
\begin{aligned}
G \times G & \longrightarrow G^{\prime} \\
(x, y) & \longmapsto f(x+y)-f(x)-f(y)
\end{aligned}
$$

est borné au sens où son image est contenue dans un ouvert admissible quasicompact de $G^{\prime}$ (ou de façon équivalente l'image du morphisme associé d'espaces analytiques est relativement compacte)

Tout morphisme « borné $\gg$ d'espaces rigides $G \longrightarrow G^{\prime}$, c'est à dire dont l'image est contenue dans un ouvert admissible quasicompact, est un quasi-morphisme. L'ensemble des quasi-morphismes de $G$ vers $G^{\prime}$ forme un groupe abélien via la loi de groupe de $G^{\prime}$. Les morphismes bornés forment un sous-groupe.

Définition 14. On appelle groupe des classes d'équivalence de quasi-morphismes de $G$ vers $G^{\prime}$ le groupe quotient du groupes des quasi-morphismes de $G$ vers $G^{\prime}$ par le sous-groupe des morphismes bornés. On le note $Q H o m\left(G, G^{\prime}\right)$.

Lemme 23. L'application $\operatorname{Hom}\left(G, G^{\prime}\right) \longrightarrow Q \operatorname{Hom}\left(G, G^{\prime}\right)$ est injective. 
Démonstration. Soit $f: G \longrightarrow G^{\prime}$ un morphisme de groupe qui est borné. Considérons le diagramme commutatif

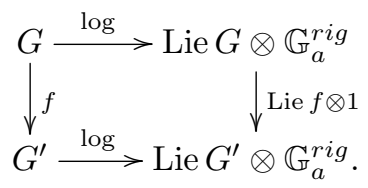

Puisque $\operatorname{Im}(f)$ est borné son image par le logarithme l'est également et donc (Lie $f \otimes 1)\left(\operatorname{Lie} G \otimes \mathbb{G}_{a}^{\text {rig }}\right)$ est borné. Cela implique que Lie $f=0$. Le morphisme $f$ se factorise donc en un morphisme $G \longrightarrow G^{\prime}\left[p^{\infty}\right]$. Via la projection $G \longrightarrow \underline{\pi}_{0}(G)$ il se factorise donc par un morphisme de groupes $p$-divisibles sur $K, \underline{\pi}_{0}(G) \longrightarrow G^{\prime}\left[p^{\infty}\right]$. Or un morphisme de groupes $p$-divisibles est borné si et seulement si son image est finie si et seulement si ce morphisme est nul. On en déduit que $f=0$.

Lemme 24. - Soient $\Gamma$ et $\Gamma^{\prime}$ deux groupes p-divisibles. Alors

$$
Q \operatorname{Hom}\left(\Gamma, \Gamma^{\prime}\right)=\operatorname{Hom}\left(\Gamma, \Gamma^{\prime}\right) \otimes \mathbb{Q}_{p}
$$

- Soient $V$ et $V^{\prime}$ deux $K$-espaces vectoriels de dimension finie

$$
Q H o m\left(V \otimes \mathbb{G}_{a}^{r i g}, V^{\prime} \otimes \mathbb{G}_{a}^{r i g}\right)=\operatorname{Hom}\left(V \otimes \mathbb{G}_{a}^{r i g}, V^{\prime} \otimes \mathbb{G}_{a}^{r i g}\right)=\operatorname{Hom}\left(V, V^{\prime}\right)
$$

Démonstration. Si $f: \Gamma \longrightarrow \Gamma^{\prime}$, dire que $(x, y) \mapsto f(x+y)-f(x)-f(y)$ est bornée est équivalent à dire qu'il existe $n \geq 1$ tel que $\forall x, y \in \Gamma, p^{n}(f(x+y)-f(x)-f(y))=0$. Cela est encore équivalent à dire qu'il existe $n \geq 1$ tel que la composée $\Gamma \stackrel{f}{\longrightarrow} \Gamma^{\prime} \longrightarrow \Gamma^{\prime} / \Gamma^{\prime}\left[p^{n}\right]$ soit un morphisme de groupes $p$-divisibles. De plus deux tels quasi-morphismes sont équivalents si et seulement si pour $n \gg 0$ les deux morphismes associés $\Gamma \longrightarrow \Gamma^{\prime} / \Gamma^{\prime}\left[p^{n}\right]$ sont égaux. On a donc

$$
Q \operatorname{Hom}\left(\Gamma, \Gamma^{\prime}\right)=\underset{n \geq 1}{\lim _{n \geq 1}} \operatorname{Hom}\left(\Gamma, \Gamma^{\prime} / \Gamma^{\prime}\left[p^{n}\right]\right)=\operatorname{Hom}\left(\Gamma, \Gamma^{\prime}\right) \otimes \mathbb{Q}_{p}
$$

La seconde assertion résulte de ce que toute fonction holomorphe bornée sur un espace affine est constante.

Lemme 25. Soient $G, G^{\prime}$ et $G^{\prime \prime}$ des groupes de type p-divisible. Soient $f: G \longrightarrow G^{\prime}$ et $g: G^{\prime} \longrightarrow$ $G^{\prime \prime}$ deux quasi-morphismes. Alors $g \circ f: G \longrightarrow G^{\prime \prime}$ est un quasi-morphisme. Cela définit une application bilinéaire

$$
Q \operatorname{Hom}\left(G, G^{\prime}\right) \times Q \operatorname{Hom}\left(G^{\prime}, G^{\prime \prime}\right) \longrightarrow Q \operatorname{Hom}\left(G, G^{\prime \prime}\right)
$$

Lemme 26. Soient $G, G^{\prime}, G^{\prime \prime}$ trois groupes analytiques rigides de type p-divisible. Alors

$$
\begin{gathered}
Q \operatorname{Hom}\left(G \times G^{\prime}, G^{\prime \prime}\right) \stackrel{\sim}{\longrightarrow} \operatorname{Hom}\left(G, G^{\prime \prime}\right) \oplus Q \operatorname{Hom}\left(G^{\prime}, G^{\prime \prime}\right) \\
Q \operatorname{Hom}\left(G, G^{\prime} \times G^{\prime \prime}\right) \stackrel{\sim}{\longrightarrow} Q \operatorname{Hom}\left(G, G^{\prime}\right) \oplus Q \operatorname{Hom}\left(G, G^{\prime \prime}\right)
\end{gathered}
$$

Résumons les résultats précédents dans la proposition suivante.

Proposition 31. La catégorie des groupes analytiques rigides de type p-divisible munie des classes d'équivalence de quasi-morphismes est une catégorie additive. Le foncteur canonique de la catégorie des groupes de type $p$-divisible vers cette catégorie est fidèle. Si $\mathcal{Q R}$ désigne cette catégorie les foncteurs composés

$$
\operatorname{pdiv}_{K} \longrightarrow \mathcal{R} \otimes \mathbb{Q} \longrightarrow \mathcal{Q R}
$$

et

$$
\begin{aligned}
\operatorname{Vect}_{K} & \longrightarrow \mathcal{R} \otimes \mathbb{Q} \longrightarrow \mathcal{Q R} \\
W & \longmapsto W \otimes \mathbb{G}_{a}^{\text {rig }}
\end{aligned}
$$

sont pleinement fidèles. 
8.2. Lien avec les quasi-logarithmes d'un groupe formel. Soit maintenant $\mathcal{G}=\operatorname{Spf}(R)$ un groupe formel $p$-divisible sur $\operatorname{Spf}\left(\mathcal{O}_{K}\right)$ et $\mathcal{G}^{\text {rig }}$ le groupe analytique rigide de type $p$-divisible associé. Notons $\Delta: R \longrightarrow R \hat{\otimes} R$ la comultiplication. Notons $\widehat{R\left[\frac{1}{p}\right]}$ le complété de $R\left[\frac{1}{p}\right]$ relativement à son idéal d'augmentation (i.e. $\operatorname{Spf}\left(\widehat{R\left[\frac{1}{p}\right]}\right)$ est le groupe formel fibre générique de $\left.\mathcal{G}\right)$. Rappelons qu'un quasi-logarithme sur $\mathcal{G}$ est un élément $f \in \widehat{R\left[\frac{1}{p}\right]}$ vérifiant

(1) $f$ est dans l'idéal d'augmentation de $\widehat{R\left[\frac{1}{p}\right]}$

(2) $\Delta(f)-f \otimes 1-1 \otimes f \in R\left[\frac{1}{p}\right]$

(3) $d f \in \widehat{\Omega}_{R / \mathcal{O}_{K}}^{1}\left[\frac{1}{p}\right] \subset \widehat{\Omega}^{1} \widehat{R\left[\frac{1}{p}\right] / K}$

Les quasi-logarithmes forment un groupe qui contient le sous-groupe $R\left[\frac{1}{p}\right]$, le quotient est appelé les groupes des classes d'équivalences de quasi-logarithmes.

Proposition 32. Le groupe des quasi-morphismes de $\mathcal{G}^{\text {rig }}$ vers $\mathbb{G}_{a}^{\text {rig }}$ s'identifie au groupe des quasi-logarithmes de $\mathcal{G}$. Cela induit un isomorphisme entre le groupe des classes d'équivalences de quasi-logarithmes de $\mathcal{G}$ et $Q \operatorname{Hom}\left(\mathcal{G}^{\text {rig }}, \mathbb{G}_{a}^{\text {rig }}\right)$.

Démonstration. Fixons un isomorphisme $R \simeq \mathcal{O}_{K} \llbracket x_{1}, \ldots, x_{d} \rrbracket$ qui induit $\widehat{R\left[\frac{1}{p}\right]} \simeq K \llbracket x_{1}, \ldots, x_{d} \rrbracket$ et $\operatorname{Spf}(R)^{r i g} \simeq \stackrel{\circ}{B}^{d}(0,1)$. Alors les fonctions rigides sur $\operatorname{Spf}(R)^{r i g}$ sont les $\sum_{\alpha \in \mathbb{N}^{d}} a_{\alpha} x_{1}^{\alpha_{1}} \ldots x_{d}^{\alpha_{d}} \in$ $\widehat{R\left[\frac{1}{p}\right]}$ telles que

$$
\forall \rho<1, \quad \lim _{|\alpha| \rightarrow+\infty}\left|a_{\alpha}\right| \rho^{|\alpha|}=0
$$

Les fonctions rigides bornées sont les éléments de $R\left[\frac{1}{p}\right]$.

Soit maintenant $f$ un quasi-logarithme. La condition (3) dans la définition d'un quasi-logarithme est équivalente à dire que $\forall i, \frac{\partial f}{\partial x_{i}} \in R\left[\frac{1}{p}\right]$. On vérifie facilement que cela implique que $f$ est convergente sur la boule ouverte rigide et définit une fonction $f^{r i g}: \mathcal{G}^{\text {rig }} \longrightarrow \mathbb{G}_{a}^{r i g}$. Les conditions (1) et (2) impliquent alors que $f^{\text {rig }}$ est un quasi-morphisme.

Réciproquement, soit $f$ un quasi-morphisme. Vu comme élément de $\widehat{R\left[\frac{1}{p}\right]}$ il est clair qu'il vérifie les conditions (1) et (2) de la définition d'un quasi-logarithme. Reste la condition (3). Soit $\left(\omega_{1}, \ldots, \omega_{d}\right)$ une base des formes différentielles invariantes sur $\mathcal{G}$. On a donc

$$
\widehat{\Omega}_{R / \mathcal{O}_{K}}^{1}=\bigoplus_{i=1}^{d} R \cdot \omega_{i}
$$

De plus $\left(\omega_{i}\right)_{1 \leq i \leq d}$ définit une base $\left(\omega_{i}^{\text {rig }}\right)_{1 \leq i \leq d}$ des formes invariantes sur $\mathcal{G}^{\text {rig }}$ et

$$
\Gamma\left(\mathcal{G}^{r i g}, \Omega_{\mathcal{G}^{r i g} / K}^{1}\right)=\bigoplus_{i=1}^{d} \Gamma\left(\mathcal{G}^{r i g}, \mathcal{O}_{\mathcal{G}^{r i g}}\right) \cdot \omega_{i}^{\text {rig }}
$$

Donc, $\widehat{\Omega}_{R / \mathcal{O}_{K}}^{1}\left[\frac{1}{p}\right]$ est exactement l'ensemble des formes différentielles qui s'écrivent comme combinaisons linéaires de formes invariantes à coefficients des fonctions rigides bornées. Soit

$$
f^{*} d T=\sum_{i} \lambda_{i} \omega_{i}^{r i g} \in \Gamma\left(\mathcal{G}^{r i g}, \Omega_{\mathcal{G}^{r i g} / K}\right)
$$

pour des fonctions $\lambda_{i} \in \Gamma\left(\mathcal{G}^{\text {rig }}, \mathcal{O}_{\mathcal{G}^{\text {rig }}}\right)$. Si $m: \mathcal{G}^{\text {rig }} \times \mathcal{G}^{\text {rig }} \longrightarrow \mathcal{G}^{\text {rig }}$ désigne la loi de groupe et pour $i=1,2, \operatorname{pr}_{i}: \mathcal{G}^{\text {rig }} \times \mathcal{G}^{\text {rig }} \longrightarrow \mathcal{G}^{\text {rig }}$ les deux projections, on a

$$
m^{*} f^{*} d T=\sum_{i}\left(\lambda_{i} \circ m\right) m^{*} \omega_{i}^{r i g}=\sum_{i} \lambda_{i} \circ m\left(\operatorname{pr}_{1}^{*} \omega_{i}^{r i g}+\mathrm{pr}_{2}^{*} \omega_{i}^{r i g}\right)
$$

Donc

$$
\begin{aligned}
\left(f \circ m-f \circ \mathrm{pr}_{1}-f \circ \mathrm{pr}_{2}\right)^{*} d T & =m^{*} f^{*} d T-\mathrm{pr}_{1}^{*} f^{*} d T-\mathrm{pr}_{2}^{*} f^{*} d T \\
& =\sum_{i}\left[\left(\lambda_{i} \circ m-\lambda_{i} \circ \mathrm{pr}_{1}\right) \cdot \operatorname{pr}_{1}^{*} \omega_{i}^{r i g}+\left(\lambda_{i} \circ m-\lambda_{i} \circ \operatorname{pr}_{2}\right) \cdot \mathrm{pr}_{2}^{*} \omega_{i}^{r i g}\right]
\end{aligned}
$$


Mais la fonction $\varphi=f \circ m-f \circ \operatorname{pr}_{1}-f \circ \mathrm{pr}_{2}$ sur $\mathcal{G}^{\text {rig }} \times \mathcal{G}^{\text {rig }}$ étant bornée par hypothèse, $\varphi \in(R \hat{\otimes} R)\left[\frac{1}{p}\right]$ et donc $\varphi^{*} d T \in \widehat{\Omega}_{R \hat{\otimes} R / \mathcal{O}_{K}}^{1}\left[\frac{1}{p}\right]=\operatorname{pr}_{1}^{*} \widehat{\Omega}_{R / \mathcal{O}_{K}}^{1}\left[\frac{1}{p}\right] \oplus \operatorname{pr}_{2}^{*} \widehat{\Omega}_{R / \mathcal{O}_{K}}^{1}\left[\frac{1}{p}\right]$. De cela on déduit que pour tout $i$, la fonction $\lambda_{i} \circ m-\lambda_{i} \circ \mathrm{pr}_{1}:(x, y) \mapsto \lambda_{i}(x+y)-\lambda_{i}(x)$ est bornée et que donc $\lambda_{i}$ est bornée, ce qui termine la démonstration.

Corollaire 20. Si $\mathcal{G}$ est un groupe formel p-divisible sur $\operatorname{Spf}\left(\mathcal{O}_{K}\right)$ l'algèbre de Lie de l'extension vectorielle universelle de $\mathcal{G}^{D}$ munie de sa filtration de Hodge (la partie vectorielle) s'identifie à

$$
\operatorname{Hom}\left(\mathcal{G}^{\text {rig }}, \mathbb{G}_{a}^{\text {rig }}\right) \subset Q H o m\left(\mathcal{G}^{\text {rig }}, \mathbb{G}_{a}^{\text {rig }}\right)
$$

\section{RÉFÉRENCES}

[1] Revêtements étales et groupe fondamental (SGA 1). Documents Mathématiques (Paris) [Mathematical Documents (Paris)], 3. Société Mathématique de France, Paris, 2003. Séminaire de géométrie algébrique du Bois Marie 1960-61. [Algebraic Geometry Seminar of Bois Marie 1960-61], Directed by A. Grothendieck, With two papers by M. Raynaud, Updated and annotated reprint of the 1971 original [Lecture Notes in Math., 224, Springer, Berlin; MR0354651 (50 \#7129)].

[2] James Ax. Zeros of polynomials over local fields-The Galois action. J. Algebra, 15 :417-428, 1970.

[3] V. Berkovich. Spectral theory and analytic geometry over non-Archimedean fields, volume 33 of Mathematical Surveys and Monographs. American Mathematical Society, Providence, 1990.

[4] V. Berkovich. Étale cohomology for non-archimedean analytic spaces. Inst. Hautes Études Sci. Publ. Math., $78: 5-161,1993$

[5] V. Berkovich. Vanishing cycles for formal schemes. Invent. Math., 115(3) :539-571, 1994.

[6] S. Bosch, U. Güntzer, and R. Remmert. Non-Archimedean analysis, volume 261 of Grundlehren der Mathematischen Wissenschaften. Springer-Verlag, Berlin.

[7] S. Bosch and W. Lütkebohmert. Formal and rigid geometry. I. Rigid spaces. Math. Ann., 295(2) :291-317, 1993.

[8] S. Bosch, W. Lütkebohmert, and M. Raynaud. Formal and rigid geometry. III. The relative maximum principle. Math. Ann., 302(1) :1-29, 1995

[9] P. Colmez. Espaces de Banach de dimension finie. J. Inst. Math. Jussieu, 1(3) :331-439, 2002.

[10] A. J. de Jong. Étale fundamental groups of non-Archimedean analytic spaces. Compositio Math., 97(1-2) :89118, 1995. Special issue in honour of Frans Oort.

[11] L. Fargues. Théorie de la réduction pour les groupes p-divisibles. $h t t p: / / w w w . ~ m a t h . u-p s u d$. fr/ fargues/ Prepublications. html.

[12] L. Fargues. L'isomorphisme entre les tours de Lubin-Tate et de Drinfeld et applications cohomologiques. In L'isomorphisme entre les tours de Lubin-Tate et de Drinfeld, Progress in math., 262, pages 1-325. Birkhäuser, 2008.

[13] Laurent Fargues. Les espaces de Banach-Colmez et leurs filtrations. En préparation.

[14] P. Gabriel. Groupes formels. In Schémas en Groupes (Sém. Géométrie Algébrique, Inst. Hautes Études Sci., 1963/64), Fasc. 2b, Exposé 7b, pages 66-152+3. Inst. Hautes Études Sci., Paris, 1965.

[15] W. Messing. The crystals associated to Barsotti-Tate groups : with applications to abelian schemes. Lecture Notes in Mathematics, 264, 1972.

[16] Peter Scholze and Jared Weinstein. Moduli of p-divisible groups. Camb. J. Math., 1(2) :145-237, 2013.

[17] J. T. Tate. p-divisible groups.. In Proc. Conf. Local Fields (Driebergen, 1966), pages 158-183. Springer, Berlin, 1967.

[18] Thomas Zink. Cartiertheorie kommutativer formaler Gruppen, volume 68 of Teubner-Texte zur Mathematik [Teubner Texts in Mathematics]. BSB B. G. Teubner Verlagsgesellschaft, Leipzig, 1984. With English, French and Russian summaries.

CNRS - Institut de Mathématiques de Jussieu

Email address: laurent.fargues@imj-prg.fr 